\title{
Yukawa textures from singular spectral data
}

\section{Mohsen Karkheiran}

Center for Theoretical Physics of the Universe, Institute for Basic Science (IBS), 55 Expo-ro, Daejeon, 34051, South Korea

E-mail: mohsenkar@ibs.re.kr

ABSTRACT: The Yukawa textures of effective heterotic models are studied by using singular spectral data. One advantage of this approach is that it is possible to dissect the cohomologies of the bundles into smaller parts and identify the pieces that contain the zero modes, which can potentially have non-zero Yukawa couplings. Another advantage is the manifest relationship between the Yukawa textures in heterotic models and local F-theory models in terms of fields living in bulk or localized inside the 7-branes. We only work with Weierstrass elliptically fibered Calabi-Yau manifolds here. The idea for generalizing this approach to every elliptically fibered Calabi-Yau with rational sections is given at the end of this paper.

Keywords: Superstrings and Heterotic Strings, F-Theory, String Duality, Superstring Vacua

ArXiv EPrint: 2107.07830 


\section{Contents}

1 Introduction $\quad 2$

2 General analysis and summary of results 3

2.1 Fourier-Mukai transforms and main idea 5

$\begin{array}{lll}2.2 & \text { Degree two spectral cover } & 11\end{array}$

2.3 Caveats 12

3 Spectral covers without vertical components 13

$\begin{array}{lll}3.1 & E_{6} \text { GUT models } & 13\end{array}$

$\begin{array}{lll}3.1 .1 & \text { Smooth spectral cover } & 14\end{array}$

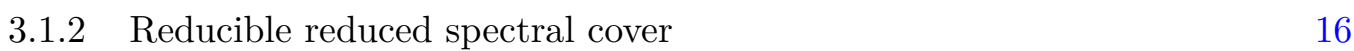

$\begin{array}{lll}3.2 & \mathrm{SO}(10) \text { GUT models } & 19\end{array}$

$\begin{array}{lll}3.2 .1 & \text { Smooth spectral cover } & 19\end{array}$

3.2.2 Reducible reduced spectral covers 22

3.2.3 Spectral covers with non-reduced components 26

$3.3 \mathrm{SU}(5)$ model 30

$\begin{array}{lll}3.3 .1 & \text { Smooth spectral cover } & 31\end{array}$

$\begin{array}{lll}\text { 3.3.2 } & \text { Reducible reduced spectral covers } & 32\end{array}$

3.3.3 Spectral cover with non-reduced components 37

4 Vertical component $\quad 42$

4.1 General results for Yukawa couplings 44

4.2 Bundles with degenerate spectral covers 45

5 Examples $\quad 48$

$5.1 E_{6}$ model 48

$\begin{array}{lll}5.2 & \mathrm{SU}(5) \text { model } & 53\end{array}$

6 Elliptic fibrations without holomorphic section $\quad 57$

$\begin{array}{lll}7 & \text { Conclusion } & 59\end{array}$

A Useful formulas/terminologies $\quad 59$ 


\section{Introduction}

One of the main goals of string phenomenology is to derive the standard model of particle physics in the low energy limit. Compactifying the heterotic string over Calabi-Yau manifolds from ten to four dimensions seems to be a promising approach [1]. In such models, one compactifies the Heterotic $E_{8} \times E_{8}$ models over a Calabi-Yau threefold and turns on a background value for the gauge fields living on the Calabi-Yau. The effective theory will be an $\mathcal{N}=1$ super-GUT model in four dimensions with gauge groups $E_{6}, \mathrm{SO}(10)$, and $\mathrm{SU}(5)[2,3]$. These models can be further broken down to MSSM. For example, see [4-23].

One of the significant challenges is computing the Yukawa couplings of the effective theory. The exact value of these couplings is tough to compute in heterotic string models. The reason is that one needs both to normalize the kinetic term of the four-dimensional action and, at the same time, know the value of the holomorphic coupling in the superpotential. This requires detailed knowledge of the Calabi-Yau metric and the solutions of the Hermitian Yang-Mills equations on the Calabi-Yau. There are many numeric studies in this direction [24-38], but we only focus on the Yukawa textures in this paper.

Generally, the Yukawa couplings appear from a Chern-Simons term in the tendimensional action [39, 40]

$$
\int_{X} \Omega \wedge \operatorname{Tr}(A \wedge A \wedge A)
$$

where $X$ is the Calabi-Yau manifold, $\Omega$ is the holomorphic top form of $X$, and $A$ is the background gauge field living inside $X$. The fluctuations of $A$ around this background value appear as four-dimensional chiral multiplets charged under the effective gauge group. Therefore these chiral multiplets are identified with the zero modes of the Dirac operator in $X$, which can be identified with the elements of the cohomologies of the vector bundle $V$ associated with the gauge field $A$, i.e., $H^{*}(V), H^{*}\left(\Lambda^{k} V\right), H^{*}\left(V^{*} \otimes V\right)$, and so on. There are several techniques to get at least Yukawa textures. One way is to reduce the problem into an algebraic one by representing the cohomology groups' elements by polynomials [41-46]. Also ,in some cases, such as monad bundles or line bundle models, where the bundle is constructed by restricting line bundles in an ambient space, it is possible to use differential geometric approaches to compute the Yukawa couplings [47, 48].

The main goal of this paper is to facilitate the computations. We will introduce new ways to compute the Yukawa textures, and we believe it can be further generalized to compute the Yukawa couplings. The secondary goal of this paper is to show the relationship between the Yukawa couplings in effective heterotic theory and the ones that appear in F-theory models. ${ }^{1}$ This is interesting because there are always intersecting branes in other string theory models, and there are fields that live over branes and the brane intersections. In such models, the appearance of Yukawa couplings in the effective theory is at least more intuitive. However, there are no branes in perturbative heterotic models, and naively, there is no connection between the Yukawa couplings in intersecting brane models and the Yukawa couplings in heterotic models. The approach that we choose to compute the

\footnotetext{
${ }^{1}$ For Yukawa couplings in F-theory and intersection brane model, generally look at [53, 54] and references inside them. For T-brane related Yukawa also look at [49, 50, 52].
} 
Yukawa couplings makes this connection explicit. We hope we can use this to study the vanishing theorems [55] and unification of Yukawa couplings [56] in future works.

More precisely, we consider $E_{8} \times E_{8}$ heterotic compactified on an elliptically fibered Calabi-Yau threefold. This is not too restrictive since many of the Calabi-Yau threefolds constructed so far are elliptically fibered [57,58]. Over such manifolds, one can study a holomorphic, stable, degree zero bundle by spectral data $(\mathcal{S}, \mathcal{L})$ [59-61], where $\mathcal{S}$ is a finite cover of the base of the elliptic fibration and $\mathcal{L}$ is a sheaf over that where its Hilbert polynomial is the same as the Hilbert polynomial of a line bundle over $\mathcal{S}$. One can use this description of bundles to study the spectrum and couplings of the effective theory [62-73].

In $[74,75]$, some selection rules have been found using spectral data and derived pushforwards. Also, in [76, 77], the Yukawa couplings in Heterotic and dual F-theory were studied. All of these works, though very impressive, were restricted to smooth spectral covers. This assumption is somewhat too restrictive. For example, the vector bundles in Heterotic (0,2)-GLSM models which, admit elliptic fibration usually, have singular spectral cover [79-81]. The main novelty of this paper is finding a way to study the Yukawa for singular spectral covers using Pontjagin products [82]. So for obvious reasons, this approach is more general.

In this paper, we focused only on elliptically fibered Calabi-Yau manifolds, which are presented by smooth Weierstrass models since the Fourier-Mukai transformation (with the kernel being Poincare sheaf [82]) works best in this situation. Nevertheless, at the end of this paper, we explain how one can generalize this approach over any elliptically fibered threefold, and hence, one can generalize the strategy we presented here to compute Yukawa couplings (textures) for most of the Heterotic models. We should also mention that one should be careful about the stability of the bundles. When the spectral cover is singular, it is not guaranteed that the corresponding bundle is stable, and hence the effective theory is supersymmetric. We explain this issue in the next section.

This paper is organized as follows. Section 2 summarizes the results, reviews the setup, and proves useful identities used extensively in later sections. Then, we explain how to compute the Pontrjagin product for a simple degree two spectral covers. Section 3 and 4 contain the main body of calculations. In section 3, we focus only on the spectral data without vertical components. We also comment on the dual F-theory models. However, as mentioned before, our purpose is not to study the F-theory dual in detail. In section 4, we consider the effect of adding a smooth vertical component. Section 5 gives toy examples for $E_{6}$ and $\mathrm{SU}(5)$ models with detailed calculations. Section 6 will discuss how one can extend these techniques to Calabi-Yau manifolds without holomorphic sections. We also collect some useful terminologies and calculations in the appendix.

\section{General analysis and summary of results}

To illustrate the idea consider $E_{6}$ models. There are chiral and anti-chiral fields in representations $\mathbf{2 7}$ and $\overline{\mathbf{2 7}}$ (for a detailed explanation, see [23] and references there). So there are 27-27-27, $\overline{\mathbf{2 7}} \overline{\mathbf{2 7}} \overline{\mathbf{2 7}}$, and 1-27-27 couplings. How they can be computed in terms of cohomologies? In principle one can use the cup product between the cohomologies to 
produce cohomologies of other bundles [44, 45]. For example for a $\mathrm{SU}(3)$ bundle

$$
H^{1}(V) \otimes H^{1}(V) \otimes H^{1}(V) \longrightarrow H^{3}\left(\Lambda^{3} V\right) \simeq H^{3}\left(\mathcal{O}_{X}\right) \simeq \mathbb{X}
$$

Elements of $H^{1}(V)$ correspond to the $\mathbf{2 7}$ chiral multiplets. If certain elements of $H^{1}(V)$ are in the image of the above homomorphism between cohomology groups, one can say the corresponding fluctuations of the gauge field can be "multiplied" to form a $(0,3)$ form which can be contracted with $\Omega$ in the Chern-Simons action (1.1) to give a non-zero Yukawa coupling in four dimensions. Similarly, the non-zero homomorphism

$$
H^{1}\left(V^{*}\right) \otimes H^{1}\left(V^{*}\right) \otimes H^{1}\left(V^{*}\right) \longrightarrow H^{3}\left(\Lambda^{3} V^{*}\right) \simeq \mathbb{C},
$$

corresponds to non-zero $\overline{\mathbf{2 7}} \overline{\mathbf{2 7}} \overline{\mathbf{2}} \overline{\mathbf{7}}$, and

$$
\operatorname{Ext}^{1}\left(\mathcal{O}_{X}, V\right) \otimes \operatorname{Ext}^{1}(V, V) \otimes \operatorname{Ext}^{1}\left(V, \mathcal{O}_{X}\right) \longrightarrow \operatorname{Ext}^{3}\left(\mathcal{O}_{X}, \mathcal{O}_{X}\right) \simeq \mathbb{C},
$$

corresponds to 1-27-27. For future reference, let us mention the couplings for the $\mathrm{SO}(10)$, and SU(5) super-GUT models,

$$
\begin{aligned}
H^{1}\left(V^{*}\right) \otimes H^{1}\left(V^{*}\right) \otimes H^{1}\left(\Lambda^{2} V^{*}\right) & \longrightarrow \mathbb{C}, \mathbf{1 6 - 1 6 - 1 0}, \mathrm{SU}(4), \\
H^{1}\left(V^{*}\right) \otimes H^{1}\left(V^{*}\right) \otimes H^{1}\left(\Lambda^{3} V^{*}\right) & \longrightarrow \mathbb{C}, \mathbf{1 0 - 1 0 - 5}, \mathrm{SU}(5), \\
H^{1}\left(V^{*}\right) \otimes H^{1}\left(\Lambda^{2} V^{*}\right) \otimes H^{1}\left(\Lambda^{2} V^{*}\right) & \longrightarrow \mathbb{C}, \mathbf{1 0 -} \overline{\mathbf{5}}-\overline{\mathbf{5}}, \mathrm{SU}(5) .
\end{aligned}
$$

Generally, to compute the Yukawa couplings, these cohomologies can be represented by polynomials. Then it can be checked directly whether these polynomials can "contract" to form an element of $H^{3}\left(\mathcal{O}_{X}\right)=H^{0}\left(\mathcal{O}_{X}\right)$ [44, 45]. This type of calculation has been used recently to carefully study the Yukawa couplings in the heterotic line bundle models [83], which is already very complicated, needless to mention how tough it can be for more general bundles.

As mentioned before, the primary goal of this paper is to give another approach to compute the Yukawa couplings. We do not claim that this approach makes the calculations much easier. However, at least, we can dissect the procedure and give smaller and simpler pieces that the chiral multiplets with potential non-zero Yukawa couplings can live there. Namely, instead of computing the cohomology of the bundle, which can be very messy, we give cohomologies of simpler sheaves like line bundles living on a curve or surface, such that the elements of these groups can correspond to chiral fields that have non-zero Yukawa couplings. However, the misery is preserved. One needs to check many "coboundary maps" to see whether the elements of these groups contribute to the cohomologies of $V$ and the associated bundles. Nevertheless, as mentioned before, at least the procedure is broken into smaller and simpler pieces, and the connection to the intersecting brane models becomes transparent.

The setup that we are mainly going to use is $(X, V)$. Where $X$ is a Weierstrass elliptically fibered Calabi-Yau threefold. In other words, it is Calabi-Yau embedded in a $\mathbb{P}^{231}$ fibration over a surface (denoted by $\left.B\right) \pi: \mathcal{A} \longrightarrow B$ with an algebraic equation

$$
Y^{2}+X^{3}+F X Z^{4}+G Z^{6}=0
$$




\begin{tabular}{|c|c|c|}
\hline Spectral Cover & Zero Modes & Possible Yukawa Couplings \\
\hline$S_{3}=a_{3} Y+a_{2} X Z+a_{0} Z^{3}$ & $\mathbf{2 7}$ on $a_{3}=0$ & No Yukawa couplings \\
\hline$S_{3}=Z\left(a_{2} X+a_{0} Z^{2}\right)$ & $\begin{array}{c}\mathbf{2 7} \text { Bulk } \\
\mathbf{2 7} a_{2}=0\end{array}$ & $\mathbf{2 7 - 2 7 - 2 7}$ on $a_{2}=0$ \\
\hline
\end{tabular}

Table 1. $E_{6}$ models Yukawa couplings.

where $(X, Y, Z)$ are coordinates of $\mathbb{P}^{231}$ with degrees $(2,3,1)$. The elliptic fibration $\pi$ : $X \longrightarrow B$ has a section denoted by $\sigma$

$$
i_{\sigma}: \sigma \hookrightarrow X
$$

such that $\pi \circ i_{\sigma}=i d_{B}$. The vector bundle $V$ is supposed to be a stable degree zero holomorphic bundle over $X$. Such kind of bundles can be constructed by spectral data introduced by Friedman, Morgan, and Witten in $[59,60] .{ }^{2}$ Spectral data is given by a doublet $\left(\mathcal{S}_{n}, \mathcal{L}_{n}\right) . \mathcal{S}_{n} \subset X$ is a finite cover of the base manifold $B$ with degree $n$ which is embedded inside $X$, and $\mathcal{L}_{n}$ is line bundle on $\mathcal{S}_{n}{ }^{3}$. We will use these spectral data to compute the Yukawa couplings.

On the other hand, local F-theory models can be defined in terms of intersection 7branes, and Higgs bundles living over these branes [49, 87]. The Higgs bundles are defined by a doublet $(E, \Phi)$ where $E$ is "flux" inside the 7-brane and $\Phi$ is 2 -form with values in $a d(E)$. The Higgs bundle can also be defined by spectral data $\left(\overline{\mathcal{S}}_{n}, \overline{\mathcal{L}}_{n}\right)$. The spectral cover (embedded in the total space of the canonical bundle) is defined as the characteristic polynomial of $\Phi$, and is a finite cover of the 7-brane. $\overline{\mathcal{L}}_{n}$ is a line bundle over the spectral cover. These two spectral covers in heterotic string theory and local F-theory are closely related [86, 89]. Therefore with our approach, the Yukawa couplings in local F-theory models can also be calculated (see figure 1). We are not going to study this side completely, but we will comment on it.

When there are vertical components, in addition to the Yukawa couplings that can emerge from the other components, there are possible extra contributions to the Yukawa couplings. However, for the SU(5) models, there are no new 10-5-5 couplings from the vertical components. We summarized the results in the tables.

\subsection{Fourier-Mukai transforms and main idea}

We start with a brief review of Fourier-Mukai transforms and spectral cover construction of stable degree zero holomorphic vector bundles. For more information, see [82]. FourierMukai is a functor which is an auto equivalence of derived category of coherent sheaves on X,

$$
\Phi: D^{b}(X) \longrightarrow D^{b}(X) .
$$

\footnotetext{
${ }^{2}$ When the spectral cover is reducible it is not guaranteed that the corresponding bundle is stable, and it must be checked case by case.

${ }^{3} n$ is the rank of $V$.
} 


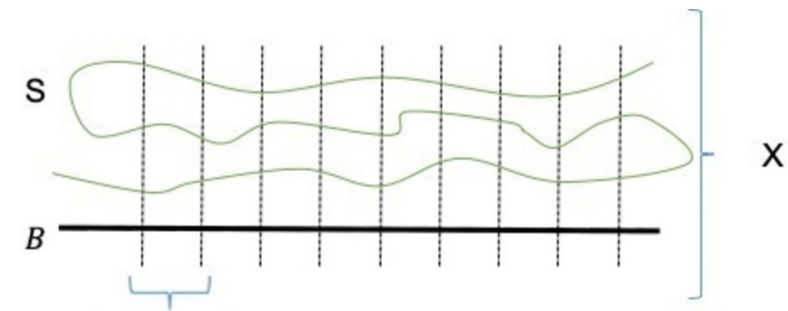

Fibers of

$X$

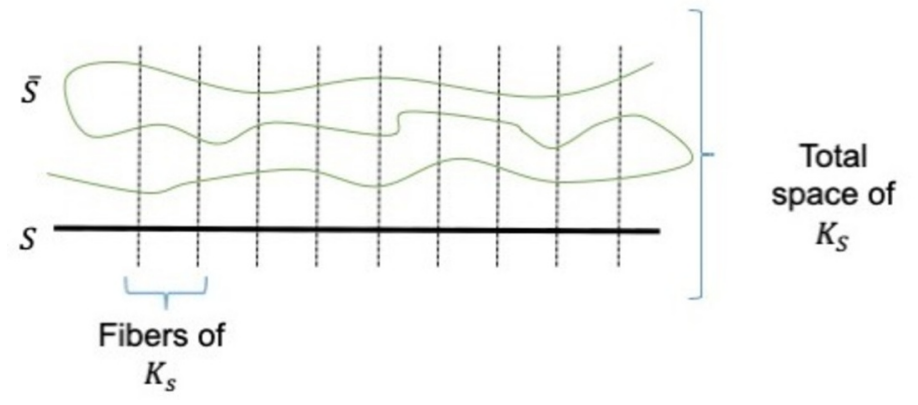

Figure 1. Top: spectral cover embedded in elliptically fibered Calabi-Yau (compact). Down: spectral cover embedded in the total space of canonical bundle (non-compact). The neighborhood of the zero section in $X$ is mapped to the total space of the canonical bundle [77].

\begin{tabular}{|c|c|c|}
\hline Spectral Cover & Zero Modes & Possible Yukawa Couplings \\
\hline$S_{4}=a_{4} X^{2}+a_{3} Y Z+a_{2} X Z^{2}+a_{0} Z^{4}$ & $\mathbf{1 6} a_{4}=0$ & $\mathbf{1 6 - 1 6 - 1 9}$ on $a_{3}=a_{4}=0$ \\
\hline & $\mathbf{1 0} a_{3}=0$ & $\mathbf{1 6} \mathrm{Bulk}$ \\
$S_{4}=Z\left(a_{3} Y+a_{2} X Z+a_{0} Z^{3}\right)$ & $\mathbf{1 6} a_{3}=0$ & $\mathbf{1 6 - 1 6 - 1 0}$ on $a_{3}=0$ \\
& $\mathbf{1 0} a_{3}=0$ & \\
\hline \multirow{3}{*}{$S_{4}=\left(a_{2} X+a_{0} Z^{2}\right)\left(b_{2} X+b_{0} Z^{2}\right)$} & $\mathbf{1 6} a_{2}=0$ & \multirow{2}{*}{$\mathbf{1 6} b_{2}=0$} \\
& $\mathbf{1 0} a_{2} b_{0}-a_{0} b_{2}=0$ & \\
\hline & $\mathbf{1 6} \mathrm{Bulk}-\mathbf{1 6}-\mathbf{1 0}$ on $a_{2}=b_{2}=0$ \\
& $\mathbf{1 6} \mathrm{Bulk}$ & $\mathbf{1 6 - 1 6 - 1 0}$ Bulk \\
\hline$S_{4}=Z^{2}\left(a_{2} X+a_{0} Z^{2}\right)$ & $\mathbf{1 0} \mathrm{Bulk}$ & \\
\hline$S_{4}=\left(a_{2} X+a_{0} Z^{2}\right)^{2}$ & $\mathbf{1 6} a_{2}=0$ & \multirow{2}{*}{$\mathbf{1 6 - 1 6 - 1 0}$ on $a_{2}=0$} \\
\hline
\end{tabular}

Table 2. SO(10) models possible Yukawa couplings. The middle column corresponds to the zero modes that can have non-zero Yukawa couplings. Note that for the last case there are three different possibilities. 


\begin{tabular}{|c|c|c|}
\hline Spectral Cover & Zero Modes & Possible Yukawa Couplings \\
\hline $\begin{array}{c}S_{5}= \\
a_{5} X Y+a_{4} X^{2} Z+a_{3} Y Z^{2}+a_{2} X Z^{3}+a_{0} Z^{5}\end{array}$ & $\begin{array}{c}\mathbf{1 0} a_{5}=0 \\
\mathbf{5} / \overline{\mathbf{5}} \\
a_{4} a_{3}^{2}-a_{2} a_{3} a_{5}+a_{0} a_{5}^{2}=0\end{array}$ & $\begin{array}{c}\mathbf{1 0 - 1 0 - 5} / \mathbf{1 0 -} \overline{\mathbf{5}}-\overline{\mathbf{5}} \text { on } \\
a_{5}=a_{4}=0 \\
a_{5}=a_{3}=0\end{array}$ \\
\hline$S_{5}=Z\left(a_{4} X^{2}+a_{3} Y Z+a_{2} X Z^{2}+a_{0} Z^{4}\right)$ & $\begin{array}{c}10 \text { Bulk } \\
10 a_{4}=0 \\
\mathbf{5} / \overline{\mathbf{5}} a_{4}=0 \\
\mathbf{5} / \overline{\mathbf{5}} a_{3}=0\end{array}$ & $\begin{array}{c}\mathbf{1 0 - 1 0 - 5} \text { on } a_{4}=0 \\
\mathbf{1 0 - \overline { 5 } - \overline { 5 }} \text { on } a_{4}=a_{3}=0\end{array}$ \\
\hline$S_{5}=Z\left(a_{2} X+a_{0} Z^{2}\right)\left(b_{2} X+b_{0} Z^{2}\right)$ & $\begin{array}{c}10 \text { Bulk } \\
10 a_{2}=0 \\
\mathbf{1 0} b_{2}=0 \\
\mathbf{5 / 5} a_{2}=0 \\
\mathbf{5 / 5} b_{2}=0 \\
\mathbf{5 / \overline { 5 }} a_{2} b_{0}-a_{0} b_{2}=0\end{array}$ & $\begin{array}{c}\mathbf{1 0 - 1 0 - 5} \text { on } \\
a_{2}=0 \text { and } b_{2}=0 \\
\mathbf{1 0 - 5 - 5} \text { on } \\
a_{2}=b_{2}=0\end{array}$ \\
\hline$S_{5}=\left(a_{2} X+a_{0} Z^{2}\right)\left(b_{3} Y+b_{2} X Z+b_{0} Z^{3}\right)$ & $\begin{array}{c}\mathbf{1 0} a_{2}=0 \\
\mathbf{1 0} b_{3}=0 \\
\mathbf{5} / \mathbf{5} b_{3}=0 \\
\mathbf{5} / \mathbf{5} c_{\Lambda^{2} V_{5}} 3.3 .2\end{array}$ & $\begin{array}{c}\text { 10-10-5 on } a_{2}=b_{3}=0 \\
\mathbf{1 0 - 5 - 5} \text { on } \\
c_{\Lambda^{2} V_{5}} \cap\left\{b_{3}=0\right\}\end{array}$ \\
\hline$S_{5}=Z\left(a_{2} X+a_{0} Z^{2}\right)^{2}$ & $\begin{array}{c}\mathbf{1 0} \text { Bulk } \\
\mathbf{1 0} a_{2}=0 \\
\mathbf{5} / \overline{\mathbf{5}} \text { Bulk } \\
\mathbf{5} / \overline{\mathbf{5}} a_{2}=0\end{array}$ & $\begin{array}{c}\mathbf{1 0 - 1 0 - 5} \text { on } a_{2}=0 \\
2 \times \mathbf{1 0 - 5}-\overline{5} \text { Bulk }\end{array}$ \\
\hline$S_{5}=Z^{2}\left(a_{3} Y+a_{2} X Z+a_{0} Z^{3}\right)$ & $\begin{array}{c}\mathbf{1 0} \text { Bulk } \\
10 a_{3}=0 \\
\mathbf{5} / \overline{\mathbf{5}} \text { Bulk } \\
\mathbf{5} / \overline{\mathbf{5}} a_{3}=0\end{array}$ & $\begin{array}{c}\mathbf{1 0 - 1 0 - 5} \text { on } a_{3}=0 \\
\mathbf{1 0 - 5 - 5} \text { on } a_{3}=0\end{array}$ \\
\hline$S_{5}=Z^{3}\left(a_{2} X+a_{0} Z^{2}\right)$ & $\begin{array}{l}10 \text { Bulk } \\
5 / \overline{5} \text { Bulk }\end{array}$ & $\begin{array}{c}\text { 10-10-5 Bulk } \\
3 \times \mathbf{1 0 - 5 - 5} \text { Bulk }\end{array}$ \\
\hline
\end{tabular}

Table 3. SU(5) models possible Yukawa couplings. The middle column corresponds to the zero modes that can have non-zero Yukawa couplings.

It is shown that a Fourier-Mukai functor can be represented by an integral transform,

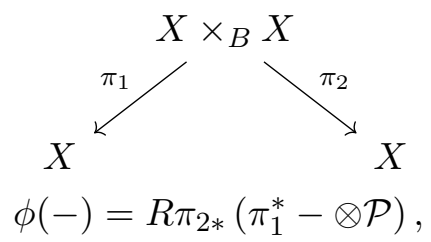

where $\mathcal{P}$ is the kernel of the integral transform, and it must satisfy certain conditions in order to $\Phi$ be an auto-equivalence. For the purposes of this paper, we restrict $\mathcal{P}$ to the Poincare sheaf,

$$
\mathcal{P}=\mathcal{I}_{\Delta} \otimes \pi_{1}^{*} \mathcal{O}(\sigma) \otimes \pi_{2}^{*} \mathcal{O}(\sigma) \otimes \rho^{*} K_{B}^{-1} .
$$


When $V$ is a stable degree zero bundle the Fourier-Mukai transform of $V$ is of the following form, ${ }^{4}$

$$
\Phi(V)=i_{S *} \mathcal{L}[-1]
$$

Where $S$ is a finite cover of the base i.e., spectral cover,

$$
\pi: S \stackrel{n: 1}{\longrightarrow} B
$$

and the sheaf $\mathcal{L}$ is called the spectral sheaf, which is a polarized rank one sheaf supported on $S$. Note that $S$ can be singular or even non-reduced. A more intuitive way to describe the Fourier-Mukai transform is as follows. When $V$ (which is stable, holomorphic and degree zero) restricted to a generic fiber (say $E$ ), it is equivalent to ${ }^{5}$

$$
\left.V\right|_{E} \simeq \mathcal{O}_{E}\left(\sigma-p_{1}\right) \oplus \cdots \oplus \mathcal{O}_{E}\left(\sigma-p_{n}\right),
$$

where $\sigma$ is the zero section, and $p_{n}$ are points on $E$ [96]. Spectral cover is simply the surface which is made by the points $p_{n}$ as one mover over the base.

Our purpose in this paper is to determine the Yukawa couplings in the effective theory from the spectral data. Remember if the map,

$$
H^{1}(V) \otimes H^{1}(V) \longrightarrow H^{2}\left(\Lambda^{2} V\right)
$$

is non-zero then the following Yukawa coupling is non-zero,

$$
H^{1}(V) \otimes H^{1}(V) \otimes H^{1}\left(\Lambda^{2} V^{*}\right) \longrightarrow \mathbb{C} .
$$

So our main task is finding the cohomologies $H^{*}(V)$ and $H^{*}\left(\Lambda^{2} V\right)$ in terms of the spectral data. For smooth spectral cover $S$, this is already have been done [76, 77]. The first novelty in this paper is finding the cohomologies for the singular spectral covers. We will see in the future that for singular $S$, there are possibilities for non-vanishing Yukawa couplings. This depends on the behavior of the spectral sheaves.

To find $H^{*}(V)$ we can use the Leray spectral sequence,

$$
E_{2}^{p, q}=H^{p}\left(R^{q} \pi_{*} V\right) \Rightarrow H^{p+q}(V) .
$$

So we should find $R^{q} \pi_{*} V$. This can be done using the following commutative diagram,

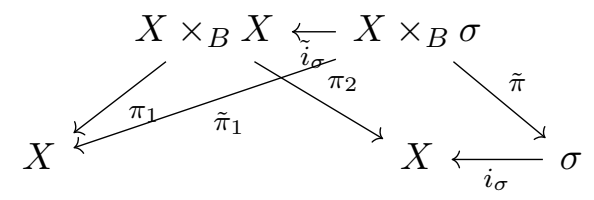

$$
\begin{aligned}
& R \pi_{*} V=R \tilde{\pi}_{*}\left(\tilde{\pi}_{1}^{*} V \otimes \mathcal{O}_{\sigma}\right) \\
& =R \tilde{\pi}_{*}\left(\tilde{\pi}_{1}^{*} V \otimes L \tilde{i}_{\sigma}^{*} \mathcal{P}\right) \\
& =R \tilde{\pi}_{*} \circ L \tilde{i}_{\sigma}^{*}\left(\pi_{1}^{*} V \otimes \mathcal{P}\right) \\
& =L i_{\sigma}^{*} R \pi_{2 *}\left(\pi_{1}^{*} V \otimes \mathcal{P}\right) \\
& =L i_{\sigma}^{*} \Phi(V)=L i_{\sigma}^{*}\left(i_{S *} \mathcal{L}\right)[-1],
\end{aligned}
$$

\footnotetext{
${ }^{4}$ Even when the restriction of $V$ on generic elliptic fiber is semistable and degree zero, the Fourier-Mukai transform of $V$ has this general form. But for physical models this is not enough.

${ }^{5}$ To be precise S-equivalent [95].
} 
where we have used the flattness of the map $\tilde{\pi}$ which is the consequence of the flattness of $\pi$. This is of course not a new result [65].

To compute $H^{*}\left(\Lambda^{2} V\right)$, we need to know, as before, the derived pushforward $R \pi_{*} \Lambda^{2} V$. For this, we use a nice property of Fourier-Mukai transforms (defined via Poincare sheaf as the kernel) which exchanges the tensor product with the Pontrjagin product. More concretely, consider a single smooth elliptic curve $E$. Then there is a morphism $m$ which corresponds to the abelian group action on the elliptic curve [82],

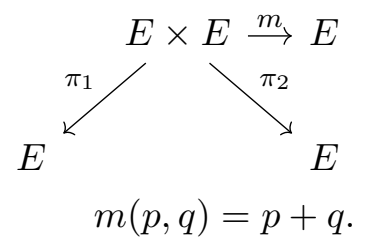

Then the Pontrjagin product is defined as the derived pushforward of $m$,

$$
V * W:=R m_{*}\left(\pi_{1}^{*} V \otimes \pi_{2}^{*} W\right)
$$

where $V$ and $W$ are sheaves over the elliptic curve $E$. Over $E$, Fourier-Mukai functor satisfy the following identity

$$
\Phi(V) * \Phi(W)=\Phi(V \otimes W)[-1]
$$

One needs a similar identity over the elliptically fibered $X$. However, the morphism $m$ is not well defined for the total fibration. Because there are singular fibers, and the group action is not well defined when the points $p$ and $q$ correspond to the singular point of the elliptic fiber. Therefore if the spectral covers of $V$ and $W$ hit the singular point, this identity will not work.

The good news is that we need the spectral cover of the antisymmetric product. So the addition of the singular point with itself doesn't contribute. In addition, for the cohomology of $\Lambda^{2} V$, only the restriction of the $\Phi\left(\Lambda^{2} V\right)$ on the zero section $\sigma$ contributes. So even if the spectral cover of $V$ hits the singular points of the fibers, they do not contribute in the $H^{*}\left(\Lambda^{2} V\right),{ }^{6}$

$$
\begin{aligned}
L i_{\sigma}^{*} \Phi\left(\Lambda^{2} V\right) & =L i_{\sigma}^{*}\left(\Phi(V) *_{A} \Phi(V) \otimes K_{B}^{-1}[+1]\right) \\
& =L i_{\sigma}^{*}\left(\left(i_{S *} \mathcal{L}\right) *_{A}\left(i_{S *} \mathcal{L}\right) \otimes K_{B}^{-1}[-1]\right),
\end{aligned}
$$

where by $*_{A}$ we mean "antisymmetrized" version of the Pontrjagin product which hopefully will be more clear in the examples.

\footnotetext{
${ }^{6}$ There is one caveat in this argument when the spectral cover has non-reduced vertical fiber. But again, we are only care about the restriction of the $\Phi\left(\Lambda^{2} V\right)$ to the zero section. So it still fine to use this formula.
} 
To prove this we use the following commutative diagrams, ${ }^{7}$

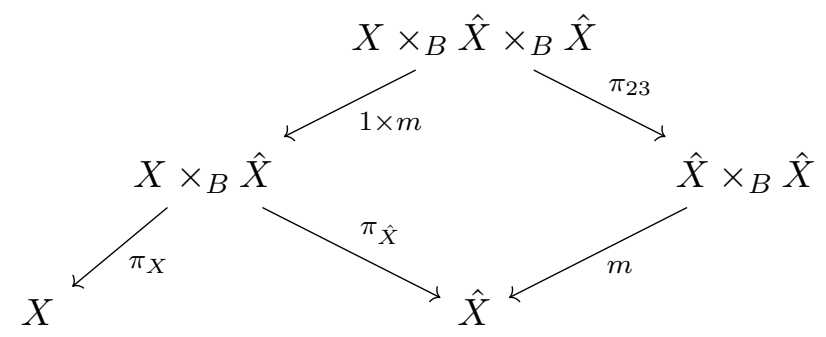

From here we can see,

$$
R m_{*} \circ R \pi_{23 *} \simeq R \pi_{\hat{X} *} \circ R(1 \times m)_{*} .
$$

The following diagram is also useful,

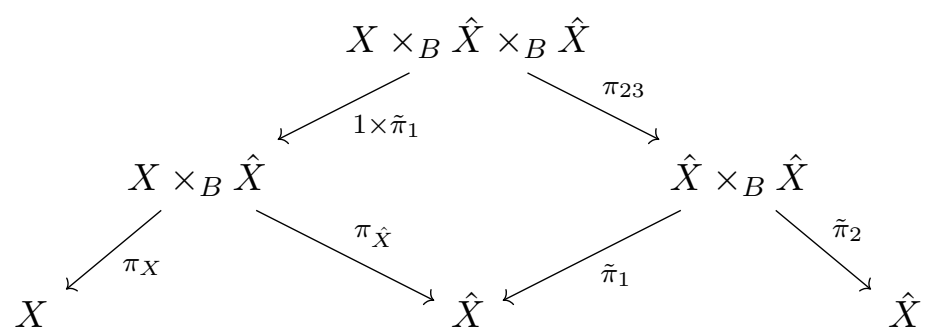

Form here one can see,

$$
\tilde{\pi}_{1}^{*} \circ R \pi_{\hat{X} *} \simeq R \pi_{23 *} \circ\left(1 \times \tilde{\pi}_{1}\right)^{*}
$$

Therefore,

$$
\tilde{\pi}_{1}^{*} \Phi(V)=R \pi_{23 *} \circ\left(1 \times \tilde{\pi}_{1}\right)^{*}\left(\pi_{X}^{*} V \otimes \mathcal{P}\right) .
$$

So now let us compute $\Phi(V) * \Phi(W)$,

$$
\begin{aligned}
\tilde{\pi}_{1}^{*} \Phi(V) \otimes \tilde{\pi}_{2}^{*} \Phi(W) & =R \pi_{23 *} \circ\left(1 \times \tilde{\pi}_{1}\right)^{*}\left(\pi_{X}^{*} V \otimes \mathcal{P}\right) \otimes \tilde{\pi}_{2}^{*} \Phi(W) \\
& =R \pi_{23 *}\left(\left(1 \times \tilde{\pi}_{1}\right)^{*}\left(\pi_{X}^{*} V \otimes \mathcal{P}\right) \otimes \pi_{23}^{*} \tilde{\pi}_{2}^{*} \Phi(W)\right),
\end{aligned}
$$

hence,

$$
\begin{aligned}
R m_{*}\left(\tilde{\pi}_{1}^{*} \Phi(V) \otimes \tilde{\pi}_{2}^{*} \Phi(W)\right) & =R m_{*} \circ R \pi_{23 *}\left(\left(1 \times \tilde{\pi}_{1}\right)^{*}\left(\pi_{X}^{*} V \otimes \mathcal{P}\right) \otimes \pi_{23}^{*} \tilde{\pi}_{2}^{*} \Phi(W)\right) \\
& =R \pi_{\hat{X} *} \circ R(1 \times m)_{*}\left(\left(1 \times \tilde{\pi}_{1}\right)^{*}\left(\pi_{X}^{*} V \otimes \mathcal{P}\right) \otimes \pi_{23}^{*} \tilde{\pi}_{2}^{*} \Phi(W)\right) \\
& =R \pi_{\hat{X} *}\left(\pi_{X}^{*} V \otimes R(1 \times m)_{*}\left(\pi_{12}^{*} \mathcal{P} \otimes \pi_{23}^{*} \tilde{\pi}_{2}^{*} \Phi(W)\right)\right)
\end{aligned}
$$

Now there are two other relations that should be used,

$$
\begin{aligned}
\pi_{23}^{*} \circ \tilde{\pi}_{2}^{*} & \simeq \pi_{13}^{*} \circ \pi_{\hat{X}}^{*}, \\
\pi_{12}^{*} \mathcal{P} & =(1 \times m)^{*} \mathcal{P} \otimes \pi_{13}^{*} \mathcal{P},
\end{aligned}
$$

\footnotetext{
${ }^{7}$ Here $\hat{X}$ is the Jacobian fibration, which is isomorphic to $X$.
} 
where the first relation is coming from the simple relations between the projection morphisms, and the second one is true for the Poincare sheaf on any abelian variety. With the help of these two we simplify the last result above,

$$
R m_{*}\left(\tilde{\pi}_{1}^{*} \Phi(V) \otimes \tilde{\pi}_{2}^{*} \Phi(W)\right)=R \pi_{\hat{X} *}\left(\pi_{X}^{*} V \otimes \mathcal{P} \otimes R(1 \times m)_{*} \pi_{13}^{*}\left(\mathcal{P}^{*} \otimes \pi_{\hat{X}}^{*} \Phi(W)\right)\right) .
$$

The following commutative diagram,

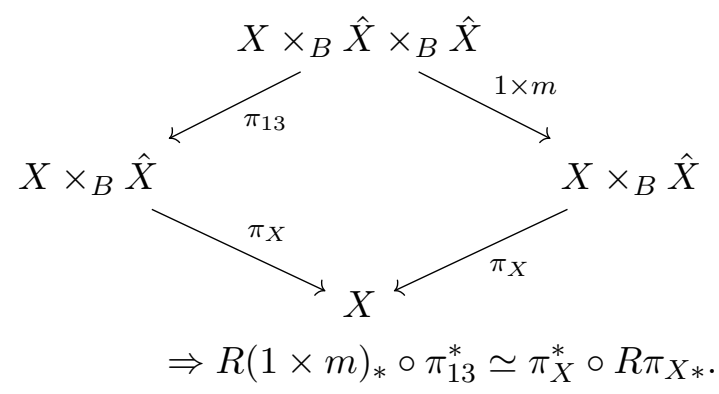

Therefore,

$$
\begin{aligned}
R m_{*}\left(\tilde{\pi}_{1}^{*} \Phi(V) \otimes \tilde{\pi}_{2}^{*} \Phi(W)\right) & =R \pi_{\hat{X} *}\left(\pi_{X}^{*} V \otimes \mathcal{P} \otimes p i_{X}^{*} \circ R \pi_{X *}\left(\mathcal{P}^{*} \otimes \pi_{\hat{X}}^{*} \Phi(W)\right)\right) \\
& =R \pi_{\hat{X}_{*}}\left(\pi_{X}^{*} V \otimes \mathcal{P} \otimes \pi_{X}^{*} W \otimes K_{B}[-1]\right),
\end{aligned}
$$

where in the last line we used the inverse Fourier-Mukai functor i.e., $R \pi_{X *}\left(\mathcal{P}^{*} \otimes \pi_{\hat{X}}^{*} \Phi(W)\right)=$ $W \otimes K_{B}[-1]$. Then the final result is,

$$
\Longrightarrow R m_{*}\left(\tilde{\pi}_{1}^{*} \Phi(V) \otimes \tilde{\pi}_{2}^{*} \Phi(W)\right)=\Phi(V \otimes W) \otimes K_{B}[-1] .
$$

Of course the formula (2.35) is wrong! But If we choose $W=V$ and antisymmetrize the tensor product then it can be used for computing $R \pi_{*} \Lambda^{2} V$.

\subsection{Degree two spectral cover}

As a warm-up let us study the spectral cover corresponding to $\mathrm{SU}(2)$ bundles. Of course, this theory doesn't have a Yukawa coupling, but it is useful for the later parts to work out its Pontrjagin product.

Consider the smooth degree two spectral cover,

$$
\begin{aligned}
\Phi(V) & =i_{S_{2} *} \mathcal{L}_{2}[-1], \\
S_{2} & =a_{2} X+a_{0} Z^{2},
\end{aligned}
$$

where $a_{2}$ and $a_{0}$ are generic polynomials in the base $B_{2}$. Generally, we are interested in the cohomologies $H^{*}(V)$ and $H^{*}\left(\Lambda^{2} V\right)$. The former can be easily computed as,

$$
H^{i}(V)=H^{i-1}\left(R^{1} \pi_{*} V\right)=H^{i-1}\left(\left.\mathcal{L}_{2}\right|_{\sigma \cap S_{2}}\right) .
$$

For $H^{*}\left(\Lambda^{2} V\right)$, since $\Lambda^{2} V=\mathcal{O}_{X}$ (for any $\mathrm{SU}(2)$ bundle) $H^{i}\left(\Lambda^{2} V\right)=H^{i}\left(\mathcal{O}_{X}\right)$. So in principle, one doesn't need to use spectral data, however as mentioned before, it is instructive to compute the Pontrjagin product. Since the antisymmetric Pontrjagin 
product is needed, one should "multiply" the line bundles on the opposite sheets of the double cover to get a sheaf over the zero section. In other words, suppose the double cover intersects a generic fiber at two points $P_{1}$ and $P_{2}$, since the bundle is $\mathrm{SU}(2)$ these points should "add-up" to zero,

$$
P_{1} \boxplus P_{2}=0 .
$$

The antisymmetric Pontrjagin product basically take the sheaves supported over the points $P_{1}$ and $P_{2}$ and generates a sheaf over the "zero section" 0. Globally one can find this sheaf over the zero section as $\operatorname{Det}\left(\pi_{*} i_{S_{2} *} \mathcal{L}_{2}\right)$. This is because the pushforward to the base is a direct sum of two line bundles corresponding to the two sheets of the double cover [95]. Therefore the determinant gives the antisymmetrized product. ${ }^{8}$ So let us check this

$$
i_{S_{2} *} \mathcal{L}_{2} \star_{A} i_{S_{2} *} \mathcal{L}_{2}=i_{\sigma *} \operatorname{Det}\left(\pi_{*} i_{S_{2} *} \mathcal{L}_{2}\right) \Longrightarrow \Phi\left(\Lambda^{2} V\right)=i_{\sigma *} \operatorname{Det}\left(\pi_{*} i_{S_{2} *} \mathcal{L}_{2}\right) \otimes K_{B}^{-1}[-1]
$$

By the usual arguments one can show,

$$
\begin{aligned}
R \pi_{*} \Lambda^{2} V & =\operatorname{Li}_{\sigma}^{*} i_{\sigma *} \operatorname{Det}\left(\pi_{*} i_{S_{2} *} \mathcal{L}_{2}\right) \otimes K_{B}^{-1}[-1] \\
& =\operatorname{Det}\left(\pi_{*} i_{S_{2} *} \mathcal{L}_{2}\right) \otimes K_{B}^{-2} \oplus \operatorname{Det}\left(\pi_{*} i_{S_{2} *} \mathcal{L}_{2}\right) \otimes K_{B}^{-1}[-1]
\end{aligned}
$$

On the other hand, it can be shown for a degree zero $\mathrm{SU}(2)$ bundle one has the following relation $\mathrm{A}$,

$$
c_{1}\left(\pi_{*} i_{S_{2} *} \mathcal{L}_{2}\right)=2 c_{1}\left(K_{B}\right) \Longrightarrow \operatorname{Det}\left(\pi_{*} i_{S_{2} *} \mathcal{L}_{2}\right)=K_{B}^{2}
$$

Hence,

$$
\begin{aligned}
& R^{0} \pi_{*} \Lambda^{2} V=\mathcal{O}_{B}, \\
& R^{1} \pi_{*} \Lambda^{2} V=K_{B} .
\end{aligned}
$$

This is exactly the derived pushforward formula for the trivial bundle $\mathcal{O}_{X}$. Consistent with the expectation $\Lambda^{2} V=\mathcal{O}_{X}$.

\subsection{Caveats}

Before digging into the main body of calculations, we should stop to explain a few caveats. First, suppose $\left(\mathcal{S}_{V}, \mathcal{L}_{V}\right)$ are the spectral data of a degree zero coherent $V$, with $S_{V}$ being a smooth spectral cover. As proved in [59,60] ( also look at Proposition 6.64 in [82] ), that $V$ is stable relative to the polarization $J=a \sigma+b H_{B}{ }^{9}$ (for large enough $b \underline{0}$ ) if and only if the spectral sheaf $\mathcal{L}_{V}$ is stable as a coherent sheaf on $\mathcal{S}_{V}$ relative to the polarization $J^{\prime}=\left.\pi^{*} H_{B}\right|_{\mathcal{S}_{V}}$. When $V$ is locally free (vector bundle), then $\mathcal{L}_{V}$ must be a line bundle (this is well known and can be found in [82]). Therefore a vector bundle $V$ with smooth spectral cover is always stable for large enough $b$. However, in this paper, we do not consider smooth spectral covers necessarily, and one may concern whether bundles are stable or not. There

\footnotetext{
${ }^{8}$ More precisely, the pushforward $\pi_{*} \mathcal{O}_{S_{2}}=\mathcal{O}_{B} \oplus \mathcal{O}_{B}\left(-\frac{R}{2}\right)$ where $R$ is the branch locus. The first summand corresponds to the part of $\mathcal{O}_{S_{2}}$ which is even under the involution (exchange of the sheets) of the double cover $S_{2}$, and the second summand is the odd part.

${ }^{9} a$ and $b$ are positive integers and $H_{B}$ is an ample divisor in the base $B$.
} 
are no general criteria for the stability of vector bundles with singular spectral covers. In other words, one should check case by case whether the bundle she or he is studying is indeed stable. This doesn't ruin the generality of the results proven in this paper. Since as much as the author is aware, it is possible to have stable vector bundles with all spectral cover geometries considered here. The most well-known example is the tangent bundle with a completely degenerate spectral cover, and hence highly singular, but yet it is quite a well-defined smooth, stable bundle and corresponds to a supersymmetric vacuum.

The second issue is the exact meaning of the singularity of the spectral data. Both $\mathcal{S}_{V}$ and $\mathcal{L}_{V}$ can be singular. When $\mathcal{S}_{V}$ is singular, it is always possible (at least for the cases considered here) to smooth the spectral cover. In other words, there are smooth representatives in the divisor class $\left[\mathcal{S}_{V}\right]$. The divisor class is fixed by the bundle topology (Chern classes of $V$ ), and for the same topology of bundle, spectral cover can have different geometries. However, the smoothing process can be obstructed physically. This depends on whether, after smoothing, the spectral sheaf "deforms" into a holomorphic line bundle or not (and hence the corresponding bundle becomes holomorphic). This is a non-trivial condition and, as is shown in [80], not always satisfied. The situation is equivalent to in intersecting brane models [90, 91]. In these models, the smoothing (recombination) of the intersection branes may be obstructed by the sheaves living on the branes. This holomorphicity constraint can be used to stabilize the bundle, or complex structure moduli [92, 93]. However, this is not the main concern for us here because we do not care about the moduli stabilization in this work. On the other hand, the spectral sheaf can be singular too. However, we demand that $V$ always be smooth (vector bundle), even though one can still make sense of a heterotic model with torsion-free sheaves instead of vector bundles. The smoothness of $V$ puts strong constraints on the singularities of the spectral sheaf. When $\mathcal{S}_{V}$ is smooth, the spectral sheaf must be a line bundle, as mentioned before. But when $\mathcal{S}_{V}$ is singular (so reducible), the spectral sheaf on each component can be singular and, the singularities are allowed only to be on the intersection loci of the components of the spectral cover (this is the result of the smoothness constraint of $V$ ). These singularities do not contribute to the Yukawa couplings of the effective theory, as can be checked in the examples. So we assumed in this paper that the restriction of the spectral sheaf over each component is smooth. The argument is not hard.

\section{Spectral covers without vertical components}

In this section, the technology explained in the previous section is used to study more realistic models. For now, let us restrict ourselves to spectral covers, either singular or not, that have no vertical components. Those cases are studied in the next section.

\section{$3.1 \quad E_{6}$ GUT models}

For $E_{6}$ GUT models, one needs to put a $\mathrm{SU}(3)$ bundle (denoted by $V$ as usual) in the internal space. Therefore the spectral cover in this case is a degree cover of the base surface. There are only two possibility for the degree three spectral cover without vertical component, as shown in figure 2. 

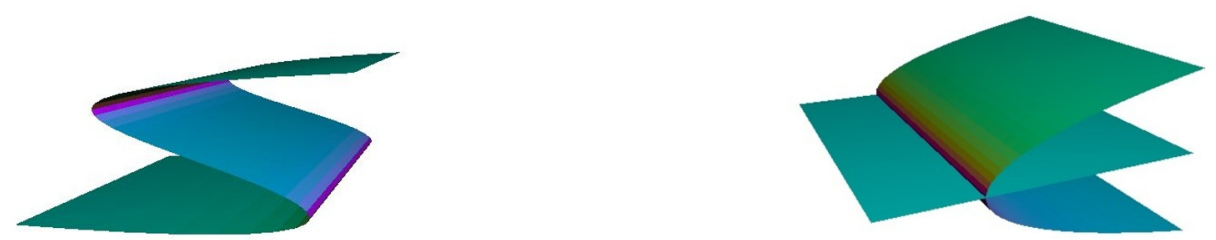

Figure 2. Left: smooth degree three spectral cover. Right: reducible degree three spectral cover, with one component isomorphic to the base manifold.

\subsubsection{Smooth spectral cover}

In this subsection we compute the cohomology of $V$ and $\Lambda^{2} V$ when spectral cover is given by,

$$
S_{3}=a_{3} Y+a_{2} X Z+a_{0} Z^{3} .
$$

As usual, for $H^{*}(V)$ we need $R \pi_{*} V$, which from our experience of smooth degree two spectral cover is,

$$
R \pi_{*} V=L i_{\sigma}^{*} \mathcal{L}_{3}[-1]
$$

Therefore $H^{p}(V)=H^{p-1}\left(R^{1} \pi_{*} V\right)=H^{p-1}\left(\left.\mathcal{L}_{3}\right|_{S_{3} \cap \sigma}\right)$. Usually the curve $S_{3} \cap \sigma$, which is given by the equation $a_{3}=0$ in the base of $X$, is called the matter curve for obvious reasons.

What can be said about $R \pi_{*} \Lambda^{2} V$ ? As for $V, \Lambda^{2} V$ also satisfy the following identity,

$$
R \pi_{*} \Lambda^{2} V=\left.\Phi\left(\Lambda^{2} V\right)\right|_{\sigma}
$$

Now the formula of the previous section can be used,

$$
\left.\Phi\left(\Lambda^{2} V\right)\right|_{\sigma}=\left.R m_{*}\left(\pi_{1}^{*} \Phi(V) \otimes_{A} \pi_{2}^{*} \Phi(V)\right)\right|_{\sigma} \otimes K_{B}^{-1}[+1],
$$

where the subscript $A$ in the right hand side means antisymmetrized product. In addition, we are looking for restriction on the zero section. To see how this can happen, consider a generic elliptic fiber. Let $P_{1}, P_{2}$ and $P_{3}$ be the intersection of the fiber with $S_{3}$. Then by definition,

$$
P_{1} \boxplus P_{2} \boxplus P_{3}=0 .
$$

The points that correspond to the intersection of the same fiber with the spectral cover of $\Lambda^{2} V$ will be,

$$
P_{1} \boxplus P_{2}, \quad P_{1} \boxplus P_{2}, \quad P_{2} \boxplus P_{3} .
$$

So the restriction on the zero section $\sigma$ is non-zero if one of these points, say $P_{1} \boxplus P_{2}$, is the zero point of the elliptic curve so,

$$
P_{1} \boxplus P_{2}=0, \quad P_{3}=0 .
$$



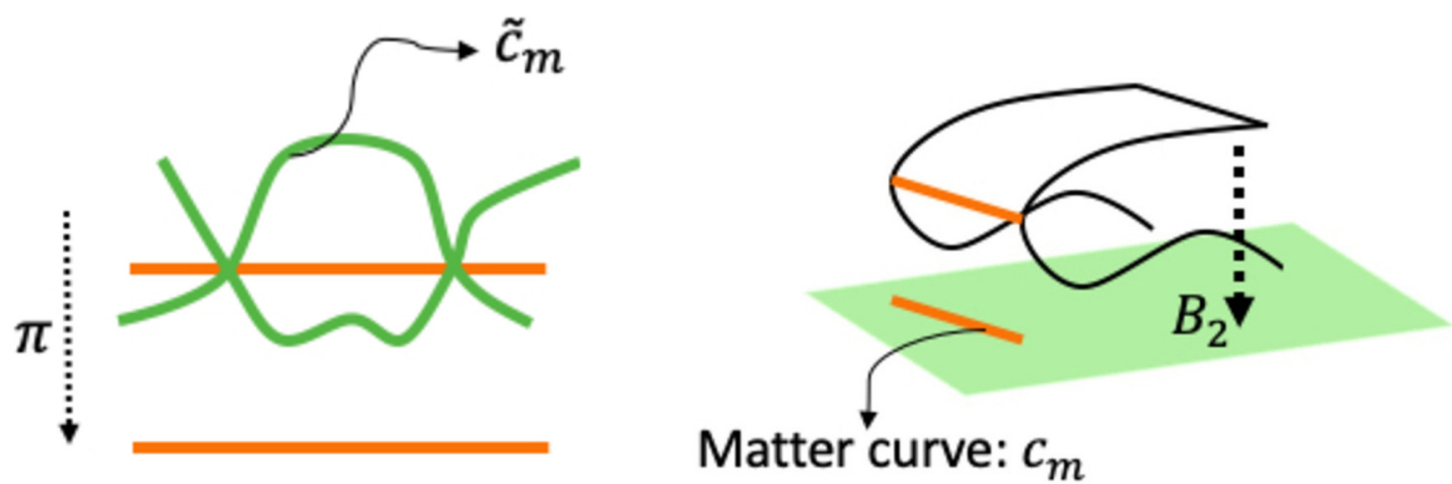

Figure 3. Left: the preimage of the matter curve inside the spectral cover. This is a reducible cover of $c_{m}$, where one component is isomorphic to the matter curve (the orange line) and one component is a double cover of the matter curve (green line). Right: the matter curve, i.e., the intersection locus of the zero section and the spectral cover.

So $\left.\Phi\left(\Lambda^{2} V\right)\right|_{\sigma}$ is only non-zero over the matter curve $a_{3}=0 .{ }^{10}$ So the bottom line is,

$$
R \pi_{*} \Lambda^{2} V=\left.\Phi\left(\Lambda^{2} V\right)\right|_{\sigma}=R m_{*}\left(\left.\left.\pi_{1}^{*} \mathcal{L}_{3}\right|_{\tilde{c}} \otimes_{A} \pi_{2}^{*} \mathcal{L}_{3}\right|_{\tilde{c}}\right) \otimes K_{B}^{-1}[-1],
$$

where $\tilde{c}$ is the double cover of the matter curve $a_{3}=0$, and defined in the following way,

$$
\left.S_{3}\right|_{a_{3}=0}=\left.Z\left(a_{2} X+a_{0} Z^{2}\right)\right|_{a_{3}=0}=c \cup \tilde{c} .
$$

So to evaluate the right hand side of (3.9) one needs to multiply the line bundle $\mathcal{L}$ on different sheets of $\tilde{c}$. The final result is the following,

$$
R \pi_{*} \Lambda^{2} V=R m_{*}\left(\left.\pi_{1}^{*} \mathcal{L}_{3}\right|_{\tilde{c}} \otimes_{A} \pi_{2}^{*} \mathcal{L}_{3} \mid \tilde{c}\right) \otimes K_{B}^{-1}[-1]=\operatorname{Det}\left[\pi_{*} \mathcal{L}_{3} \mid \tilde{c}\right] \otimes K_{B}^{-1}[-1] .
$$

To see this, note that the pushforward $\pi_{*} \mathcal{O}_{\tilde{c}}=\mathcal{O}_{c} \oplus \mathcal{O}\left(-\frac{R}{2}\right)$ locally. Where $R$ is the branch divisor of the $\tilde{c}$. Then each factor $\mathcal{O}_{c}$ and $\mathcal{O}\left(-\frac{R}{2}\right)$ corresponds to line bundles over each sheet. Therefore taking the determinant of $\pi_{*} \mathcal{O}_{\tilde{c}}$ is the same as multiplying the line bundles over each sheet.

So now it is clear that the Yukawa couplings 27-27-27 are zero. The reason is $R^{1} \pi_{*} V$ receives contribution from $\left.\mathcal{L}\right|_{c}$, but $R^{1} \pi_{*} \Lambda^{2} V$ depends on $\left.\mathcal{L}\right|_{\tilde{c}}$. So the following map must be vanishing,

$$
H^{0}\left(R^{1} \pi_{*} V\right) \otimes H^{0}\left(R^{1} \pi_{*} V\right) \longrightarrow_{0} H^{0}\left(R^{1} \pi_{*} \Lambda^{2} V\right) .
$$

In addition, the image of the product of the cohomologies on the left lives inside $H^{0}\left(\left.\mathcal{L}_{3}\right|_{c_{m}}{ }^{\otimes 2}\right)$, and the cohomology on the right hand side is given by $H^{0}\left(\left.\pi_{*} \mathcal{L}_{3}\right|_{\tilde{c}_{m}}\right)$. However, it is shown in the A that $c_{1}\left(\pi_{*} V\right)=3 K_{B}$ then

$$
c_{1}\left(i_{c_{m}}^{*} \pi_{*} V\right)=c_{1}\left(\left.\mathcal{L}_{3}\right|_{c_{m}}\right) c_{1}\left(\left.\pi_{*} \mathcal{L}_{3}\right|_{\tilde{c}_{m}}\right)=\left.3 K_{B}\right|_{c_{m}},
$$

\footnotetext{
${ }^{10}$ This is of course not surprising, because for $\mathrm{SU}(3)$ bundles $\Lambda^{2} V=V^{*}$. However we prefer the approach mentioned above because it can be generalized to higher rank bundles. It is not very hard to show that
}

$$
S_{3}\left(\Lambda^{2} V\right)=-a_{3} Y+a_{2} X Z+a_{0} Z^{3} .
$$


where $i_{c_{m}}: c_{m} \hookrightarrow X$ is just the inclusion of the (preimage of) the matter curve inside the Calabi-Yau X. Using this relation it is clear that the map (3.12) is cannot be non-zero generically.

This has a clear F-theory interpretation. There are three ways that one get Yukawa couplings in local F-theory models [49]: 1) triple intersection of 7-branes, 2) interaction of the bulk zero modes and the matter zero modes (which are localized over matter curves), 3) Interaction between bulk zero modes with each other. In the current model, all of the matter content of the effective theory are coming from the zero modes that are living over the curve $c_{m}$, and hence it is impossible to get non-zero Yukawa couplings from them. More precisely the F-theory geometry (after stable degeneration) is

$$
\begin{aligned}
Y^{2}+X^{3}+\alpha_{1} v X Y & +\left(\alpha_{2} v^{3}+a_{3} u v^{2}\right) Y+\left(\alpha_{4} v^{4}+a_{2} u v^{3}\right) X \\
& +\left(\alpha_{6} v^{6}+a_{0} u v^{5}\right)
\end{aligned}
$$

where $(X, Y, u, v)$ are homogeneous coordinates of $\mathbb{W P}^{2,3,1,1}$, and $v=0$ is the locus of the GUT brane [86]. The singularity over $v=0$ is generically $E_{6}$, but over the curve $a_{3}=0$ the singularity enhances to $E_{7}$, and directly from F-theory it can be seen there are $\mathbf{2 7}$ and $\overline{\mathbf{2 7}}$ hypermultiplets living in this curve. The singularity enhances to $E_{8}$ over the intersection locus $v=a_{3}=a_{2}=0$, however this doesn't correspond to a Yukawa couplings [78].

\subsubsection{Reducible reduced spectral cover}

The spectral cover in this case is a triple cover of the base, and the only possible reducible spectral cover (with one component parallel to the base) is (figure 2)

$$
S_{3}=Z\left(a_{2} X+a_{0} Z^{2}\right) .
$$

Without loss of generality the spectral sheaf can be written as [88],

$$
0 \longrightarrow i_{\sigma *} \mathcal{L}_{1} \longrightarrow i_{S *} \mathcal{L}_{3} \longrightarrow i_{S_{2}} \mathcal{L}_{2} \longrightarrow 0
$$

where $\mathcal{L}_{1}$ is a line bundle in base, $\mathcal{L}_{2}$ is a torsion free sheaf on $S_{2}$ with possible singularities over the intersection $\sigma \cap S_{2}=\left\{a_{2}=0\right\}$ (but as mentioned before we assume there are no singularities in these sheaves). The extension morphism of this short exact sequence is localized over $\left\{a_{2}=0\right\}$,

$$
\operatorname{Ext}^{1}\left(i_{S_{2} *} \mathcal{L}_{2}, i_{\sigma *} \mathcal{L}_{1}\right)=\operatorname{Hom}\left(\left.\mathcal{L}_{2}\right|_{\left\{a_{2}=0\right\}},\left.\mathcal{L}_{1}\right|_{\left\{a_{2}=0\right\}} \otimes \mathcal{O}\left(-S_{2}\right)\right) .
$$

Next, one needs to find $\pi_{1}^{*} i_{S *} \mathcal{L} \otimes_{A} \pi_{2}^{*} i_{S *} \mathcal{L}$. To do this we can antisymmetrize the sequence above

$$
\begin{aligned}
0 \longrightarrow \pi_{1}^{*} i_{\sigma *} \mathcal{L}_{1} \otimes_{S} \pi_{2}^{*} i_{\sigma *} \mathcal{L}_{1} \longrightarrow & \pi_{1}^{*} i_{\sigma *} \mathcal{L}_{1} \otimes \pi_{2}^{*} i_{S *} \mathcal{L} \\
& \stackrel{\downarrow}{\downarrow}{ }_{1}^{*} i_{S *} \mathcal{L} \otimes_{A} \pi_{2}^{*} i_{S *} \mathcal{L} \longrightarrow \pi_{1}^{*} i_{S_{2} *} \mathcal{L}_{2} \otimes_{A} \pi_{2}^{*} i_{S_{2} *} \mathcal{L}_{2} \longrightarrow 0
\end{aligned}
$$


or equivalently,

$$
0 \longrightarrow \pi_{1}^{*} i_{\sigma *} \mathcal{L}_{1} \otimes \pi_{2}^{*} i_{S_{2} *} \mathcal{L}_{2} \longrightarrow \pi_{1}^{*} i_{S *} \mathcal{L} \otimes_{A} \pi_{2}^{*} i_{S *} \mathcal{L} \longrightarrow \pi_{1}^{*} i_{S_{2} *} \mathcal{L}_{2} \otimes_{A} \pi_{2}^{*} i_{S_{2} *} \mathcal{L}_{2} \longrightarrow 0
$$

Applying the $R m_{*}$ functor on this sequence gives a long exact sequence, but fortunately this is simple in the current situation,

$$
0 \longrightarrow \mathcal{L}_{1} \otimes i_{S_{2} *} \mathcal{L}_{2} \longrightarrow R^{0} m_{*}\left(\pi_{1}^{*} i_{S *} \mathcal{L} \otimes_{A} \pi_{2}^{*} i_{S *} \mathcal{L}\right) \longrightarrow i_{\sigma *}\left(\operatorname{Det}_{*} \mathcal{L}_{2}\right) \longrightarrow 0
$$

Note that the spectral cover is unchanged,

$$
S_{\Lambda^{2} V}=S_{3}
$$

Now let us apply the left derived functor $L i_{\sigma}^{*}$ to the sequence above

$$
\begin{gathered}
0 \longrightarrow L^{-1} i_{\sigma}^{*} R^{0} m_{*}\left(\pi_{1}^{*} i_{S *} \mathcal{L} \otimes_{A} \pi_{2}^{*} i_{S *} \mathcal{L}\right) \longrightarrow\left(\operatorname{Det}_{*} \mathcal{L}_{2}\right) \otimes K_{B}^{-1} \\
\longrightarrow i_{\left\{a_{2}=0\right\} *}\left(\mathcal{L}_{1} \otimes \mathcal{L}_{2}\right) \longrightarrow L^{0} i_{\sigma}^{*} R^{0} m_{*}\left(\pi_{1}^{*} i_{S *} \mathcal{L} \otimes_{A} \pi_{2}^{*} i_{S *} \mathcal{L}\right) \longrightarrow \operatorname{Det}_{*} \mathcal{L}_{2} \longrightarrow
\end{gathered}
$$

Finding the exact result depends on the coboundary map which is induced by the extension of the (3.20) restricted on $\left\{a_{2}=0\right\}$. As will be clear in the examples, the coboundary map is not necessarily zero, and the zero modes $i_{\left\{a_{2}=0\right\}_{*}}\left(\mathcal{L}_{1} \otimes \mathcal{L}_{2} \otimes K_{B}^{-1}\right)$ do not necessarily inject into $R^{1} \pi_{*} \Lambda^{2} V$. To compute $H^{1}(V), R^{1} \pi_{*} V$ is also needed

$$
\begin{gathered}
R \pi_{*} V=\mathcal{L}_{1} \otimes K_{B}^{-1} \oplus \mathcal{F}[+1], \\
0 \longrightarrow \mathcal{L}_{1} \longrightarrow \mathcal{F} \longrightarrow i_{\left\{a_{2}=0\right\} *} \mathcal{L}_{2} \longrightarrow 0
\end{gathered}
$$

By Leray spectral sequence, it is possible to compute the cohomologies in terms of these pushforwad results derived so far. For $V$ one gets

$$
0 \longrightarrow H^{1}\left(\pi_{*} V\right) \rightarrow H^{1}(V) \rightarrow H^{0}\left(R^{1} \pi_{*} V\right) \stackrel{\alpha}{\longrightarrow} H^{2}\left(\pi_{*} V\right) \rightarrow \ldots
$$

and for $\Lambda^{2} V$,

$$
H^{0}\left(R^{1} \pi_{*} \Lambda^{2} V\right) \longrightarrow H^{2}\left(\pi_{*} \Lambda^{2} V\right) \longrightarrow H^{2}\left(\Lambda^{2} V\right) \rightarrow H^{1}\left(R^{1} \pi_{*} \Lambda^{2} V\right) \rightarrow 0
$$

So $H^{1}\left(\pi_{*} V\right)$ gets injected into $H^{1}(V)$. In addition, the homomorphism $\alpha$ lives in

$$
\alpha \in \operatorname{Ext}^{2}\left(\mathcal{F}, \mathcal{L}_{1} \otimes K_{B}^{-1}\right) .
$$

$\alpha$ should be zero, because at the end of the day, it should be induced by the extension morphism of the original defining sequence, and by checking the extension group above, $\alpha$ cannot be induced by the original sequence. Therefore,

$$
H^{1}(V)=H^{1}\left(\pi_{*} V\right) \oplus H^{0}\left(R^{1} \pi_{*} V\right) .
$$


On the other hand, $H^{1}\left(R^{1} \pi_{*} \Lambda^{2} V\right)$ injects into $H^{2}\left(\Lambda^{2} V\right)$, so clearly $H^{1}\left(i_{\left\{a_{2}=0\right\} *}\left(\mathcal{L}_{1} \otimes \mathcal{L}_{2} \otimes\right.\right.$ $\left.K_{B}^{-1}\right)$ ) can contribute in $H^{2}\left(\Lambda^{2} V\right) .{ }^{11}$ After this point it is hard to say general statements about the Yukawa couplings, and the situation can defer case by case, depending on the morphisms between the cohomology groups. Here we only give the candidates for the Yukawa couplings, and after this point computing the actual couplings in a given model is usually simple.

Therefore we conclude that if $V$ is a stable, degree zero, holomorphic $\mathrm{SU}(3)$ bundle, with reducible spectral cover (but reduced components), then the 27-27-27 Yukawa coupling can be non-zero, and is coming from the interaction of zero modes localized over $\left\{a_{2}=0\right\}$ and bulk zero modes, and they correspond to the following morphism,

$$
H^{1}\left(\mathcal{L}_{1} \otimes K_{B}^{-1}\right) \otimes H^{0}\left(i_{\left\{a_{2}=0\right\} *} \mathcal{L}_{2}\right) \longrightarrow H^{1}\left(i_{\left\{a_{2}=0\right\} *}\left(\mathcal{L}_{1} \otimes \mathcal{L}_{2} \otimes K_{B}^{-1}\right)\right)
$$

F-theory interpretation of this result is again easy. This type of Yukawa coupling is simply coming from the bulk and localized zero modes, see figure 4. Note that the singularity of the F-theory Calabi-Yau fourfold corresponds to $E_{7}$. So one may naively expect to get the $E_{7}$ gauge group in the effective theory. But in the point is that the rank of the Higgs field over the GUT 7-brane is dropped by one (T-brane setup) [51, 52]

$$
\Phi=\left(\begin{array}{ccc}
0 & a_{2} & 0 \\
-\frac{1}{a_{0}} & 0 & 0 \\
0 & -\frac{1}{a_{0}} & 0
\end{array}\right)
$$

Therefore there will be a U(1) gauge field living over the complex surface that 7-brane wraps (isomorphic to the base of $X$ ), and that breaks $E_{7}$ to $E_{6}$. Hence the cohomology $H^{1}\left(\mathcal{L}_{1} \otimes K_{B}^{-1}\right)$ can be identified with the bulk zero modes inside the 7-brane (associated to the fluctuations of the $\mathrm{U}(1)$ connection), and $H^{0}\left(i_{\left\{a_{2}=0\right\}} \mathcal{L}_{2}\right)$ is identified with the zero modes living over the intersection of the GUT 7-brane and a flavor 7-brane. Of course, there are moduli dependent Yukawa couplings too:

$$
\operatorname{Ext}_{X}^{1}\left(\mathcal{O}_{X}, V\right) \otimes \operatorname{Ext}_{X}^{1}(V, V) \otimes \operatorname{Ext}_{X}^{1}\left(V, \mathcal{O}_{X}\right) \longrightarrow H^{3}\left(O_{X}\right) \simeq \mathbb{C} .
$$

Such morphisms ${ }^{12}$ correspond to $\mathbf{1 - 2 7 - 2 7}$ couplings in the effective theory. The F-theory interpretation of this is well known [90]. To connect this to F-theory, we rewrite the above product in terms of spectral sheaves

$$
\operatorname{Ext}^{1}\left(i_{\sigma *} \mathcal{O}, \mathcal{L}_{3}\right) \otimes \operatorname{Ext}^{1}\left(\mathcal{L}_{3}, \mathcal{L}_{3}\right) \otimes \operatorname{Ext}^{1}\left(\mathcal{L}_{3}, i_{\sigma *} \mathcal{O}\right) \longrightarrow \mathbb{C} .
$$

The first and the third term are $\mathbf{2 7}$ and $\overline{\mathbf{2 7}}$ as usual, and the middle term corresponds to the first order deformations of the spectral data (or the bundle $V$ ), and "can" receive contributions from $\operatorname{Ext} t^{1}\left(\mathcal{L}_{1}, \mathcal{L}_{3}\right)$. This later group can be interpreted as the open strings

\footnotetext{
${ }^{11}$ Remember that

$$
R \pi_{*} \Lambda^{2} V=L i_{\sigma}^{*} \mathcal{L}_{3} \star_{A} \mathcal{L}_{3} \otimes K_{B}[-1]
$$

${ }^{12}$ This is a result of Yoneda product.
} 


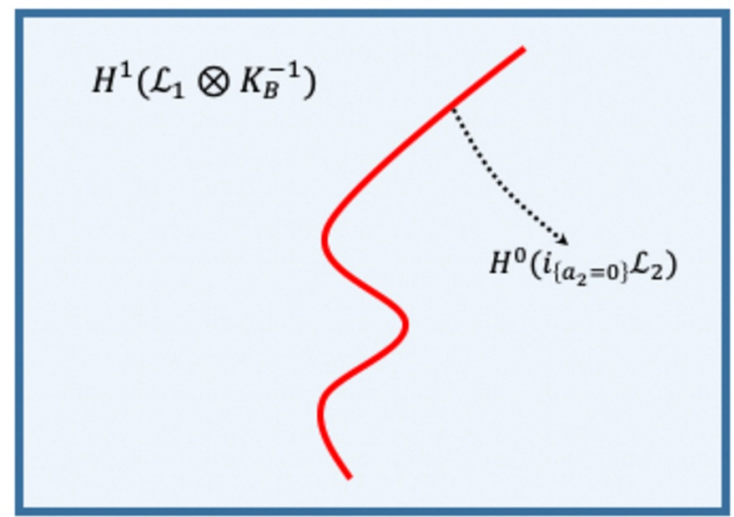

Figure 4. Some of $\mathbf{2 7}$ matter in the effective theory are from the bulk zero modes, and some of them are zero modes on the intersection of the 7-branes (red curve).

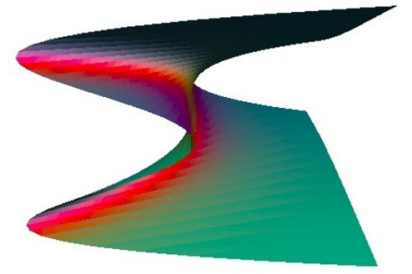

Figure 5. Smooth degree four cover of the base.

that are stretched between the components of the spectral cover (which in the local Ftheory correspond to components of intersecting 7-branes). We will see more details about this in examples.

\section{$3.2 \mathrm{SO}(10)$ GUT models}

For $\mathrm{SO}(10)$ models, we need $\mathrm{SU}(4)$ vector bundles, $V$ over the Calabi-Yau manifold $X$. Hence the spectral cover is a degree four cover of the base manifold. The number of the possibilities for spectral covers without vertical components is larger relative to the $E_{6}$ models.

\subsubsection{Smooth spectral cover}

In this case $V$ is a $\mathrm{SU}(4)$ vector bundle, and the spectral cover is a generic degree four smooth cover of the base,

$$
S_{4}=a_{4} X^{2}+a_{3} Y Z+a_{2} X Z^{2}+a_{0} Z^{4}
$$


As usual, $R^{1} \pi_{*} V$ receives a contribution from the matter curve $c:=a_{4}=0 .{ }^{13}$ So the "matter curve" for $\Lambda^{2} V$ is given by the non-reduced curve $a_{3}^{2}=0$. The reason (besides the reason mentioned in the footnote) can be explained as in the smooth degree three spectral cover. Suppose $S_{4}$ intersect a generic fiber at 4 points $P_{1}, P_{2}, P_{3}$ and $P_{4}$ such that

$$
P_{1} \boxplus P_{2} \boxplus P_{3} \boxplus P_{4}=0 .
$$

Then $S_{\Lambda^{2} V}$ intersect the same fiber at six points

$$
\begin{array}{lll}
P_{1} \boxplus P_{2}, & P_{1} \boxplus P_{3}, & P_{1} \boxplus P_{4}, \\
P_{2} \boxplus P_{3}, & P_{2} \boxplus P_{4}, & P_{3} \boxplus P_{4} .
\end{array}
$$

So when the zero section intersects with $S_{\Lambda^{2} V}$ (which corresponds to the locus of $\mathbf{1 0}$ of $\mathrm{SO}(10)$ ), one of these six points (say $P_{1} \boxplus P_{2}$ ) becomes zero, and due to (3.34), $P_{3} \boxplus P_{4}$ will be zero too. So the matter curve of $\Lambda^{2} V$ is the locus where not only (3.34) is true, but also the sum of a pair of these four points is zero. The algebraic equation for such points on the fiber must involve only $X$ and $Z$. The reason is $X$ is a degree two variable, and any holomorphic equation that involves $X$ gives a pair of points such that their sum is zero. This happens when $a_{3}=0$ in $S_{4}$. In other words

$$
\left.S_{4}\right|_{a_{3}=0}=a_{4} X^{2}+a_{2} X Z^{2}+a_{0} Z^{4},
$$

which is a degree four cover of $a_{3}=0$, or equivalently, a double (degree two) cover of the non-reduced curve $a_{3}^{2}=0$ (see figure 6),

$$
P:\left.S_{4}\right|_{a_{3}=0} \stackrel{2: 1}{\rightarrow} c_{\Lambda^{2} V}=a_{3}^{2}=0 .
$$

So as in the $\mathrm{SU}(3)$ bundle one gets the following relation,

$$
R \pi_{*} \Lambda^{2} V=\operatorname{Det}\left[\left.P_{*} \mathcal{L}\right|_{a_{3}=0}\right] \otimes K_{B}^{-1}[-1] .
$$

Note that $R^{1} \pi_{*} \Lambda^{2} V$ is non-zero over $a_{3}=0$, and $R^{1} \pi_{*} V$ is non-zero over the matter curve $a_{4}=0$, and their sections (i.e., the $H^{0}$ cohomology) inject into $H^{1}\left(\Lambda^{2} V\right.$ ) and $H^{1}(V)$ respectively. So the following map is non-zero

$$
H^{0}\left(R^{1} \pi_{*} V\right) \otimes H^{0}\left(R^{1} \pi_{*} V\right) \otimes H^{0}\left(R^{1} \pi_{*} \Lambda^{2} V\right) \longrightarrow H^{0}\left(p, \mathcal{O}_{p}\right),
$$

where $p$ corresponds to the intersection locus of $c$ and $c_{\Lambda^{2} V}$ in the base. Then the Yukawa coupling 16-16-10 is non-vanishing over the intersection locus of the matter curves.

The F-theory interpretation of this is again straightforward. The relevant part of the dual F-theory geometry (which can be derived by stable degeneration [88]) is given by

$$
\begin{aligned}
Y^{2}+X^{3}+\alpha_{1} v X Y & +\left(\alpha_{2} v^{2}+a_{4} u v\right) X^{2}+\left(\alpha_{2} v^{3}+a_{3} u v^{2}\right) Y \\
& +\left(\alpha_{4} v^{4}+a_{2} u v^{3}\right) X+\left(\alpha_{6} v^{6}+a_{0} u v^{5}\right)
\end{aligned}
$$

\footnotetext{
${ }^{13}$ It is also easy to show that the spectral cover of $\Lambda^{2} V$ (at least locally in the neighbourhood of $Z=0$ ) is given by the following equation,

$$
S_{\Lambda^{2} V}=-a_{3}^{2} X^{3}+\left(a_{2}^{2}-4 a_{0} a_{4}\right) X^{2} Z^{2}+a_{0}^{2} Z^{6} .
$$




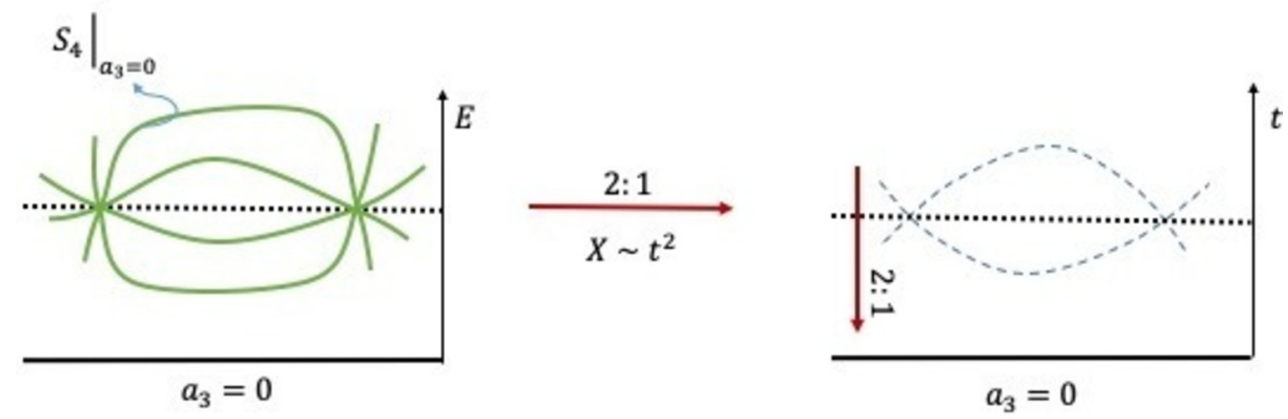

Figure 6. The green curve in the left picture is the restriction of $S_{4}$ over $a_{3}=0$. It is s degree 4 cover of the curve $a_{3}=0$ in the base manifold. Therefore if the two outer sheets correspond to $P_{1}$ and $P_{2}$ on a fiber such that $P_{1} \boxplus P_{2}=0$, then the inner sheets correspond to the points $P_{3}$ and $P_{4}$. By locally setting $X \sim \sqrt{t}$ it can be seen as the double cover of $a_{3}^{2}=0$.
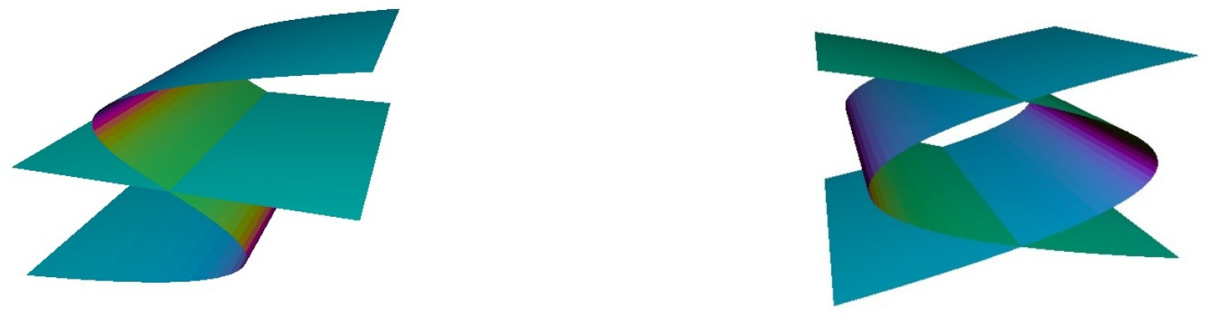

Figure 7. Reducible degree four spectral covers with reduced components.

Over $a_{4}=0$, the singularity enhances to $E_{6}$, so both $\mathbf{1 6}$ and $\overline{\mathbf{1 6}}$ lives in there, and over $a_{3}=0$, the singularity enhances to $\mathrm{SO}(11)$, which corresponds to the $\mathbf{1 0}$ hypermultiplets of $S(10)$ ("non-split" type singularity). At the intersection locus $a_{3}=a_{4}=0$, there are $E_{7}$ singularities enhancements which signal the original parent $E_{7}$ gauge theory that is broken to $\mathrm{SO}(10)$ due to brane deformation. In the original $E_{7}$ theory, there are triple interactions of the adjoint representation of $E_{7}$, and after the deformation it reduces to 16-16-10 coupling. Also on the branching points of the double cover in figure 6 , which is given by the discriminant $a_{2}^{2}-4 a_{0} a_{4}=0$, corresponds to the points where the singularity enhances to $\mathrm{SO}(14)$. From the heterotic string theory view, it happens because the restriction of the bundle over these branch points becomes like $\left(\mathcal{O}_{E}\left(P_{1}-\sigma\right) \oplus \mathcal{O}_{E}\left(-P_{1}-\sigma\right)\right)^{\oplus 2}$. This non-generic form of the bundle has a "larger" commutant in $E_{8}(\mathrm{SO}(14))$ relative to the direct sum of four generic degree zero line bundles. So the gauge group must enhance. However, these points don't give any Yukawa couplings in F-theory, as expected from the Heterotic view. 


\subsubsection{Reducible reduced spectral covers}

Let us continue to $\mathrm{SU}(4)$ bundles with reducible spectral covers such that each component is smooth, and there are no vertical components. In this case there are two possibilities (see figure 7),

$$
\begin{array}{ll}
S_{4}=Z\left(a_{3} Y+a_{2} X Z+a_{0} Z^{3}\right), & 0 \longrightarrow i_{\sigma *} \mathcal{L}_{1} \longrightarrow \mathcal{L}_{4} \longrightarrow i_{S_{3} *} \mathcal{L}_{3} \longrightarrow 0, \\
S_{4}=\left(a_{2} X+a_{0} Z^{2}\right)\left(b_{2} X+b_{0} Z^{2}\right), & 0 \longrightarrow i_{S_{2} *} \mathcal{L}_{2} \longrightarrow \mathcal{L}_{4} \longrightarrow i_{\tilde{S}_{2 *}} \mathcal{L}_{2}^{\prime} \longrightarrow 0,
\end{array}
$$

where $a_{i}$ and $b_{i}$ are generic polynomials.

- $S_{4}=Z\left(a_{3} Y+a_{2} X Z+a_{0} Z^{3}\right)$

For the first case the calculation is similar to the $\mathrm{SU}(3)$ bundle with reducible spectral cover. So generally the spectral sheaf $\mathcal{L}_{4}$, as an extension

$$
0 \longrightarrow \mathcal{L}_{1} \rightarrow \mathcal{L}_{4} \longrightarrow \mathcal{L}_{3} \longrightarrow 0
$$

The associated spectral sheaf for $\Lambda^{2} V$ corresponds to the antisymmetrized Pontrjagin product of $L_{4}$,

$$
0 \longrightarrow \pi^{*} \pi_{*} \mathcal{L}_{1} \otimes i_{S_{3} *} \mathcal{L}_{3} \longrightarrow \mathcal{L}_{4} \star_{A} \mathcal{L}_{4} \longrightarrow \mathcal{L}_{3} \star_{A} \mathcal{L}_{3} \longrightarrow 0
$$

It is possible to read the algebraic equation for the spectral cover of $\Lambda^{2} V$ from this sequence

$$
S_{\Lambda^{2} V}=\left(a_{3} Y+a_{2} X Z+a_{0} Z^{3}\right)\left(-a_{3} Y+a_{2} X Z+a_{0} Z^{3}\right),
$$

where the second factor is the support of $\mathcal{L}_{3} \star_{A} \mathcal{L}_{3}$. As before the left derived functor $L i_{\sigma}^{*}$ should be applied on the sequence above,

$$
\begin{gathered}
0 \longrightarrow L^{-1} i_{\sigma}^{*} \mathcal{L}_{4} \star_{A} \mathcal{L}_{4} \longrightarrow\left(\left.\operatorname{Det}_{*} \mathcal{L}_{3}\right|_{\tilde{c}_{m}}\right) \otimes K_{B}^{-1} \\
\left.\longrightarrow \mathcal{L}_{1} \otimes i_{c_{m}}^{*} \mathcal{L}_{3} \longrightarrow L^{0} i_{\sigma}^{*} \mathcal{L}_{4} \star_{A} \mathcal{L}_{4} \longrightarrow \operatorname{Det}_{*} \mathcal{L}_{3}\right|_{\tilde{c}_{m}} \longrightarrow
\end{gathered}
$$

where $\tilde{c}_{m}$ is the double cover defined in section 3.1.1,

$$
\pi: \tilde{c}_{m} \cup c_{m}=\left.S_{3}\right|_{\left\{a_{3}=0\right\}} \stackrel{3: 1}{\rightarrow} c_{m}=\left\{Z=a_{3}=0\right\} .
$$

As probably clear by now, for computing $H^{1}(V)$ through Leray spectral sequence, we need the derived pushforward of $V$

$$
\begin{aligned}
R \pi_{*} V & =\mathcal{L}_{1} \otimes K_{B}^{-1} \oplus \mathcal{F}[+1], \\
0 \longrightarrow \mathcal{L}_{1} & \left.\longrightarrow \mathcal{F} \longrightarrow \mathcal{L}_{3}\right|_{c} \longrightarrow 0 .
\end{aligned}
$$

As before, one should be careful to check whether the cohomologies of the pushforwards actually contribute in the relevant cohomologies of $V$ and $\Lambda^{2} V$. In the present case, as in the $\mathrm{SU}(3)$ bundle with reducible reduced spectral cover, the coboundary maps may not be zero because they can be induced by the extension group of the original sequence, which 
is the only defining parameter of this bundle (modulo the information needed to construct the constituent bundles).

Therefore, let $V$ be a SU(4) holomorphic stable bundle with degree zero with reducible spectral cover such that one component is zero section, and the other component a generic triple cover. Then there can be a non-vanishing 16-16-10 Yukawa coupling corresponding to the following map,

$$
H^{1}\left(\mathcal{L}_{1} \otimes K_{B}^{-1}\right) \otimes H^{0}\left(i_{\left\{a_{3}=0\right\} *} \mathcal{L}_{3}\right) \longrightarrow H^{1}\left(i_{\left\{a_{3}=0\right\} *}\left(\mathcal{L}_{1} \otimes \mathcal{L}_{3} \otimes K_{B}^{-1}\right)\right) .
$$

The F-theory interpretation is very similar to 3.1.2. So we ignore this case. We should just mention that as in the $\mathrm{SU}(3)$ bundle, there are moduli dependent Yukawa couplings 1-16-16, which has open string interpretation as before.

- $S_{4}=\left(a_{2} X+a_{0} Z^{2}\right)\left(b_{2} X+b_{0} Z^{2}\right)$

We proceed as usual. Consider the defining sequence,

$$
0 \longrightarrow \mathcal{L}_{2} \rightarrow \mathcal{L}_{4} \longrightarrow \mathcal{L}_{2}^{\prime} \longrightarrow 0 \text {. }
$$

Where $\mathcal{L}_{2}$ is supported over $S_{2}=a_{2} X+a_{0} Z^{2}$, and $\mathcal{L}_{2}^{\prime}$ is another line bundle supported over $S_{2}^{\prime}=b_{2} X+b_{0} Z^{2}$. The extension group of this sequence is given by

$$
\operatorname{Hom}_{S_{2} \cdot S_{2}^{\prime}}\left(\mathcal{L}_{2}^{\prime}, \mathcal{L}_{2} \otimes \mathcal{O}\left(S_{2}^{\prime}\right)\right)=H^{0}\left(\mathcal{L}_{2} \otimes \mathcal{L}_{2}^{*^{\prime}} \otimes \mathcal{O}\left(S_{2}\right)\right),
$$

where $S_{2} \cdot S_{2}^{\prime}$ is the intersection of the two double covers, and itself is a double cover of the curve,

$$
a_{2} b_{0}-a_{0} b_{2}=0 .
$$

As usual, the derived pushforward in this case is very simple to find

$$
R \pi_{*} V=L i_{\sigma}^{*} \mathcal{L}_{4}[-1]=i_{\sigma}^{*} \mathcal{L}_{4}[-1],\left.\left.0 \longrightarrow \mathcal{L}_{2}\right|_{c} \longrightarrow i_{\sigma}^{*} \mathcal{L}_{4} \longrightarrow \mathcal{L}_{2}^{\prime}\right|_{c^{\prime}} \longrightarrow 0,
$$

where $c$ and $c^{\prime}$ are curves given by $\left\{a_{2}=0\right\}$ and $\left\{b_{2}=0\right\}$ respectively. The matter curve is the union $c \cup c^{\prime}$. The extension of this sequence is induced by the extension of the original sequence. However, it can be zero, and it must be checked case by case. The next step is finding the antisymmetrized Pontrjagin product,

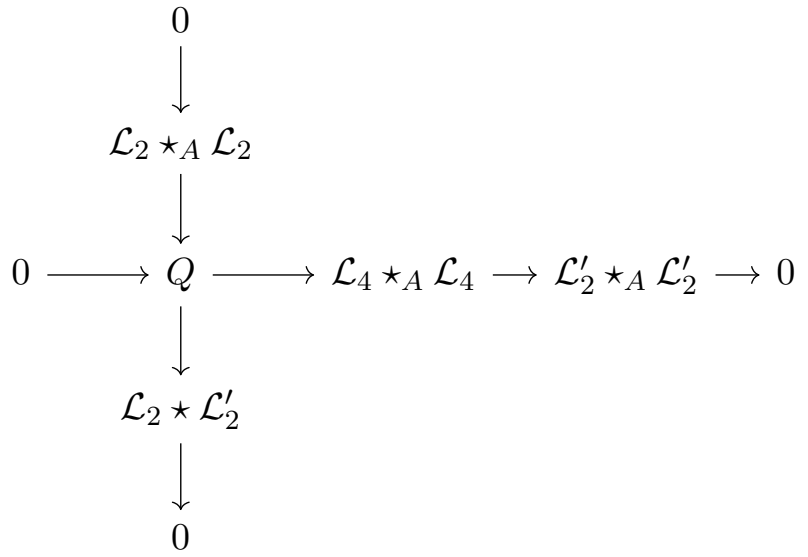


Either from this diagram or directly, we can find the spectral cover of $\Lambda^{2} V$ which is a degree six cover of the base $\mathrm{e}^{14}$

$$
S_{\Lambda^{2} V}=Z^{2}\left(\left(a_{2} b_{0}-a_{0} b_{2}\right)^{2} X^{2}+2 a_{0} b_{0}\left(a_{2} b_{0}+a_{0} b_{2}\right) X Z^{2}+a_{0}^{2} b_{0}^{2} Z^{4}\right) .
$$

The restriction over the zero section is

$$
\begin{aligned}
L i_{\sigma}^{*} \mathcal{L}_{4} \star_{A} \mathcal{L}_{4}= & \left(\operatorname{Det}\left(\pi_{*} \mathcal{L}_{2}\right) \oplus \operatorname{Det}\left(\pi_{*} \mathcal{L}_{2}^{\prime}\right) \oplus \pi_{*}\left(\mathcal{L}_{2} \otimes \tau^{*} \mathcal{L}_{2}^{\prime}\right)\right) \\
& \oplus\left(\operatorname{Det}\left(\pi_{*} \mathcal{L}_{2}\right) \oplus \operatorname{Det}\left(\pi_{*} \mathcal{L}_{2}^{\prime}\right)\right) \otimes K_{B}^{-1}[+1]
\end{aligned}
$$

Note that we expect the coboundary maps to be zero in this case. To find the relevant cohomologies we need to know the relations between the sheaves written above (see A)

$$
\operatorname{Det}\left(\pi_{*} \mathcal{L}_{2}\right) \otimes \operatorname{Det}\left(\pi_{*} \mathcal{L}_{2}^{\prime}\right)=K_{B}^{4}
$$

Unfortunately there are no 16-16-10 coupling from the interaction of bulk and localized zero modes. However there are Yukawa couplings from the triple intersection of 7-branes. The relevant cohomologies are

$$
H^{1}(V) \cap H^{1}(V) \cap H^{1}\left(\Lambda^{2} V\right) \longrightarrow H^{0}\left(\left.\mathcal{L}_{2}\right|_{c}\right) \cap H^{0}\left(\left.\mathcal{L}_{2}^{\prime}\right|_{c^{\prime}}\right) \cap H^{0}\left(\pi_{*}\left(\mathcal{L}_{2} \otimes \tau^{*} \mathcal{L}_{2}^{\prime}\right)\right) \longrightarrow \mathbb{C}
$$

So there are pointlike Yukawa couplings in this case. When the extension (3.50) is nonzero, it induces a non-zero morphisms on the intersection points of the matter curves of $V$. So, even though $H^{0}\left(\left.\mathcal{L}_{2}\right|_{c}\right)$ always injects into $H^{1}(V)$, but some or all of the elements of $H^{0}\left(\left.\mathcal{L}_{2}^{\prime}\right|_{c^{\prime}}\right)$ (especially when $H^{1}\left(\left.\mathcal{L}_{2}\right|_{c}\right)$ ) will be killed by the extension morphism. The physics of this is not very surprising, but interesting to know [94]. When we choose a zero extension, $V$ will be an $S(\mathrm{U}(2) \otimes \mathrm{U}(2)$ ) bundle (of course we should find a suitable point in Kahler moduli such that $V$ becomes poly stable). In this case, the effective gauge group will be $\mathrm{SO}(12) \times \mathrm{U}(1)$. The extra $\mathrm{U}(1)$ is generally anomalous and massive (for example see $[84,85])$. The hypermultiplets corresponding to $H^{0}\left(\left.\mathcal{L}_{2}\right|_{c}\right)$ and $H^{0}\left(\left.\mathcal{L}_{2}^{\prime}\right|_{c^{\prime}}\right)$ are in 32 and 32' representations of $S O(12)$, and the hypermultiplets corresponding to the extension morphism (3.50) are $\mathbf{1 2}$ of $\mathrm{SO}(12)$. On the other hand, $\mathrm{SO}(12)$ can break to $\mathrm{SO}(10) \otimes \mathrm{U}(1)$. So to Higgs $\mathrm{SO}(12)$ to $\mathrm{SO}(10)$ we need to give a vev to a hypermultiplet charged under the $\mathrm{U}(1)$ only. Note that under $\mathrm{SO}(12) \rightarrow \mathrm{SO}(10) \times \mathrm{U}(1)$, the $\mathbf{1 2}$ breaks as

$$
\mathbf{1 2} \rightarrow \mathbf{1}_{2} \oplus \mathbf{1}_{-2} \oplus \mathbf{1 0}_{0},
$$

\footnotetext{
${ }^{14}$ We compute the spectral cover of the associated bundles by simply adding the roots of the spectral cover (as in [87] for the Higgs bundle spectral cover). This is certainly true if we restrict $S_{V}$ to the neighbourhood of the zero section, and therefore can be used for the F-theory dual. But usually there is a caveat in finding the spectral cover of the associated bundles in heterotic side. The reason is that the fibers of the Calabi-Yau has torsion points relative to $\boxplus$ or the morphism $m$ (corresponding to the zeros of $Y$ ). These torsion points can slightly modify the spectral cover of the associated bundles in heterotic side. In the current example, over the points $a_{0} b_{2}-a_{2} b_{0}=a_{0}^{3}+F a_{0} a_{2}^{2}-G a_{2}^{3}=0$ (where $F, G$ are Weierstrass defining polynomials) the double covers $S_{2}$ and $S_{2}^{\prime}$ intersect the torsion points in the fibers, and these points are subset of their branching locus. So $S_{\Lambda^{2} V}$ too must be branched there, and this can checked to be true. The only feature that the author cannot explain are the vertical components over $a_{0}=b_{0}=0$. However they have to be there for correct divisor class.
} 
where the subscripts are the U(1) charges. Therefore by giving vev to $\mathbf{1}_{2}$ or $\mathbf{1}_{-2}$ breaks the gauge group to $\mathrm{SO}(10)$, and at the same time, the Yukawa couplings 12-32-32' gives mass to certain pairs of 32-32' multiplets. Compare this with the action of non-zero extension morphisms in either $\operatorname{Ext}^{1}\left(\mathcal{L}_{2}^{\prime}, \mathcal{L}_{2}\right)$ or $\operatorname{Ext} t^{1}\left(\mathcal{L}_{2}, \mathcal{L}_{2}^{\prime}\right)$. They also change the bundle from $S(\mathrm{U}(2) \times \mathrm{U}(2))$ to $\mathrm{SU}(4)$, and hence they break the $\mathrm{SO}(12)$ to $\mathrm{SO}(10)$. Also, the extension maps can kill (give mass) to some elements in $H^{0}\left(\mathcal{L}_{2}^{\prime}\right)$ (or $H^{0}\left(\mathcal{L}_{2}\right)$, depending on which extension morphism we choose). So we can identify the $\mathbf{1}_{2}$ and $1_{-2}$ hypermultiplets with elements in $\operatorname{Ext}^{1}\left(\mathcal{L}_{2}^{\prime}, \mathcal{L}_{2}\right)$ and $\operatorname{Ext}^{1}\left(\mathcal{L}_{2}, \mathcal{L}_{2}^{\prime}\right)$ (or vise versa, depending on the initial choice that we've made). Note that, when the extension is zero, in addition to (3.50) (which are part of the bundle moduli), there other hypermultiplets too, which are coming from $H^{1}\left(\Lambda^{2} V\right)$. These zero modes are also localized on $a_{2} b_{0}-a_{0} b_{2}$, and are given by $H^{0}\left(\pi_{*}\left(\mathcal{L}_{2} \otimes \tau^{*} \mathcal{L}_{2}^{\prime}\right)\right)$. These hypermultiplets also contribute to the 12-32-32' couplings. After turning on the extensions (3.50), or equivalently, Higgsing $\mathrm{SO}(12)$ to $\mathrm{SO}(10)$, then the 12s from $H^{1}\left(\Lambda^{2} V\right)$ will remain massless, and decompose into 10 of $\mathrm{SO}(10)$. Similarly, the corresponding Yukawa couplings decompose into 10-16-16 of the effective SO(10) Yukawa couplings, if the $\mathbf{3 2}$ and 32' hypermultiplets in those couplings remain massless after Higgsing (note that $\mathbf{3 2} \rightarrow \mathbf{1 6} \oplus \overline{\mathbf{1 6}}$ likewise for $\mathbf{3 2}$ ').

The dual F-theory interpretation is also quite similar. In F-theory, the geometry has split $I_{2}^{*}$ singularity (because the spectral cover is reducible). When the effective gauge group is really $\mathrm{SO}(12)$, one can check the singularity of the F-theory dual, and over the curves $c$ and $c^{\prime}$ inside the GUT 7-brane, enhances to $E_{7}$. The adjoint of the $E_{7}$ reduces to $\mathrm{SU}(2) \times \mathrm{SO}(12)$ as

$$
133 \rightarrow(3,1) \oplus\left(2,32^{\prime}\right) \oplus(1,66)
$$

So over these $E_{7}$ enhancements one gets only 32'. Where are the 12s and 32s? Note that $E_{7}$ enhancement means the parent gauge theory was $E_{7}$ and the brane deformation breaks this into $\mathrm{SO}(10)$. So lets "deform back" one of the components of the spectral cover that contains, say, $c$. Then the $E_{7}$ GUT singularity inside the 7-brane enhances to $E_{8}$ on the curve $c^{\prime}$. So over this curve the $\mathbf{5 6}$ hypermultiplet of $E_{7}$ lives. Now we deform the component that contained the curve $c$ to the initial generic one. Therefore the $\mathbf{5 6}$ also breaks according to the following rule

$$
56 \rightarrow(2,12) \oplus(1,32)
$$

where $(\mathbf{2}, \mathbf{1 2})$ corresponds to the strings that are charged relative to the gauge fields inside the GUT 7-brane. So after the deformation of the 7-brane these hypermultiplets will live on the intersection of the components of the spectral cover. Clearly 32 lives in the curve $c$. At this point, the rest of the stories is basically the same as the heterotic view, and we ignore the details. 


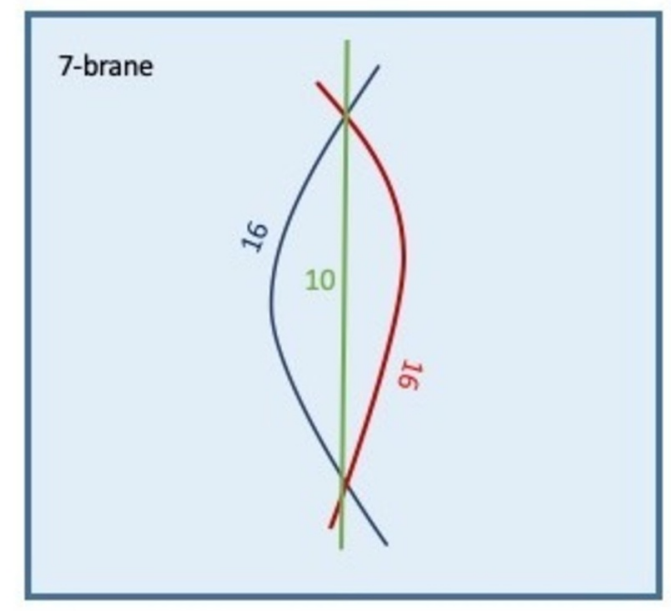

Figure 8. Yukawa couplings are coming from the intersection of the matter curves of $V$ and $\Lambda^{2} V$.
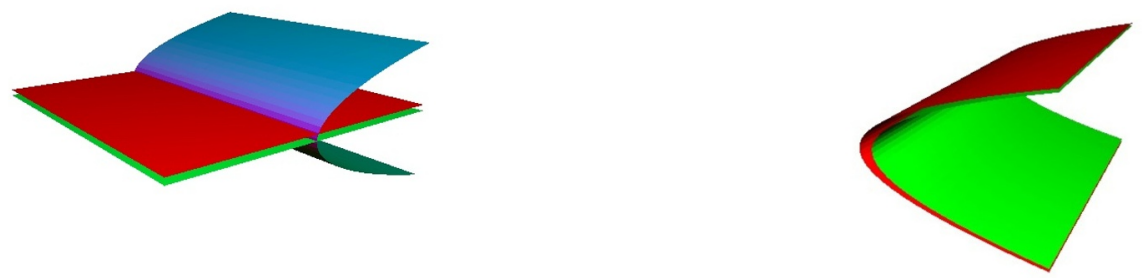

Figure 9. Non-reduced degree four spectral covers.

\subsubsection{Spectral covers with non-reduced components}

There are two cases that we are going to consider in this subsection. See figure 9. The spectral cover and the spectral sheaf in each case can be described generally as

$$
\begin{array}{ll}
S_{4}=Z^{2}\left(a_{2} X Z+a_{0} Z^{3}\right), & 0 \longrightarrow \mathcal{U}_{1} \longrightarrow \mathcal{L}_{4} \longrightarrow \mathcal{L}_{3} \longrightarrow 0, \\
S_{4}=\left(a_{2} X+a_{0} Z^{2}\right)^{2}, & 0 \longrightarrow \mathcal{L}_{2} \longrightarrow \mathcal{L}_{4} \longrightarrow \mathcal{L}_{2}^{\prime} \longrightarrow 0,
\end{array}
$$

We consider each case in turn.

- $\mathcal{L}_{4}$ is supported on $S_{4}=Z^{2}\left(a_{2} X+a_{0} Z^{2}\right)$.

We continue by iteration, and extend the result of 3.1.2

$$
0 \rightarrow \mathcal{U}_{1} \rightarrow \mathcal{L}_{4} \rightarrow \mathcal{L}_{3} \longrightarrow 0
$$

where $\mathcal{U}_{1}$ is supported on $\sigma$, and $\mathcal{L}_{3}$ is given by (3.16). First let us study the bundle $V$ 
itself and then continue to $\Lambda^{2} V$. The extension group can be derived by using dualising sheaf [82]

$$
\begin{aligned}
\operatorname{RHom}_{D(X)}\left(\mathcal{L}_{3}, \mathcal{U}_{1}\right) & =\operatorname{RHom}_{D(B)}\left(\operatorname{Li}_{\sigma}^{*} \mathcal{L}_{3}, \mathcal{U}_{1}\right) \\
& =\operatorname{RHom}_{D(B)}\left(\mathcal{L}_{1} \otimes K_{B}^{-1}[+1] \oplus \mathcal{F}, \mathcal{U}_{1}\right)
\end{aligned}
$$

where $\mathcal{F}$ is given in $(3.23)$,

$$
0 \longrightarrow \mathcal{L}_{1} \longrightarrow \mathcal{F} \longrightarrow i_{\left\{a_{2}=0\right\} *} \mathcal{L}_{2} \longrightarrow 0
$$

The equation gives a spectral sequence for $E x t^{1}$ group of the defining short exact sequence

$$
0 \longrightarrow \operatorname{Ext}^{1}\left(\mathcal{F}, \mathcal{U}_{1}\right) \longrightarrow \operatorname{Ext}^{1}\left(\mathcal{L}_{3}, \mathcal{U}_{1}\right) \longrightarrow \operatorname{Hom}\left(\mathcal{L}_{1} \otimes K_{B}^{-1}, \mathcal{U}_{1}\right) \longrightarrow \operatorname{Ext}^{2}\left(\mathcal{L}_{3}, \mathcal{U}_{1}\right)
$$

The first term on the left contains the gluing of the line bundle $\mathcal{U}_{1}$ and the line bundle over $S_{2}$ i.e., $\mathcal{L}_{2}$. It also contains a term $\left(\operatorname{Ext}^{1}\left(\mathcal{L}_{1}, \mathcal{U}_{1}\right)\right)$ that corresponds to making a rank 2 vector bundle over $\sigma$. This later part, if it contributes to $\operatorname{Ext}^{1}\left(\mathcal{L}_{3}, \mathcal{U}_{1}\right)$, should be kept zero. However, the term $\operatorname{Hom}\left(\mathcal{L}_{1} \otimes K_{B}^{-1}, \mathcal{U}_{1}\right)$, again if it contributes to $\operatorname{Ext}^{1}\left(\mathcal{L}_{3}, \mathcal{U}_{1}\right)$, corresponds to a "line bundle" over a degree two non-reduced surface, ad we need to keep in on generically (we denote a generic element of this group by $\mathcal{E} x t 4$ ). The next, task as usual, is computing the derived pushforward of $V$,

$$
\begin{aligned}
0 \longrightarrow & \mathcal{U}_{1} \otimes K_{B}^{-1} \longrightarrow \pi_{*} V \longrightarrow \mathcal{L}_{1} \otimes K_{B}^{-1}[+1] \\
& \longrightarrow \mathcal{U}_{1} \longrightarrow R^{1} \pi_{*} V \longrightarrow \mathcal{F} \longrightarrow
\end{aligned}
$$

The coboundary is given by the extension, therefore

$$
\begin{array}{r}
R \pi_{*} V=\mathcal{U}_{1} \otimes K_{B}^{-1} \oplus \overline{\mathcal{F}}[-1], \\
\left.0 \longrightarrow \mathcal{U}_{1}\right|_{\mathcal{E} x t 4=0} \longrightarrow \overline{\mathcal{F}} \longrightarrow \mathcal{F} \longrightarrow 0 .
\end{array}
$$

As usual, one needs the antisymmetrized Pontrjagin product

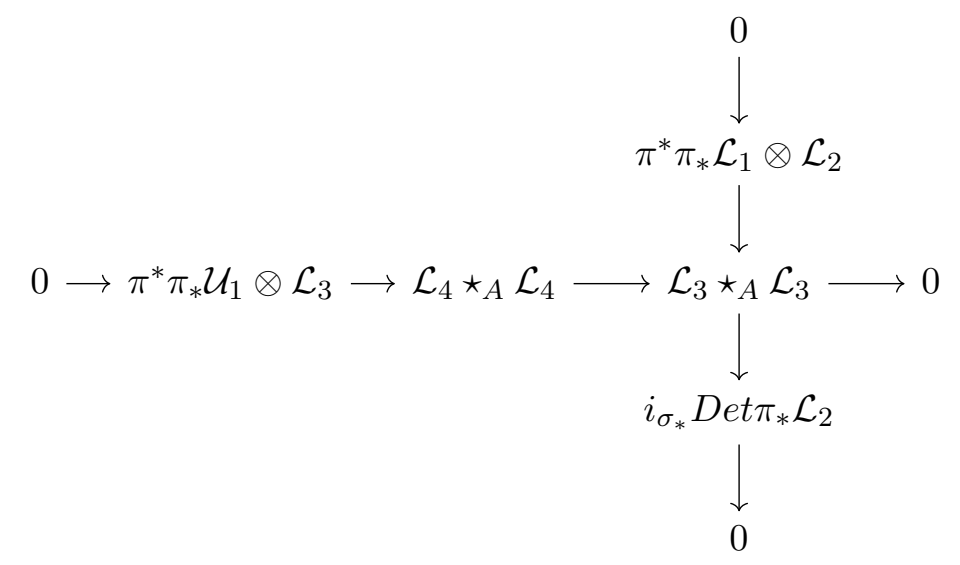


The extension of this sequence is generally non-zero. Before continuing, note that we can read the spectral cover of $\Lambda^{2} V$ from this sequence,

$$
S_{\Lambda^{2} V}=Z^{2}\left(a_{2} X+a_{0} Z^{2}\right)^{2}
$$

To compute $R^{1} \pi_{*} \Lambda^{2} V$, restrict $\mathcal{L}_{4} \star_{A} \mathcal{L}_{4}$ over $\sigma$

$$
\begin{aligned}
0 \longrightarrow \mathcal{L}_{1} \otimes \mathcal{U}_{1} \otimes K_{B}^{-1} \longrightarrow L^{-1} i_{\sigma}^{*} \mathcal{L}_{4} \star_{A} \mathcal{L}_{4} \longrightarrow L^{-1} i_{\sigma}^{*} \mathcal{L}_{3} \star_{A} \mathcal{L}_{3} \\
\\
\longrightarrow \mathcal{F} \otimes \mathcal{U}_{1} \longrightarrow L^{0} i_{\sigma}^{*} \mathcal{L}_{4} \star_{A} \mathcal{L}_{4} \longrightarrow i_{\sigma}^{*} \mathcal{L}_{3} \star_{A} \mathcal{L}_{3} \longrightarrow 0
\end{aligned}
$$

The coboundary map of this long exact sequence is induced by the extension of the $\mathcal{L}_{4} \star_{A} \mathcal{L}_{4}$ sequence. As in the previous sections, there is a 10-16-16 candidate

$$
H^{1}\left(\mathcal{U}_{1} \otimes K_{B}^{-1}\right) \otimes H^{0}(\mathcal{F}) \rightarrow H^{1}\left(\mathcal{U}_{1} \otimes \mathcal{F} \otimes K_{B}^{-1}\right)
$$

As usual, one should check whether these cohomology groups contribute to $H^{1}(V)$ and $H^{1}\left(\Lambda^{2} V\right)$ or not.

We should also mention that since $\mathcal{L}_{4}$ is defined by extending $\mathcal{L}_{3}$ with $\mathcal{U}_{1}$, one may expect the Yukawa couplings of the $\mathcal{L}_{3}$ should somehow contribute to the Yukawas for the SU(4) bundle we constructed. However, note that the Yukawa couplings of the SU(3) bundle depends on the zero modes $H^{1}\left(\mathcal{L}_{1} \otimes K_{B}^{-1}\right)$. But these modes are killed by the extension morphism of the line bundles on the two copies of the zero section (the $Z^{2}$ part of $\left.S_{4}\right)$ i.e., $\operatorname{Hom}\left(\mathcal{L}_{1} \otimes K_{B}^{-1}, \mathcal{U}_{1}\right)$. So if one restricts to non-trivial spectral sheaf with a non-reduced component $Z^{2}$, the Yukawa couplings of $\mathcal{L}_{3}$ will not contribute.

The F-theory dual is quite similar to the $\mathrm{SU}(3)$ bundle of 3.1.2. The only difference is that the flux that lives inside the 7-brane bulk is $\mathrm{SU}(2)$. So we ignore the detailed analysis.

- $S_{4}=S_{2}^{2}=\left(a_{2} X+a_{0} Z^{2}\right)^{2}$

The defining sequence is given by

$$
0 \rightarrow \mathcal{L}_{2} \rightarrow \mathcal{L}_{4} \rightarrow \mathcal{L}_{2}^{\prime} \longrightarrow 0
$$

where $\mathcal{L}_{2}$ and $\mathcal{L}_{2}^{\prime}$ are two different line bundles supported over the same double cover $S_{2}=a_{2} X+a_{0} Z^{2}$. The extension of this short exact sequence, should be chosen such that $\mathcal{L}_{4}$ corresponds to a "line bundle" (or numerically rank one to be precise) over the nonreduced surface $S_{4}$. The extension can be derived as usual by using the dualising sequence

$$
\operatorname{RHom}_{X}\left(\mathcal{L}_{2}^{\prime}, \mathcal{L}_{2}\right)=\operatorname{RHom}_{S_{2}}\left(\mathcal{L}_{2}^{\prime}, \mathcal{L}_{2} \otimes\left(\mathcal{O} \oplus \mathcal{O}_{S_{2}}\left(S_{2}\right)[-1]\right)\right) .
$$

Therefore one gets a spectral sequence corresponding to this

$$
0 \longrightarrow \operatorname{Ext}_{S_{2}}^{1}\left(\mathcal{L}_{2}^{\prime}, \mathcal{L}_{2}\right) \longrightarrow \operatorname{Ext}_{X}^{1}\left(\mathcal{L}_{2}^{\prime}, \mathcal{L}_{2}\right) \longrightarrow \operatorname{Hom}_{S_{2}}\left(\mathcal{L}_{2}^{\prime}, \mathcal{L}_{2} \otimes \mathcal{O}\left(S_{2}\right)\right) \stackrel{\alpha}{\longrightarrow} \operatorname{Ext}_{S_{2}}^{2}\left(\mathcal{L}_{2}^{\prime}, \mathcal{L}_{2}\right) \ldots
$$

So the first term on the left gives a rank 2 bundle over $S_{2}$ and this is not a spectral sheaf. "However, the elements of the third term, if are in the kernel of $\alpha$, correspond to 
a numerically rank one sheaf over the non-reduced surface $S_{4}$. For finding $H^{*}(V)$ we need the derived pushforward. It since $S_{4}$ intersects transversely with the zero section, only the first derived pushforward is non-zero

$$
\begin{gathered}
R^{1} \pi_{*} V=i_{\sigma}^{*} \mathcal{L}_{4} \Rightarrow H^{i}(V)=H^{i-1}\left(i_{\sigma}^{*} \mathcal{L}_{4}\right), \\
\left.\left.0 \longrightarrow \mathcal{L}_{2}\right|_{c} \longrightarrow i_{\sigma}^{*} \mathcal{L}_{4} \longrightarrow \mathcal{L}_{2}^{\prime}\right|_{c} \longrightarrow 0
\end{gathered}
$$

Note that the extension morphism of this sequence is induced by the extension group of the original sequence. The antisymmetrized Pontrjagin product can be computed by the following diagram

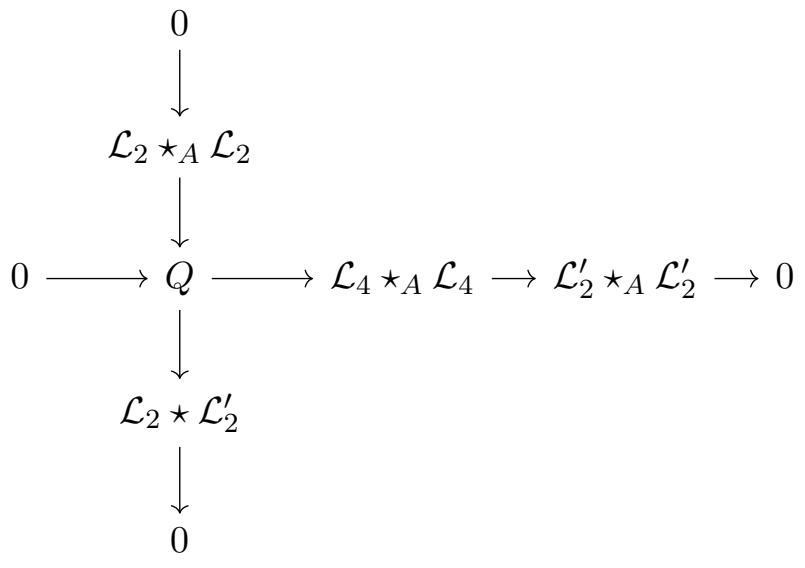

As in the double cover calculations, $\mathcal{L}_{2} \star_{A} \mathcal{L}_{2}=i_{\sigma *} \operatorname{Det}\left(\pi_{*} \mathcal{L}_{2}\right)$ and $\mathcal{L}_{2}^{\prime} \star_{A} \mathcal{L}_{2}^{\prime}=i_{\sigma *} \operatorname{Det}\left(\pi_{*} \mathcal{L}_{2}^{\prime}\right)$, so they are supported over the zero section. On the other hand, since the support of $\mathcal{L}_{2}$ and $\mathcal{L}_{2}^{\prime}$ are the same, the support of $\mathcal{L}_{2} \star_{A} \mathcal{L}_{2}^{\prime}$ should be reducible as $Z^{2} S_{2}^{\prime}$. So the spectral cover of $\Lambda^{2} V$ can be computed as (3.54)

$$
S_{\Lambda^{2} V}=Z^{4}\left(4 c_{t o r} a_{2} X+a_{0} Z^{2}\right),
$$

where $c_{t o r}$ is the part of the branching locus of the double cover $S_{2}$ which corresponds to the torsion points of the elliptic fibration. To find $R^{1} \pi_{*} \Lambda^{2} V$, we restrict $\mathcal{L}_{4} \star_{A} \mathcal{L}_{4}$ to the zero section,

$$
\begin{aligned}
L i_{\sigma}^{*} \mathcal{L}_{4} \star_{A} \mathcal{L}_{4}= & \operatorname{Det}_{*} \mathcal{L}_{2}^{\prime} \oplus \operatorname{Det}_{*} \mathcal{L}_{2} \oplus \Gamma\left(\mathcal{L}_{2}, \mathcal{L}_{2}^{\prime}\right) \\
& \oplus\left(\operatorname{Det}_{*} \mathcal{L}_{2}^{\prime} \oplus \operatorname{Det}_{*} \mathcal{L}_{2}\right) \otimes K_{B}^{-1}[+1] \oplus \bar{\Gamma}\left(\mathcal{L}_{2}, \mathcal{L}_{2}^{\prime}\right)[+1]
\end{aligned}
$$

where $\Gamma\left(\mathcal{L}_{2}, \mathcal{L}_{2}^{\prime}\right)$ and $\bar{\Gamma}\left(\mathcal{L}_{2}, \mathcal{L}_{2}^{\prime}\right)$ are a rank two bundles on $B$, derived from $\mathcal{L}_{2} \star_{A} \mathcal{L}_{2}^{\prime}$ part. We will derive them in the examples. The details of $\Gamma$ and $\bar{\Gamma}$ are necessary to find the Yukawa couplings.

In case the line bundles $\mathcal{L}_{2}$ and $\mathcal{L}_{2}^{\prime}$ can be written as $\mathcal{O}_{S_{2}}\left(\sigma+D_{1}\right)$ and $\mathcal{O}_{S_{2}}\left(\sigma+D_{2}\right)$ respectively, there are Yukawa couplings from the bulk and localized zero modes interactions. We leave the details for the examples (the SU(5) bundle example). First remember that

$$
\begin{aligned}
c_{1}\left(\pi_{*} \mathcal{L}_{4}\right) & =c_{1}\left(\pi_{*} \mathcal{L}_{2}\right)+c_{1}\left(\pi_{*} \mathcal{L}_{2}^{\prime}\right)=4 c_{1}\left(K_{B}\right), \\
& \Rightarrow-\eta+D_{1}+D_{2}=c_{1}\left(K_{B}\right),
\end{aligned}
$$


where the divisor class of the double cover is $\left[S_{2}\right]=2 \sigma+\eta$. Also we can compute the derived pushforwards of $\Lambda^{2} V$,

$$
\begin{aligned}
R \pi_{*} \Lambda^{2} V= & {\left[\mathcal{O} \oplus \mathcal{O}\left(K_{B}-2 D_{1}-2 D_{2}\right) \oplus \mathcal{O}\left(-\eta+2 D_{1}\right) \oplus \mathcal{O}\left(-\eta+2 D_{2}\right)\right] } \\
& \oplus\left[K_{B} \oplus \mathcal{O}\left(2 D_{1}+2 D_{2}\right) \oplus \mathcal{O}\left(K_{B}-\eta+2 D_{1}\right) \oplus \mathcal{O}\left(K_{B}-\eta+2 D_{2}\right)\right][-1] .
\end{aligned}
$$

With these information (also remember $K_{c}=K_{B}+[c]=3 K_{B}+\eta$ ) it is possible find the possible Yukawa couplings (modulo the coboundary maps as usual)

$$
\begin{aligned}
H^{0}\left(\mathcal{O}_{c}\left(K_{B}+D_{1}\right)\right) \otimes H^{0}\left(\mathcal{O}_{c}\left(K_{B}+D_{1}\right)\right) \otimes H^{1}\left(\mathcal{O}\left(-\eta+2 D_{2}\right)\right) & \longrightarrow H^{1}\left(\mathcal{O}_{c}\left(K_{c}\right)\right) \simeq \mathbb{C} \\
H^{0}\left(\mathcal{O}_{c}\left(K_{B}+D_{2}\right)\right) \otimes H^{0}\left(\mathcal{O}_{c}\left(K_{B}+D_{2}\right)\right) \otimes H^{1}\left(\mathcal{O}\left(-\eta+2 D_{1}\right)\right) & \longrightarrow H^{1}\left(\mathcal{O}_{c}\left(K_{c}\right)\right) \simeq \mathbb{C}, \\
H^{0}\left(\mathcal{O}_{c}\left(K_{B}+D_{1}\right)\right) \otimes H^{0}\left(\mathcal{O}_{c}\left(K_{B}+D_{2}\right)\right) \otimes H^{1}(\mathcal{O}) & \longrightarrow H^{1}\left(\mathcal{O}_{c}\left(K_{c}\right)\right) \simeq \mathbb{C} .
\end{aligned}
$$

F-theory description of this is very similar to the reducible (but reduced) spectral cover $S_{4}=S_{2} S_{2}^{\prime}$. So we comment on the F-theory dual briefly. The F-theory geometry has a split singularity $I_{2}^{* s}$ over the GUT 7-brane, which corresponds to a $\mathrm{SO}(12)$ effective gauge group. This would be the case if the extension morphism of the defining short exact sequence were chosen to be zero. The first difference relative to $S_{4}=S_{2} S_{2}^{\prime}$ case is that over the curve $c$ the singularity enhances to $E_{8}$. We can see the adjoint of the of $E_{8}$ decomposes as

$$
\begin{aligned}
E_{8} & \longrightarrow \mathrm{SU}(2) \times \mathrm{SU}(2) \times \mathrm{SO}(12), \\
248 & \longrightarrow(\mathbf{3}, \mathbf{1}, \mathbf{1}) \oplus(\mathbf{1}, \mathbf{3}, \mathbf{1}) \oplus\left(\mathbf{1}, \mathbf{2}, \mathbf{3 2} \mathbf{2}^{\prime}\right) \oplus(\mathbf{1}, \mathbf{1}, \mathbf{6 6}) \oplus(\mathbf{2}, \mathbf{2}, \mathbf{1 2}) \oplus(\mathbf{2}, \mathbf{1}, \mathbf{3 2}) .
\end{aligned}
$$

The second difference is that the 12s that are responsible for Higgsing $\mathrm{SO}(12)$ to $\mathrm{SO}(10)$ (or equivalently the extension maps) are non-localized inside the 7-brane. Geometrically they correspond to the deformations of $S_{2}$ (equivalently deformations of the 7-branes). So after Higgsing the remaining Yukawa couplings 12-32-32' (12s from $\left.H^{1}\left(\Lambda^{2} V\right)\right)$ decompose to 10-16-16. The third difference is that in this example the $\mathbf{1 6} \mathrm{s}$ are localize over the 7-brane, and 10s live in the bulk.

\section{$3.3 \quad \mathrm{SU}(5)$ model}

We conclude this section with $\mathrm{SU}(5)$ models, which can be constructed by putting a $\mathrm{SU}(5)$ vector bundle $V$ over the Calabi-Yau $X$. The strategy is the same as before. Simply rewrite the spectral data as an extension of line bundles on different components, and use the results have been derived for lower degree spectral covers in the previous sections.

The new feature of SU(5) models is that there are two possible Yukawa couplings, 10-10-5 and 10-5-5, where the first one corresponds to the usual map,

$$
H^{1}(V) \otimes H^{1}(V) \otimes H^{1}\left(\Lambda^{3} V\right) \longrightarrow \mathbb{C} \quad \Longrightarrow \quad H^{1}(V) \otimes H^{1}(V) \longrightarrow H^{2}\left(\Lambda^{2} V\right)
$$

while the second Yukawa coupling corresponds to

$$
H^{1}(V) \otimes H^{1}\left(\Lambda^{2} V\right) \otimes H^{1}\left(\Lambda^{2} V\right) \longrightarrow \mathbb{C}
$$




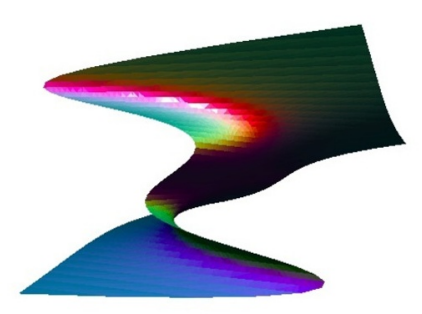

Figure 10. Smooth degree five spectral cover.

\subsubsection{Smooth spectral cover}

As always, the starting point is the generic spectral cover, figure 10. We start with computing the pushforward $R^{1} \pi_{*} \Lambda^{2} V$ for a smooth generic spectral cover of $\mathrm{SU}(5)$ bundle,

$$
S_{5}=a_{5} X Y+a_{4} X^{2} Z+a_{3} Y Z^{2}+a_{2} X Z^{3}+a_{0} Z^{5} .
$$

Consider a generic fiber, and the spectral cover intersect with it at five points $P_{1}, \ldots, P_{5}$ such that $P_{1} \boxplus \cdots \boxplus P_{5}=0$. So $S_{\Lambda^{2} V}$ intersect with the same fiber at the following ten points,

$$
P_{1} \boxplus P_{2}, \quad P_{1} \boxplus P_{3}, \ldots, \quad P_{4} \boxplus P_{5} .
$$

So the contribution to the $R^{1} \pi_{*} \Lambda^{2} V$ comes from a double cover. Similar to $\mathrm{SU}(3)$ and SU(4) bundles, if for example $P_{1} \boxplus P_{2}=0$ then $P_{3} \boxplus P_{4} \boxplus P_{5}=0$. So we should look for a locus where the spectral cover decomposes into a degree two and a degree three cover. In other words, the non-zero contribution is coming from $S_{5} \cap \tau\left(S_{5}\right)$,

$$
a_{5} X+a_{3} Z^{2}=0, \quad a_{4} X^{2}+a_{2} X Z^{2}++a_{0} Z^{4}=0,
$$

by eliminating $X$ in these equations we get a double cover $\tilde{c}$ over curve on base defined by

$$
\tilde{c}: \quad a_{5} X+a_{3} Z^{2}=0, \quad a_{4} a_{3}^{2}-a_{2} a_{3} a_{5}+a_{0} a_{5}^{2}=0 .
$$

So $R^{1} \pi_{*} \Lambda^{2} V$ is given by the formula (3.11), but the double cover $\tilde{c}$ defined as above.

Similar to the previous Yukawa couplings for smooth spectral covers. There are pointlike couplings (both 10-10-5 and $\overline{\mathbf{5}-5-10}$ ) coming from the intersection of the matter curve (or the $\mathbf{1 0}$ curve $c$ ) $a_{5}=0$ and the $\mathbf{5}$ curve (or $\overline{\mathbf{5}}$ curve) $a_{4} a_{3}^{2}-a_{2} a_{3} a_{5}+a_{0} a_{5}^{2}=0\left(c^{\prime}\right)$. The F-theory dual is quite simple. Inside the SU(5) GUT 7-brane the singularity enhances to $\mathrm{SO}(10)$ over $c$. This is not surprising since either from the heterotic view of local $\mathrm{F}$ theory Higgs bundle, the spectral cover reduces to $S_{4}$. Over this curve, the $\mathbf{1 0}$ and $\overline{\mathbf{1 0}}$ hypermultiplets live, as can be seen by the decomposition of $\mathbf{4 5}$ of $\mathrm{SO}(10)$,

$$
\begin{aligned}
\mathrm{SO}(10) & \longrightarrow \mathrm{SU}(5) \times \mathrm{U}(1), \\
\mathbf{4 5} & \longrightarrow \mathbf{1}_{0} \oplus \mathbf{1 0}_{4} \oplus \overline{\mathbf{1 0}}_{-4} \oplus \mathbf{2 4}_{0} .
\end{aligned}
$$



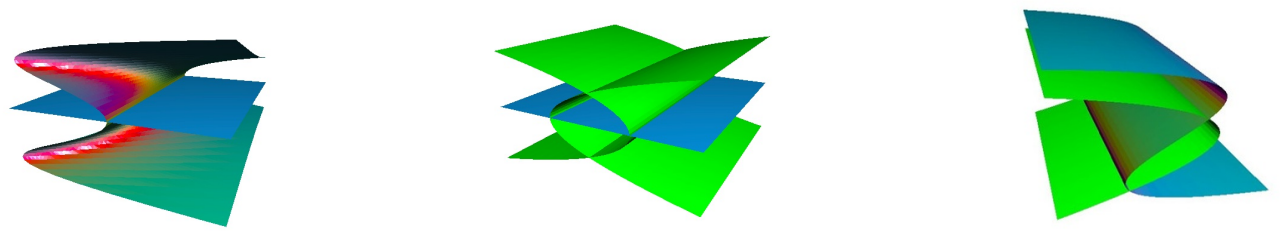

Figure 11. Reducible degree five spectral covers with smooth components.

Over the curve $c^{\prime}$, the singularity enhances by 1, i.e., the order of the discriminant jumps from 5 to 6 . However, the spectral cover becomes reducible (3.90), so the singularity of the F-theory geometry also will be of split type, i.e., $I_{6}^{s}$. So the singularity enhances to $\mathrm{SU}(6)$ over $c^{\prime}$. Again this is not unexpected from heterotic side since the bundle (ignoring the extension morphisms) becomes $S(\mathrm{U}(2) \times \mathrm{U}(3))$ over $c^{\prime}$, and its commutant in $E_{8}$ is $\mathrm{SU}(6) \times \mathrm{U}(1)$ (the extra $\mathrm{U}(1)$ is anomalous). By decomposing the $\mathbf{3 5}$ of $\mathrm{SU}(6)$ one can figure out that the $\mathbf{5}$ and $\overline{\mathbf{5}}$ hypermultiplets live over $c^{\prime}$

$$
\begin{aligned}
\mathrm{SU}(6) & \longrightarrow \mathrm{SU}(5) \times \mathrm{U}(1), \\
\mathbf{3 5} & \longrightarrow \mathbf{1}_{0} \oplus \mathbf{5}_{6} \oplus \overline{\mathbf{5}}_{-6} \oplus \mathbf{2 4 .}
\end{aligned}
$$

Hence we get the advertised Yukawa couplings overs the intersections of $c$ and $c^{\prime}$.

\subsubsection{Reducible reduced spectral covers}

In this case there are three possibilities (see figure 11),

$$
\begin{array}{lll}
S_{5}=Z\left(a_{4} X^{2}+a_{3} Y Z+a_{2} X Z^{2}+a_{0} Z^{4}\right), & \left.0 \longrightarrow \mathcal{W}_{1} \longrightarrow \mathcal{L}_{5} \longrightarrow \mathcal{L}_{4}\right|_{S_{4}} \longrightarrow 0, \\
S_{5}=Z\left(a_{2} X+a_{0} Z^{2}\right)\left(b_{2} X+b_{0} Z^{2}\right), & \left.0 \longrightarrow \mathcal{W}_{1} \longrightarrow \mathcal{L}_{5} \longrightarrow \mathcal{L}_{4}\right|_{S_{2} \tilde{S}_{2}} \longrightarrow 0, \\
S_{5}=S_{2} S_{3} & \left.\left.0 \longrightarrow \mathcal{L}_{2}\right|_{S_{2}} \longrightarrow \mathcal{L}_{5} \longrightarrow \mathcal{L}_{3}\right|_{S_{3}} \longrightarrow 0 .
\end{array}
$$

We study each case, by iteration as before.

- $S_{5}=Z\left(a_{4} X^{2}+a_{3} Y Z+a_{2} X Z^{2}+a_{0} Z^{4}\right)$

This case, i.e., a reducible spectral cover with one component being zero section and the other component being generic, should be easy by now. The dfining short exact sequence is

$$
\left.0 \longrightarrow \mathcal{W}_{1} \longrightarrow \mathcal{L}_{5} \longrightarrow \mathcal{L}_{4}\right|_{S_{4}} \longrightarrow 0
$$

The extension morphisms live inside the group

$$
\operatorname{Ext}^{1}\left(\mathcal{L}_{4}, \mathcal{W}_{1}\right)=H^{0}\left(c, \mathcal{W}_{1} \otimes \mathcal{L}_{4}^{*} \mathcal{O}_{c}(c)\right),
$$

where $c=S_{4} \cap \sigma=\left\{a_{4}=0\right\}$. To find $H^{1}(V)$ we need the derived pushforwards of $V$

$$
\begin{gathered}
R \pi_{*} V=\mathcal{W}_{1} \otimes K_{B}^{-1} \oplus \mathcal{F}[-1], \\
\left.0 \rightarrow \mathcal{W}_{1} \rightarrow \mathcal{F} \longrightarrow \mathcal{L}_{4}\right|_{a_{4}=0} \rightarrow 0
\end{gathered}
$$


For $R \pi_{*} \Lambda^{2} V$, again we use the antisymmetrized sequence,

$$
\left.0 \longrightarrow \pi_{1}^{*} \mathcal{W}_{1} \otimes \pi_{2}^{*} \mathcal{L}_{4}\right|_{S_{4}} \longrightarrow \pi_{1}^{*} \mathcal{L}_{5} \otimes_{A} \pi_{2}^{*} \mathcal{L}_{5} \longrightarrow \pi_{1}^{*} \mathcal{L}_{4} \otimes_{A} \pi_{4}^{*} \mathcal{L}_{2} \longrightarrow 0
$$

Hence,

$$
\left.0 \longrightarrow R^{0} m_{*} \pi_{1}^{*} \mathcal{W}_{1} \otimes \pi_{2}^{*} \mathcal{L}_{4}\right|_{S_{4}} \longrightarrow R^{0} m_{*} \pi_{1}^{*} \mathcal{L}_{5} \otimes_{A} \pi_{2}^{*} \mathcal{L}_{5} \longrightarrow R^{0} m_{*} \pi_{1}^{*} \mathcal{L}_{4} \otimes_{A} \pi_{2}^{*} \mathcal{L}_{4} \longrightarrow 0
$$

Acting on this with $L i_{\sigma}^{*}$,

$$
\left.\left.0 \longrightarrow\left(\mathcal{W}_{1} \otimes \mathcal{L}_{4}\right)\right|_{a_{4}=0} \longrightarrow i_{\sigma}^{*} \mathcal{L}_{5} \star_{A} \mathcal{L}_{5} \longrightarrow P_{*} \mathcal{L}_{4}\right|_{a_{3}^{2}=0} \longrightarrow 0
$$

where $P$ is the morphism defined in 3.2.1. As a byproduct, it is possible to read the spectral cover of $\Lambda^{2} V$ from this sequence

$$
S_{\Lambda^{2} V}=\left(-a_{3}^{2} X^{3}+\left(a_{2}^{2}-4 a_{0} a_{4}\right) X^{2} Z^{2}+a_{0}^{2} Z^{6}\right)\left(a_{4} X^{2}+a_{3} Y Z+a_{2} X Z^{2}+a_{0} Z^{4}\right) .
$$

So similar to the previous section there is a candidate for non-vanishing Yukawa coupling 10-10-5 when the following cohomologies contribute in $H^{1}(V)$ and $H^{2}\left(\Lambda^{2} V\right)$

$$
H^{1}\left(B, \mathcal{W}_{1} \otimes K_{B}^{-1}\right) \otimes H^{0}\left(\left.\mathcal{L}_{4}\right|_{a_{4}=0}\right) \longrightarrow H^{1}\left(\left.\left(\mathcal{W}_{1} \otimes \mathcal{L}_{4}\right)\right|_{a_{4}=0} \otimes K_{B}^{-1}\right)
$$

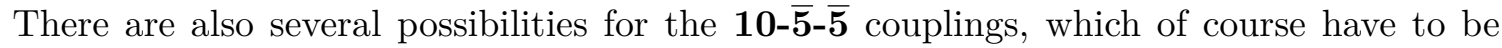
checked whether the following cohomologies inject into the $H^{1}(V)$ and $H^{1}\left(\Lambda^{2} V\right)$,

$$
\begin{aligned}
H^{0}\left(\mathcal{W}_{1}\right) \otimes H^{0}\left(\left.\left(\mathcal{W}_{1} \otimes \mathcal{L}_{4}\right)\right|_{a_{4}=0}\right) \otimes H^{0}\left(\left.P_{*} \mathcal{L}_{4}\right|_{a_{3}^{2}=0}\right) & \longrightarrow \mathbb{C}, \\
H^{0}\left(\left.\mathcal{L}_{4}\right|_{a_{4}=0}\right) \otimes H^{0}\left(\left.\left(\mathcal{W}_{1} \otimes \mathcal{L}_{4}\right)\right|_{a_{4}=0}\right) \otimes H^{0}\left(\left.P_{*} \mathcal{L}_{4}\right|_{a_{3}^{2}=0}\right) & \longrightarrow \mathbb{C}, \\
H^{0}\left(\left.\mathcal{L}_{4}\right|_{a_{4}=0}\right) \otimes H^{0}\left(\left.P_{*} \mathcal{L}_{4}\right|_{a_{3}^{2}=0}\right) \otimes H^{0}\left(\left.P_{*} \mathcal{L}_{4}\right|_{a_{3}^{2}=0}\right) & \longrightarrow \mathbb{C} .
\end{aligned}
$$

F-theory dual again is given in terms of T-branes like 3.1.2 . From the spectral data, it is clear that some of the 10s are living in the bulk of the $\mathrm{SO}(10)$ 7-brane, while some are in the $a_{4}=0$ curve. However, all of the $\mathbf{5}$ and $\overline{\mathbf{5}}$ live in either $a_{4}=0$ or $a_{4}=0$.

To see this in F-theory, first note that the Calabi-Yau's singularity is $\mathrm{SO}(10)$, this means there is a $\mathrm{U}(1)$ gauge group inside the 7 -brane (T-brane). In principle, this $\mathrm{U}(1)$ and the associated zero modes in the 7-brane bulk can be read from the spectral data or equivalently from the Higgs bundle hypercohomology [88]. However, this is outside of the scope of this paper. The singularity structure of the F-theory geometry is basically the same as smooth $S_{4}$. In other words there are enhancements to $\mathrm{SO}(11)$ and $E_{6}$ over $a_{3}=0$ and $a_{4}=0$ respectively. Purely from geometry one might say the gauge group in the effective theory is $\mathrm{SO}(10)$, and there are $\mathbf{1 0 s}$ of $\mathrm{SO}(10)$ in $a_{3}=0$ and $\mathbf{1 6}$ s and $\overline{\mathbf{1 6}} \mathrm{s}$ on $s_{4}=0$. In addition there is a gauge field in the bulk in the $\mathbf{4 5}$ (adjoint) of $\mathrm{SO}(10)$. Now consider the effect of the U(1) living in the bulk of the 7-brane. First of all the $\mathbf{4 5}$ breaks as

$$
\mathbf{4 5} \longrightarrow \mathbf{1}_{0} \oplus \mathbf{1 0}_{4} \oplus \overline{\mathbf{1 0}}_{-4} \oplus \mathbf{2 4}_{0}
$$


So one gets the bulk 10 s and $\overline{\mathbf{1 0}}$ s of the $\mathrm{SU}(5)$, identified with $H^{1}\left(\mathcal{W}_{1} \otimes K_{B}^{-1}\right)$. The $\mathbf{1 6}$ that lives in $a_{4}=0$ breaks as

$$
16 \longrightarrow 1_{-5} \oplus \overline{\mathbf{5}}_{3} \oplus \mathbf{1 0}_{-1} .
$$

Similar decomposition for $\overline{\mathbf{1 6}} \mathrm{s}$. So this is the source of the $\mathbf{5 s}$ and $\mathbf{1 0}$ s (and their conjugates) of SU(5) over $a_{4}=0$. Similarly, the $\mathbf{1 0}$ of $\mathrm{SO}(10)$ over $a_{3}=0$ break into $\mathbf{5}$ and $\overline{\mathbf{5}}$ of SU(5). So we see there are no $10 \mathrm{~s}$ or $\overline{\mathbf{1 0}} \mathrm{s}$ of $\mathrm{SU}(5)$ on $a_{3}=0$. In agreement with the heterotic calculations.

- $S_{5}=Z\left(a_{2} X+a_{0} Z^{2}\right)\left(b_{2} X+b_{0} Z^{2}\right)$

In this case there is 10-10-5 from $H^{1}(V) \otimes H^{1}(V) \otimes H^{1}\left(\Lambda^{3} V\right)$ in the usual way. There are $\overline{\mathbf{5}} \overline{\mathbf{5}} \mathbf{- 1 0}$ candidates from

$$
H^{0}\left(c_{\Lambda^{2} V_{4}}, \pi_{*}\left(\mathcal{L}_{2} \otimes \tau^{*} \mathcal{L}_{2}^{\prime}\right)\right) \otimes H^{0}\left(c,\left.\mathcal{W}_{1} \otimes \mathcal{L}_{2}\right|_{\sigma}\right) \otimes H^{0}\left(c^{\prime}, \mathcal{L}_{2}^{\prime}\right) \longrightarrow \mathbb{C}
$$

where the first two terms can contribute in $H^{1}\left(\Lambda^{2} V\right)$ and the third one contribute in $H^{1}(V)$. The curves $c_{\Lambda^{2} V_{4}}, c$ and $c^{\prime}$ are given by equations $a_{2} b_{0}-a_{0} b_{2}=0, a_{2}=0$ and $b_{2}=0$ respectively. One can also swap the $\mathcal{L}_{2}$ and $\mathcal{L}_{2}^{\prime}$ in the last two factors. To be complete let us add the details in this case too, take the defining sequence

$$
0 \longrightarrow \mathcal{W}_{1} \longrightarrow \mathcal{L}_{5} \longrightarrow \mathcal{L}_{4} \longrightarrow 0
$$

the atisymmetrized product can be found as usual,

$$
0 \longrightarrow \pi^{*} \pi_{*} \mathcal{W}_{1} \otimes \mathcal{L}_{4} \rightarrow \mathcal{L}_{5} \star_{A} \mathcal{L}_{5} \rightarrow \mathcal{L}_{4} \star_{A} \mathcal{L}_{4} \rightarrow 0
$$

where $\mathcal{L}_{4} \star_{A} \mathcal{L}_{4}$ is given in (3.53). Therefore the spectral cover is

$$
S_{\Lambda^{2} V}=Z^{2}\left(\left(a_{2} b_{0}-a_{0} b_{2}\right)^{2} X^{2}+2 a_{0} b_{0}\left(a_{2} b_{0}+a_{0} b_{2}\right) X Z^{2}+a_{0}^{2} b_{0}^{2} Z^{4}\right)\left(a_{2} X+a_{0} Z^{2}\right)\left(b_{2} X+b_{0} Z^{2}\right) .
$$

Therefore the 10-10-5 coupling is related to the following cohmologies,

$$
H^{0}\left(B, \mathcal{W}_{1} \otimes K_{B}^{-1}\right) \otimes H^{0}\left(c \cup c^{\prime},\left.\mathcal{L}_{4}\right|_{a_{2} b_{2}=0}\right) \longrightarrow H^{1}\left(\left.\pi^{*} \pi_{*} \mathcal{W}_{1} \otimes \mathcal{L}_{4}\right|_{a_{2} b_{2}=0} \otimes K_{B}^{-1}\right),
$$

which is coming from the bulk and localized zero modes.

The F-theory view is also similar to the previous case. Adding a copy of the zero section in the spectral cover is equivalent to a $5 \times 5$ Higgs filed with rank four inside the 7-brane, hence there will be q bulk gauge field living inside the surface the 7-brane wraps. This $\mathrm{U}(1)$ flux breaks the $\mathrm{SO}(10)$ further to $\mathrm{SU}(5)$ compatible with the heterotic view. Consequently, there will be $\mathbf{1 0}$ hypermultiplets of SU(5) identified with bulk zero modes $H^{1}\left(\mathcal{W}_{1} \otimes K_{B}^{-1}\right)$. The $\mathbf{1 6}$ s and $\overline{\mathbf{1 6}}$ s of the $\mathrm{SO}(10)$ on $c$ and $c^{\prime}$ break into the $\mathbf{1 0}$ and $\mathbf{5}$ (and conjugates) of SU(5). Similarly the $\mathbf{1 0}$ over $c_{\Lambda^{2} V_{4}}$ will break into $\mathbf{5}$ and $\overline{\mathbf{5}}$.

- $S_{5}=S_{2} S_{3}=\left(a_{2} X+a_{0} Z^{2}\right)\left(b_{3} Y+b_{2} X Z+b_{0} Z^{3}\right)$ 
Start with the defining short exact sequence,

$$
0 \rightarrow \mathcal{L}_{2} \rightarrow \mathcal{L}_{5} \longrightarrow \mathcal{L}_{3} \longrightarrow 0 \text {. }
$$

The extension morphism of this sequence lives in the following group,

$$
\begin{aligned}
\operatorname{RHom}_{D(X)}\left(\mathcal{L}_{3}, \mathcal{L}_{2}\right) & =\operatorname{RHom}_{D\left(S_{3}\right)}\left(\mathcal{L}_{3},\left.\mathcal{L}_{2}\right|_{S_{2} \cdot S_{3}} \otimes \mathcal{O}\left(S_{3}\right)[-1]\right) \\
& \Downarrow \\
\operatorname{Ext}_{X}^{1}\left(\mathcal{L}_{3}, \mathcal{L}_{2}\right) & =H^{0}\left(S_{2} \cdot S_{3}, \mathcal{L}_{3}^{*} \otimes \mathcal{L}_{2} \otimes \mathcal{O}\left(S_{3}\right)\right) .
\end{aligned}
$$

Since $S_{5}$ intersects with zero section transversely, $\pi_{*} V=0 . H^{1}(V)$ can be computed easily as,

$$
\begin{gathered}
H^{1}(V)=H^{0}\left(R^{1} \pi_{*} V\right), \\
\left.\left.0 \longrightarrow \mathcal{L}_{2}\right|_{c_{2}} \longrightarrow R^{1} \pi_{*} V \longrightarrow \mathcal{L}_{3}\right|_{c_{3}} \longrightarrow 0,
\end{gathered}
$$

where $c_{2}$ and $c_{3}$ are the curves defined by $a_{2}=0$ and $b_{3}=0$. The extension of the sequence above is induced by the extension of the original sequence. The next part as usual is to compute the $R \pi_{*} \Lambda^{2} V$ via antisymmetrized Pontrjagin product

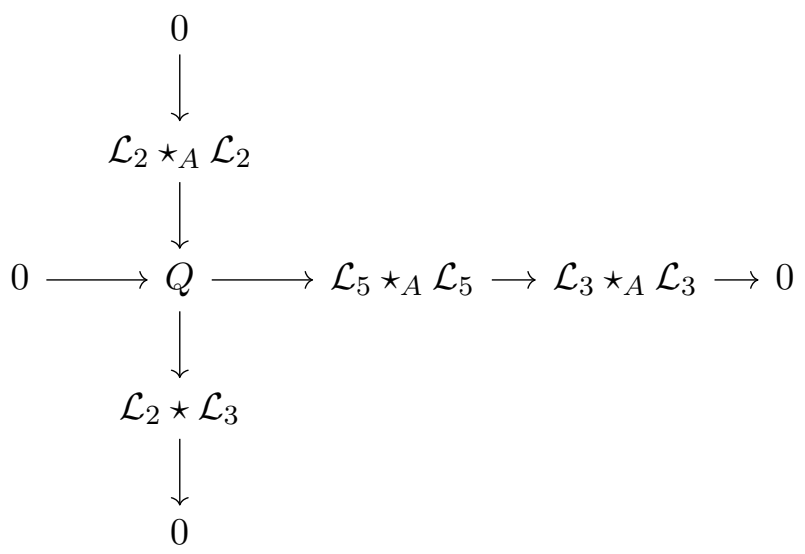

We need to restrict this to the zero section as usual. Remember $\mathcal{L}_{2} \star_{A} \mathcal{L}_{2}=i_{\sigma *} \operatorname{Det}\left(\pi_{*} \mathcal{L}_{2}\right)$, and $L i_{\sigma}^{*} \mathcal{L}_{3} \star_{A} \mathcal{L}_{3}=i_{c_{3} *} \operatorname{Det}\left(\mathcal{L}_{3} \mid \tilde{c}\right) .{ }^{15}$ So we only need to find the restriction of $\mathcal{L}_{2} \star \mathcal{L}_{3}$ on the zero section. Then suppose $S_{2}$ intersect a generic fiber at two points $P_{1}$ and $P_{2}$ $\left(P_{1} \boxplus P_{2}=0\right)$, and $S_{3}$ intersect at $Q_{1}, Q_{2}$ and $Q_{3}$. Then $\mathcal{L}_{2} \star \mathcal{L}_{3}$ is supported on the following points

$$
\begin{array}{lll}
P_{1} \boxplus Q_{1}, & P_{1} \boxplus Q_{2}, & P_{1} \boxplus Q_{3} \\
P_{2} \boxplus Q_{1}, & P_{2} \boxplus Q_{2}, & P_{2} \boxplus Q_{3} .
\end{array}
$$

So the zero section intersect when one of these points say $P_{1} \boxplus Q_{1}$ becomes zero, which means $P_{2}=\mathcal{Q}_{1}$. We conclude that the curve $\tau^{*} S_{2} \cdot S_{3}$ maps to the "matter curve" of $\left.\mathcal{L}\right) 2 \star \mathcal{L}_{3}$

\footnotetext{
${ }^{15} \tilde{c}$ is the double cover of $c_{3}$ define in 3.1.1.
} 
(which is a $2: 1$ map as will be mentioned shortly). Therefore the derived pushforwards of $\Lambda^{2} V$ is given by

$$
R \pi_{*} \Lambda^{2} V=\operatorname{Det}\left(\pi_{*} \mathcal{L}_{2}\right) \otimes K_{B}^{-2} \oplus \mathcal{H} \otimes K_{B}^{-1}[-1]
$$

where $\mathcal{H}$ is

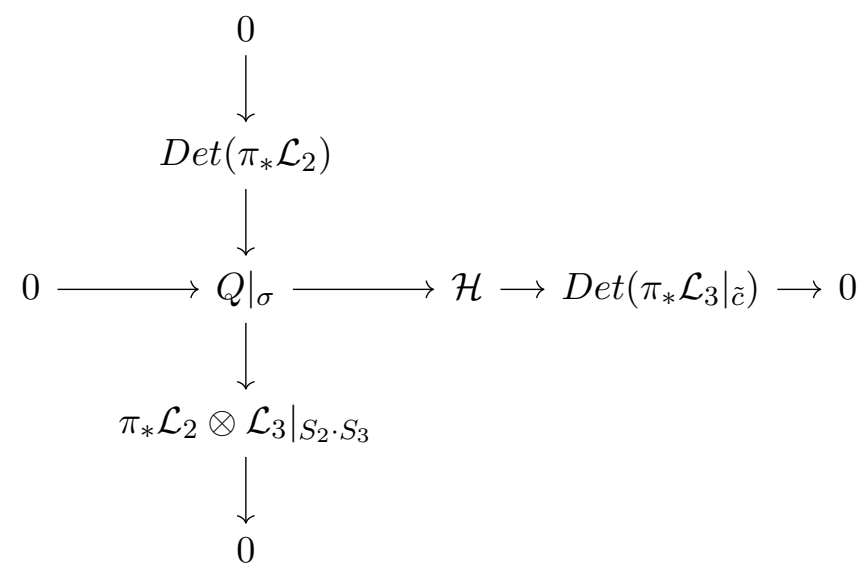

where $\tilde{c}$ is the double cover of $b_{3}=0$ defined previously for $E_{6}$ models, and $S_{2} \cdot S_{3}$ is a double cover of ${ }^{16}$

$$
c_{\Lambda^{2} V_{5}}:=a_{0}^{3} b_{3}^{2}+a_{0}^{2} a_{2} b_{2}^{2}-2 a_{0} a_{2}^{2} b_{0} b_{2}+a_{2}^{3} b_{0}^{2}+a 2^{2} b_{3}^{2}\left(a_{0} F-a_{2} G\right) .
$$

This model has point like $\mathbf{1 0 - \overline { \mathbf { 5 } }} \overline{\mathbf{5}}$ candidates on the intersection locus of the matter curves.

$$
H^{0}\left(c_{2},\left.\mathcal{L}_{2}\right|_{c_{2}}\right) \otimes H^{0}\left(c_{3},\left.\mathcal{L}_{3} \star_{A} \mathcal{L}_{3}\right|_{c_{3}}\right) \otimes H^{0}\left(\Lambda^{2} V_{5},\left.\mathcal{L}_{2} \star \mathcal{L}_{3}\right|_{c_{\Lambda^{2} V_{5}}}\right) \longrightarrow \mathbb{C}
$$

The intuition is similar to the $\mathrm{SU}(4)$ bundle with reducible spectral cover mentioned before. However, one should be careful whether these cohomologies contribute in $H^{1}\left(\Lambda^{2} V\right)$. In particular, the extension of the sequence $Q$ can be non-zero.

There are also pointlike $\mathbf{1 0 - 1 0 - 5}$ in this case

$$
H^{0}\left(\left.\mathcal{L}_{2}\right|_{c_{2}}\right) \otimes H^{0}\left(\left.\mathcal{L}_{3}\right|_{c_{3}}\right) \otimes H^{0}(\mathcal{H}) \rightarrow \mathbb{C}
$$

where the first two factors are $H^{1}(V)$ subgroups (possibly zero) and the third one is the $H^{1}\left(\Lambda^{3} V\right)$ contribution. We have used the relation $\Lambda^{2} V^{*} \simeq \Lambda^{3} V$ and the fact that $\Phi\left(V^{*}\right)=$ $\tau^{*} \Phi(V)$ to compute the relevant part of $H^{1}\left(\Lambda^{3} V\right)$.

The F-theory analysis is very similar to $S_{4}=S_{2} S_{2}^{\prime}$ case in 3.2.2. The F-theory CalabiYau has a $\mathrm{SU}(6)$ singularity corresponding to the $S(\mathrm{U}(2) \times \mathrm{U}(3))$ on the heterotic (the extra $\mathrm{U}(1)$ is anomalous) when the extension in the defining short exact sequence was zero. Before turning on the extension, there are $\mathbf{2 0}$ hypermultiplets of SU(6) in $a_{2}=0, \mathbf{1 5}$ and $\overline{\mathbf{1 5}}$ in $b_{3}=0$, and $\mathbf{6}$ and $\overline{\mathbf{6}}$ in $c_{\lambda^{2} V_{5}} .{ }^{17}$ So at the intersections, there are 20-15-6 and 20-15$\overline{6}$ Yukawa couplings. Turning on the Higgs field, corresponds to turning on the extension

\footnotetext{
${ }^{16} \mathrm{~F}$ and $G$ are the defining polynomials in the Weierstrass model.

${ }^{17}$ In heterotic picture, the $\mathbf{6}$ and $\overline{\mathbf{6}}$ hypermultiplets live in either the deformations of the vector bundle (in particular the extension morphisms) and in $H^{1}\left(\Lambda^{2} V\right)$.
} 

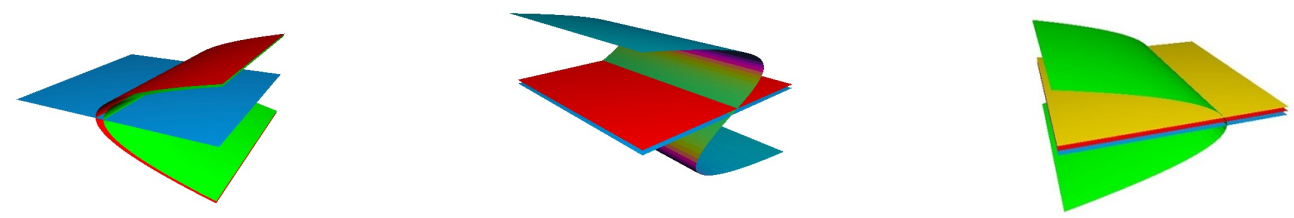

Figure 12. Degree five spectral covers with non-reduced components.

of the defining short exact sequence, and it breaks $\mathrm{SU}(6)$ to $\mathrm{U}(1)$. The Yukawa couplings at the intersections decompose into the $\mathbf{1 0 - 1 0 - 5}$ and $\mathbf{1 0 - 5 - 5}$ couplings, in agreement with our heterotic calculations. ${ }^{18}$ We ignore the details at this point.

\subsubsection{Spectral cover with non-reduced components}

In this final subsection we study the degree five spectral cover with non-reduced components, figure 12. These cases correspond to the following spectral sheaves

$$
\begin{array}{lll}
S_{5}=Z\left(a_{2} X+a_{0} Z^{2}\right)^{2}, & \left.0 \longrightarrow \mathcal{W}_{1} \longrightarrow \mathcal{L}_{5} \longrightarrow \mathcal{L}_{4}\right|_{\left(S_{2}\right)^{2}} \longrightarrow 0, \\
S_{5}=Z^{2}\left(a_{3} Y+a_{2} X Z+a_{0} Z^{3}\right), & \left.0 \longrightarrow \mathcal{W}_{1} \longrightarrow \mathcal{L}_{5} \longrightarrow \mathcal{L}_{4}\right|_{Z S_{3}} \longrightarrow 0, \\
S_{5}=Z^{3}\left(a_{2} X+a_{0} Z^{2}\right) & \left.0 \longrightarrow \mathcal{W}_{1} \longrightarrow \mathcal{L}_{5} \longrightarrow \mathcal{L}_{4}\right|_{Z^{2} S_{2}} \longrightarrow 0 .
\end{array}
$$

The F-theory picture of these $\mathrm{SU}(5)$ heterotic models, roughly speaking, can be explained by adding an extra $\mathrm{U}(1)$ flux to the corresponding " $\mathrm{U}(4)$ " model inside the 7-brane and consider its interaction with the bulk/localized zero modes that are already there. Since we have briefly done a similar calculation of this type the previous sections, we ignore the F-theory dual in this subsection.

- $S_{5}=Z\left(a_{2} X+a_{0} Z^{2}\right)^{2}$

This case corresponds to adding a zero section to the spectral cover of (3.72). So the parameters of the defining sequence is (modulo the defining extensions of $\mathcal{L}_{4}$ )

$$
\begin{aligned}
\operatorname{RHom}_{X}\left(\mathcal{L}_{4}, \mathcal{W}_{1}\right) & =\operatorname{RHom}_{c}\left(\left.\mathcal{L}_{4}\right|_{c}, \mathcal{W}_{1} \otimes \mathcal{O}_{c}(c)[-1]\right) \\
& \Downarrow \\
\operatorname{Ext}_{X}^{1}\left(\mathcal{L}_{4}, \mathcal{W}_{1}\right) & =\operatorname{Hom}_{c}\left(\left.\mathcal{L}_{4}\right|_{c}, \mathcal{W}_{1} \otimes \mathcal{O}_{c}(c)\right) .
\end{aligned}
$$

Next, to compute $H^{1}(V)$ the derived pushforwards $R \pi_{*} V$ can be used

$$
\begin{aligned}
0 \longrightarrow & \mathcal{W}_{1} \otimes K_{B}^{-1} \stackrel{\simeq}{\longrightarrow} \pi_{*} V \longrightarrow 0 \\
& \left.\longrightarrow \mathcal{W}_{1} \longrightarrow R^{1} \pi_{*} V \longrightarrow \mathcal{L}_{4}\right|_{c} \longrightarrow 0 .
\end{aligned}
$$

\footnotetext{
${ }^{18}$ As mentioned before one should check whether the hypermultiplets in the Yukawa couplings remain massless after Higgsing or not, this is exactly what we mean by checking whether the zero modes are killed by the extension morphisms.
} 
The cohomologies of $\Lambda^{2} V$ can be computed by using the derived pushforward of it. For that, the antisymmetrized Pontrjagin product of $\mathcal{L}_{5}$ has to be computed

$$
0 \longrightarrow \pi^{*} \pi_{*} \mathcal{W}_{1} \otimes \mathcal{L}_{4} \rightarrow \mathcal{L}_{5} \star_{A} \mathcal{L}_{5} \rightarrow \mathcal{L}_{4} \star_{A} \mathcal{L}_{4} \rightarrow 0
$$

As a by product that can help at some point, it is easy to read the spectral cover of $\Lambda^{2} V$

$$
S_{\Lambda^{2} V}=Z^{4}\left(a_{2} X+a_{0} Z^{2}\right)^{2}\left(4 C_{t o r} a_{2} X+a_{0} Z^{2}\right)
$$

where $c_{\text {tor }}$ is defined above (3.77). We've proved $R \pi_{*} \Lambda^{2} V=L i_{\sigma}^{*} \mathcal{L}_{5} \star_{A} \mathcal{L}_{5} \otimes K_{B}^{-1}$,

$$
\begin{gathered}
0 \longrightarrow L^{-1} i_{\sigma}^{*} \mathcal{L}_{5} \star_{A} \mathcal{L}_{5} \longrightarrow L^{-1} i_{\sigma}^{*} \mathcal{L}_{4} \star_{A} \mathcal{L}_{4} \\
\left.\hookrightarrow\left(\mathcal{W}_{1} \otimes \mathcal{L}_{4}\right)\right|_{c} \longrightarrow i_{\sigma}^{*} \mathcal{L}_{5} \star_{A} \mathcal{L}_{5} \longrightarrow i_{\sigma}^{*} \mathcal{L}_{4} \star_{A} \mathcal{L}_{4} \longrightarrow 0
\end{gathered}
$$

The only relevant terms for the Yukawa coupling 10-10-5 is the second term, which gives a localized Yukawa coupling on $c$ is

$$
H^{1}\left(\mathcal{W}_{1} \otimes K_{B}^{-1}\right) \otimes H^{0}\left(\left.\mathcal{L}_{4}\right|_{c}\right) \longrightarrow H^{1}\left(\left.\left(\mathcal{W}_{1} \otimes \mathcal{L}_{4}\right)\right|_{c} \otimes K_{B}^{-1}\right)
$$

There are also possible $\overline{\mathbf{5}}-\overline{\mathbf{5}} \mathbf{- 1 0}$ non localized Yukawa couplings. First notice that

$$
c_{1}\left(\pi_{*} \mathcal{L}_{n}\right)=5 K_{B}
$$

So,

$$
\mathcal{W}_{1} \otimes \operatorname{Det}\left(\pi_{*} \mathcal{L}_{2}\right) \otimes \operatorname{Det}\left(\pi_{*} \mathcal{L}_{2}^{\prime}\right)=K_{B}^{5}
$$

Therefore the are following morphisms,

$$
H^{1}\left(\mathcal{W}_{1} \otimes K_{B}^{-1}\right) \otimes H^{1}\left(\operatorname{Det}_{*} \mathcal{L}_{2} \otimes K_{B}^{-2}\right) \otimes H^{0}\left(\operatorname{Det}_{*} \mathcal{L}_{2}^{\prime} \otimes K_{B}^{-1}\right) \longrightarrow \mathbb{C},
$$

or,

$$
H^{1}\left(\mathcal{W}_{1} \otimes K_{B}^{-1}\right) \otimes H^{0}\left(\operatorname{Det}_{*} \mathcal{L}_{2} \otimes K_{B}^{-1}\right) \otimes H^{1}\left(\operatorname{Det}_{*} \mathcal{L}_{2}^{\prime} \otimes K_{B}^{-2}\right) \longrightarrow \mathbb{C},
$$

or, ${ }^{19}$

$$
H^{0}\left(\mathcal{W}_{1}\right) \otimes H^{1}\left(\operatorname{Det}_{*} \mathcal{L}_{2} \otimes K_{B}^{-2}\right) \otimes H^{1}\left(\operatorname{Det}_{*} \mathcal{L}_{2}^{\prime} \otimes K_{B}^{-2}\right) \longrightarrow \mathbb{C}
$$

In these cases we should check whether $H^{i}\left(\operatorname{Det}_{*} \mathcal{L}_{2} \otimes K_{B}^{-1-i}\right)$ and $H^{i}\left(\operatorname{Det}_{*} \mathcal{L}_{2}^{\prime} \otimes K_{B}^{-1-i}\right)$ contribute in $H^{1}\left(\Lambda^{2} V\right)$. From the analysis of the degree four spectral cover it seems the coboundary morphisms don't kill the elements in the corresponding cohomologies and therefore all of the Yukawa couplings above are non-vanishing. A detailed example of this type will be given later in this paper.

\footnotetext{
${ }^{19}$ The third possibility must be zero for when $K_{B}^{-1}$ is effective. This because when $H^{0}\left(\mathcal{W}_{1}\right)$ is non-zero the $\left.H^{(} \mathcal{W}_{1} \otimes K_{B}^{-1}\right)$ can be non-zero too, which means $H^{0}(V)$ is non-zero, then $V$ is unstable.
} 
- $S_{5}=Z^{2}\left(a_{3} Y+a_{2} X Z+a_{0} Z^{3}\right)$

This is the same as adding a zero section to the spectral cover $S_{4}$ in (3.42). The extension morphism of the spectral sheaf (modulo the parts that correspond to the deformations of $\mathcal{L}_{4}$ ) live the following group

$$
\operatorname{RHom}_{X}\left(\mathcal{L}_{4}, \mathcal{W}_{1}\right)=\operatorname{RHom}_{B}\left(\mathcal{F} \oplus \mathcal{L}_{1} \otimes K_{B}^{-1}[+1], \mathcal{W}_{1}\right),
$$

which leads to the following long exact sequence

$$
0 \rightarrow \operatorname{Ext}_{B}^{1}\left(\mathcal{F}, \mathcal{W}_{1}\right) \rightarrow \operatorname{Ext}_{X}^{1}\left(\mathcal{L}_{4}, \mathcal{W}_{1}\right) \rightarrow \operatorname{Ext}^{0}\left(\mathcal{L}_{1} \otimes K_{B}^{-1}, \mathcal{W}_{1}\right) \rightarrow \operatorname{Ext}_{B}^{2}\left(\mathcal{F}, \mathcal{W}_{1}\right)
$$

where $\mathcal{F}$ is defined before,

$$
0 \longrightarrow \mathcal{L}_{1} \longrightarrow \mathcal{F} \longrightarrow i_{c *} \mathcal{L}_{3} \longrightarrow 0
$$

The cohomology of $V$ can be computed by the derived pushforward $R \pi_{*} V=L i_{\sigma}^{*} \mathcal{L}_{5}[-1]$,

$$
\begin{aligned}
& 0 \longrightarrow \mathcal{W}_{1} \otimes K_{B}^{-1} \longrightarrow L^{-1} i_{\sigma}^{*} \mathcal{L}_{5} \longrightarrow \mathcal{L}_{1} \otimes K_{B}^{-1} \\
& \longrightarrow \mathcal{W}_{1} \longrightarrow L^{0} i_{\sigma}^{*} \mathcal{L}_{5} \longrightarrow \mathcal{F} \longrightarrow 0
\end{aligned}
$$

Therefore,

$$
\begin{array}{r}
L i_{\sigma}^{*} \mathcal{L}_{5}=\overline{\mathcal{N}} \oplus \mathcal{W}_{1} \otimes K_{B}^{-1}[+1] . \\
\left.0 \longrightarrow \mathcal{W}_{1}\right|_{\{\mathcal{E} x t=0\}} \rightarrow \overline{\mathcal{N}} \rightarrow \mathcal{F} \longrightarrow 0 .
\end{array}
$$

As always the next step is computing $R \pi_{*} \Lambda^{2} V=L i_{\sigma}^{*} \mathcal{L}_{5} \star_{A} \mathcal{L}_{5} \otimes K_{B}^{-1}[-1]$. So let us compute $\mathcal{L}_{5} \star_{A} \mathcal{L}_{5}$

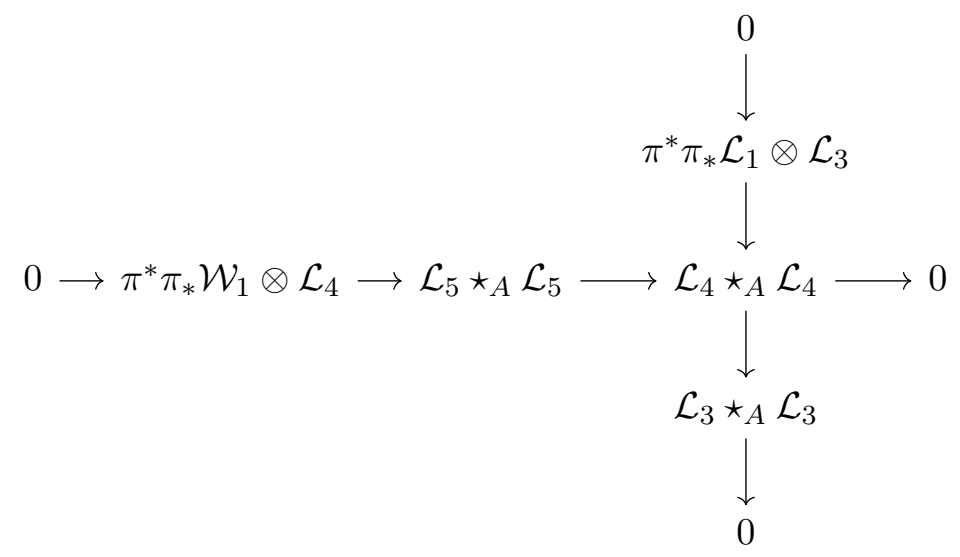

One can read the spectral cover of the associated bundle easily

$$
S_{\Lambda^{2} V}=Z\left(a_{3} Y+a_{2} X Z+a_{0} Z^{3}\right)^{2}\left(-a_{3} Y+a_{2} X Z+a_{0} Z^{3}\right) .
$$


Anyways, the restriction over the zero section is given by the following formula

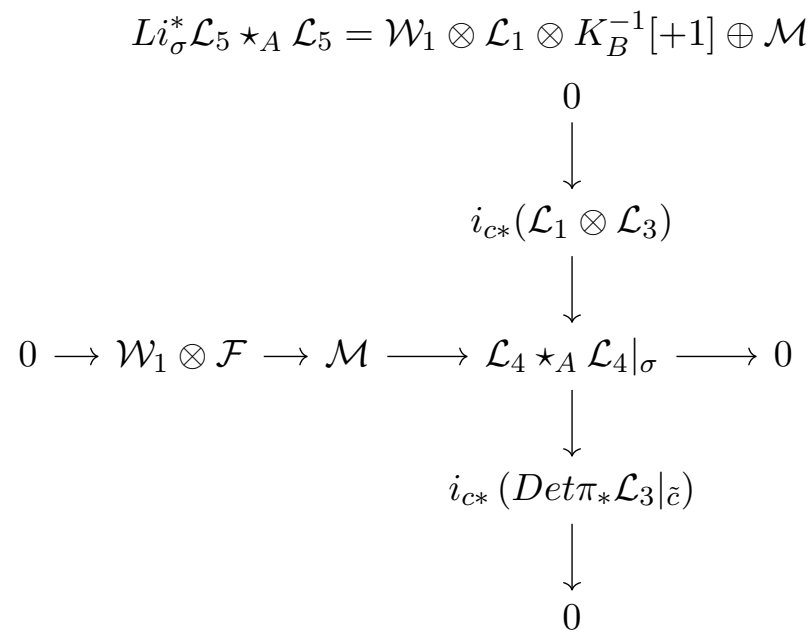

There is a "candidate" for Yukawa coupling 10-10-5

$$
H^{1}\left(\mathcal{W}_{1} \otimes K_{B}^{-1}\right) \otimes H^{0}(\mathcal{F}) \longrightarrow H^{1}\left(\mathcal{W}_{1} \otimes \mathcal{F} \otimes K_{B}^{-1}\right)
$$

The coboundary map $H^{0}\left(i_{c *}\left(\mathcal{L}_{1} \otimes \mathcal{L}_{3}\right) \otimes K_{B}^{-1}\right) \longrightarrow H^{1}\left(\mathcal{W}_{1} \otimes \mathcal{F} \otimes K_{B}^{-1}\right)$ is induced by the extension of $\mathcal{W}_{1}$ and $\mathcal{L}_{3}$ in the original sequence. So it is non-zero generically, and to see whether 10-10-5 localized, one needs to check whether the corresponding terms are not killed by this coboundary map.

For $\overline{\mathbf{5}} \overline{\mathbf{5}}-\mathbf{1 0}$ There are also candidates on $c$. For this case we should use the relation between the line bundles $\mathcal{W}_{1}, \mathcal{L}_{1}, \operatorname{Det}\left(\pi_{*} \mathcal{L}_{3}\right)$. The relation is,

$$
\left.\left.\left.\mathcal{W}_{1}\right|_{c} \otimes \mathcal{L}_{1}\right|_{c} \otimes \mathcal{L}_{3}\right|_{c} \otimes \operatorname{Det}\left(\left.\pi_{*} \mathcal{L}_{3}\right|_{\tilde{c}}\right)=K_{c} \otimes K_{B}^{+3},
$$

where $K_{c}$ is the canonical bundle of the curve $c$. This relation can be derived as follows. First start with the, by now, well known relation,

$$
c_{1}\left(\pi_{*} \mathcal{L}_{5}\right)=5 c_{1}\left(K_{B}\right)=c_{1}\left(\mathcal{W}_{1}\right)+c_{1}\left(\mathcal{L}_{1}\right)+c_{1}\left(\pi_{*} \mathcal{L}_{3}\right) .
$$

Note that the restriction of $S_{3}$ over $p i^{-1} c$ is reducible, therefore $i_{\pi^{-1} c^{*}}^{*} \mathcal{L}_{3}$ can be represented by a short exact sequence,

$$
\left.\left.0 \longrightarrow \mathcal{L}_{3}\right|_{c} \otimes \mathcal{O}_{c}(-\tilde{c} \cdot c) \longrightarrow i_{\pi^{-1}}^{*} \mathcal{L}_{3} \longrightarrow \mathcal{L}_{3}\right|_{\tilde{c}} \longrightarrow 0 .
$$

To compute $\mathcal{O}_{c}(-\tilde{c} \cdot c)$, note that the intersection locus of $c$ and $\tilde{c}$ is in one-to-one correspondence to the intersection locus of $a_{3}=a_{2}=0$ in the base. So one gets

$$
\mathcal{O}_{c}(-\tilde{c} \cdot c)=K_{c}^{-1} \otimes K_{B}^{2},
$$

where we used the adjunction formula for the curve $c$. Consequently we get the a simle relation for $c_{1}\left(\pi_{*} \mathcal{L}_{3}\right)$ when it is restricted over $\pi^{-1} c$,

$$
i_{\pi^{-1} c}^{*} c_{1}\left(\pi_{*} \mathcal{L}_{3}\right)=c_{1}\left(i_{\pi^{-1} c}^{*} \pi_{*} \mathcal{L}_{3}\right)=2 c_{1}\left(K_{B}\right)-K_{c}+c_{1}\left(\left.\mathcal{L}_{3}\right|_{c}\right)+c_{1}\left(\pi_{*} \mathcal{L}_{3} \mid \tilde{c}\right) .
$$


Putting everything together one gets the advertised relation. Then it is easy to see that there are actually possible $\overline{\mathbf{5}} \overline{\mathbf{5}} \mathbf{- 1 0}$ from the interaction of the bulk and localized zero modes,

$$
\begin{aligned}
& H^{0}\left(\left.\mathcal{L}_{c}\right|_{c}\right) \otimes H^{1}\left(\mathcal{W}_{1} \otimes \mathcal{L}_{1} \otimes K_{B}^{-2}\right) \otimes H^{0}\left(\operatorname{Det}\left(\pi_{*} \mathcal{L}_{3} \mid \tilde{c}\right)\right) \longrightarrow H^{1}\left(K_{c}\right) \simeq H^{0}\left(\mathcal{O}_{c}\right)=\mathbb{C} . \\
& \text { - } S_{5}=Z^{3}\left(a_{2} X+a_{0} Z^{2}\right)
\end{aligned}
$$

This corresponds to adding a zero section to the degree four spectral cover of (3.62). Start with the extension group as usual

$$
\begin{aligned}
\operatorname{RHom}_{D(X)}\left(\mathcal{L}_{4}, \mathcal{W}_{1}\right) & =\operatorname{RHom}_{D(B)}\left(\operatorname{Li}_{\sigma}^{*} \mathcal{L}_{4}, \mathcal{W}_{1}\right) \\
& =\operatorname{RHom}_{D(B)}\left(\mathcal{U}_{1} \otimes K_{B}^{-1}[+1] \oplus \overline{\mathcal{F}}, \mathcal{W}_{1}\right)
\end{aligned}
$$

This leads to a long exact sequence

$$
0 \longrightarrow \operatorname{Ext}_{B}^{1}\left(\overline{\mathcal{F}}, \mathcal{W}_{1}\right) \longrightarrow \operatorname{Ext}_{D(X)}^{1}\left(\mathcal{L}_{4}, \mathcal{W}_{1}\right) \longrightarrow \operatorname{Ext}_{B}^{0}\left(\mathcal{U}_{1} \otimes K_{B}^{-1}, \mathcal{W}_{1}\right) \rightarrow \operatorname{Ext}_{B}^{2}\left(\overline{\mathcal{F}}, \mathcal{W}_{1}\right)
$$

Again the first term on the left contains elements that correspond to gluing the line bundles to a rank two vector bundle on the zero section. We keep these terms off. It also contains the gluing between $\mathcal{W}_{1}$ and the line bundle over $S_{2}$. The third term corresponds to a sheaf with numeric rank 1 on the non-reduced surface $Z^{2}=0$. This term is generically on to construct generic spectral sheaves. Next let us compute $R \pi_{*} V=L i_{\sigma}^{*} \mathcal{L}_{5}[-1]$

$$
\begin{aligned}
0 \longrightarrow & \mathcal{W}_{1} \otimes K_{B}^{-1} \longrightarrow L^{-1} i_{\sigma}^{*} \mathcal{L}_{5} \longrightarrow \mathcal{U}_{1} \otimes K_{B}^{-1} \\
& \longrightarrow \mathcal{W}_{1} \longrightarrow L^{0} i_{\sigma}^{*} \mathcal{L}_{5} \longrightarrow \overline{\mathcal{F}} \longrightarrow 0 .
\end{aligned}
$$

Therefore,

$$
L i_{\sigma}^{*} \mathcal{L}_{5}=\mathcal{W}_{1} \otimes K_{B}^{-1}[+1] \oplus \mathcal{J},\left.0 \longrightarrow \mathcal{W}_{1}\right|_{\mathcal{E}_{x t_{5}=0}} \rightarrow \mathcal{J} \longrightarrow \overline{\mathcal{F}} \longrightarrow 0 .
$$

The next part is computing the $\mathcal{L}_{5} \star_{A} \mathcal{L}_{5}$

$$
0 \longrightarrow \pi^{*} \pi_{*} \mathcal{W}_{1} \otimes \mathcal{L}_{4} \rightarrow \mathcal{L}_{5} \star_{A} \mathcal{L}_{5} \rightarrow \mathcal{L}_{4} \star_{A} \mathcal{L}_{4} \rightarrow 0 .
$$

We can also read the spectral cover of $\Lambda^{2} V$

$$
S_{\Lambda^{2} V}=Z^{4}\left(a_{2} X+a_{0} Z^{2}\right)^{3} .
$$

Restrict to the zero section to get $R \pi_{*} \Lambda^{2} V=L i_{\sigma}^{*} \mathcal{L}_{5} \star_{A} \mathcal{L}_{5} \otimes K_{B}^{-1}[-1]$

$$
\begin{aligned}
0 \rightarrow \mathcal{W}_{1} \otimes \mathcal{U}_{1} \otimes K_{B}^{-1} \longrightarrow L^{-1} i_{\sigma}^{*} \mathcal{L}_{5} \star_{A} \mathcal{L}_{5} \longrightarrow L^{-1} i_{\sigma}^{*} \mathcal{L}_{4} \star_{A} \mathcal{L}_{4} \\
\\
\longrightarrow \mathcal{W}_{1} \otimes \overline{\mathcal{F}} \longrightarrow L^{0} i_{\sigma}^{*} \mathcal{L}_{5} \star_{A} \mathcal{L}_{5} \longrightarrow L^{0} i_{\sigma}^{*} \mathcal{L}_{4} \star_{A} \mathcal{L}_{4} \longrightarrow 0
\end{aligned}
$$

The coboundary map can receive non-zero contributions from $\operatorname{Hom}_{D(B)}\left(\mathcal{U}_{1} \otimes K_{B}^{-1}, \mathcal{W}_{1}\right)$. Therefore if the following terms

$$
H^{1}\left(\mathcal{W}_{1} \otimes K_{B}^{-1}\right) \otimes H^{0}(\overline{\mathcal{F}}) \longrightarrow H^{1}\left(\mathcal{W}_{1} \otimes \overline{\mathcal{F}} \otimes K_{B}^{-1}\right)
$$


have non-zero contribution in $H^{1}\left(L^{0} i_{\sigma}^{*} \mathcal{L}_{5} \star_{A} \mathcal{L}_{5}\right)$, then we can have Yukawa coupling 1010-5. Note that theses couplings are not coming from localized modes.

There are three possibilities for the $\overline{\mathbf{5}} \overline{\mathbf{5}} \mathbf{- 1 0}$ coupling due to the following relation,

$$
\mathcal{W}_{1} \otimes \mathcal{U}_{1} \otimes \mathcal{L}_{1} \otimes \operatorname{Det}\left(\pi_{*} \mathcal{L}_{2}\right)=K_{B}^{5}
$$

The cohomologies are,

$$
\begin{aligned}
& H^{1}\left(\mathcal{W}_{1} \otimes \mathcal{U}_{1} \otimes K_{B}^{-2}\right) \otimes H^{1}\left(\operatorname{Det}_{*} \mathcal{L}_{2} \otimes K_{B}^{-2}\right) \otimes H^{0}\left(\mathcal{L}_{1}\right) \longrightarrow \mathbb{C}, \\
& H^{1}\left(\mathcal{L}_{1} \otimes \mathcal{U}_{1} \otimes K_{B}^{-2}\right) \otimes H^{0}\left(\operatorname{Det}_{*} \mathcal{L}_{2} \otimes K_{B}^{-1}\right) \otimes H^{1}\left(\mathcal{W}_{1} \otimes K_{B}^{-1}\right) \longrightarrow \mathbb{C}, \\
& H^{0}\left(\mathcal{L}_{1} \otimes \mathcal{U}_{1} \otimes K_{B}^{-1}\right) \otimes H^{1}\left(\operatorname{Det}_{*} \mathcal{L}_{2} \otimes K_{B}^{-2}\right) \otimes H^{1}\left(\mathcal{W}_{1} \otimes K_{B}^{-1}\right) \longrightarrow \mathbb{C},
\end{aligned}
$$

Of course one needs to check whether the elements of these cohomologies contribute in $H^{1}\left(\Lambda^{2} V\right) \otimes H^{1}\left(\Lambda^{2} V\right) \otimes H^{1}(V)$.

\section{Vertical component}

The cases with vertical components are somewhat subtle. This is because of the existence of singular fibers. In case the ambiguity is not too harsh, as in the completely degenerate spectral cover, we can still use the spectral data. However, it is more satisfying if we can give an alternative way to compute the cohomologies of $\Lambda^{2} V$ that works in any case that spectral cover contains vertical components. As in the previous cases, we first consider a degree two spectral cover as a warm up example, and then study the effect of these vertical components for the $\mathrm{SU}(3), \mathrm{SU}(4)$ and $\mathrm{SU}(5)$ examples in detail.

Consider a spectral cover of the form $\alpha\left(b_{2} X+b_{0} Z^{2}\right)$ which is double cover with a vertical component,

$$
S_{2}=\alpha\left(b_{2} X+b_{0} Z^{2}\right),
$$

where $\alpha, b_{0}$ and $b_{2}$ are suitable polynomials in the base. $b_{0}$ and $b_{2}$ should be generic without common factors, and $\alpha$ can be reducible but shouldn't have factors with multiplicities more than one. First assume that $\alpha$ is a generic irreducible polynomial. Then the spectral cover is now a union of a smooth vertical component and a smooth double cover. The spectral sheaf, without loss of generality can be written as,

$$
0 \rightarrow \mathcal{L} 1_{V} \rightarrow \mathcal{L}_{2} \longrightarrow \mathcal{L}_{2}^{\prime} \longrightarrow 0
$$

where $\mathcal{L}_{2}$ is the spectral sheaf as usual. ${ }^{20} \quad \mathcal{L} 1_{V}$ is a rank one coherent sheaf over the vertical components $\alpha=0$ (call it $S_{V}$ ), and $\mathcal{L}_{2}^{\prime}$ is rank one coherent sheaf supported over $b_{2} X+b_{0} Z^{2}=0$. Since the inverse Fourier transform of the spectral sheaf must be a vector bundle, the coherent sheaves $\mathcal{L} 1_{V}$ and $\mathcal{L}_{2}^{\prime}$ can have singularity only at the intersection locus of the components, however as we will argue later, these singularities do not contribute in the Yukawa couplings, therefore from now on we will assume that the restriction of the

\footnotetext{
${ }^{20}$ We don't write the inclusion pushforward explicitly from now on, unless it causes confusion. For example instead of $i_{S_{2} *} \mathcal{L}_{2}$ we simply write $\mathcal{L}_{2}$.
} 
spectral sheaf over each component is smooth. Therefore $\mathcal{L} 1_{V}$ and $\mathcal{L}_{2}^{\prime}$ are line bundles. The extension group of this sequence is also important to know,

$$
\operatorname{Ext}^{1}\left(\mathcal{L}_{2}^{\prime}, \mathcal{L} 1_{V}\right)=H^{0}\left(S_{2}^{\prime} \cap S_{V}, \mathcal{L} 1_{V} \otimes \mathcal{L}_{2}^{*} \otimes \mathcal{O}\left(+S_{2}^{\prime}\right)\right) .
$$

The pushforwards of $V$ can be computed easily as before,

$$
\begin{gathered}
R \pi_{*} V=i_{\sigma}^{*} \mathcal{L}_{2}, \\
\left.\left.0 \longrightarrow \mathcal{L} 1_{V}\right|_{\sigma} \longrightarrow i_{\sigma}^{*} \mathcal{L}_{2} \longrightarrow \mathcal{L}_{2}^{\prime}\right|_{\sigma \cap S_{2}^{\prime}} \longrightarrow 0
\end{gathered}
$$

where the first sheaf is supported on the curve $\alpha=0$ (call it $c_{1}^{m}$ ) and the third sheaf is supported on $b_{2}=0$ (call it $\left.c_{2}^{m}=0\right)$.

Before continuing to the Pontrjagin product some comments about this spectral sheaf should be added. The first comments is that as usual the vertical components show up in the spectral data when the corresponding bundle is unstable on that locus. By similar arguments mentioned in [81] one can show since the vertical component is reduced and $\mathcal{L} 1_{V}$ is a line bundle over this component, the dual vector bundle must become a direct sum of line bundles on $S_{V}$ one with relative degree -1 and the other one with relative degree $+1 .{ }^{21}$ The summand with positive degree do not contribute in the spectral sheaf (due to the flatness of the projection maps and smoothness of the bundle $V$ ). Then a well known Fourier-Mukai property ([82] 6.34) shows that the restriction of $\mathcal{L}_{2}$ on $S_{V}$ must have relative degree +1 . Since $\mathcal{L}_{2}^{\prime}$ is supported over a double cover, the relative degree of $\mathcal{L} 1_{V}$ must be -1 (because 2-1=1). ${ }^{22}$ The second comment is that as before $c_{1}\left(\pi_{*} \mathcal{L}_{2}\right)=2 c_{1}\left(K_{B}\right)$. This should put a constraint on $\mathcal{L}_{2}^{\prime}$ which will be useful later.

$$
0 \longrightarrow \pi_{*} \mathcal{L}_{2} \longrightarrow \pi_{*} \mathcal{L}_{2}^{\prime} \longrightarrow i_{c_{1}^{m}} R^{1} \pi_{*} \mathcal{L} 1_{V} \longrightarrow 0
$$

So one can show,

$$
c_{1}\left(\pi_{*} \mathcal{L}_{2}^{\prime}\right)=2 c_{1}\left(K_{B}\right)+\left[c_{1}^{m}\right] .
$$

The next task is to compute $\mathcal{L}_{2} \star_{A} \mathcal{L}_{2}$. But, as mentioned, dealing with the Pontrjagin product that involves the $\mathcal{L} 1_{V}$ is somewhat ambiguous, and it is better to avoid this. The idea is that we know how to deal with the $\mathcal{L}_{2}^{\prime}$ part. So at this point we do an inverse Fourier transform and try to compute $\Lambda^{2} V$ in terms directly. More clearly, since the degree of $\mathcal{L} 1_{V}$ is $(-1)$ over the fibers, the inverse Fourier transform gives,

$$
0 \rightarrow V \rightarrow \bar{V} \rightarrow i_{c_{1}^{m} *} \mathcal{F} \rightarrow 0
$$

where $\mathcal{F}$ is a line bundle with relative degree $(+1)$ over the fibers. Since we can compute $\mathcal{L}_{2}^{\prime} \star_{A} \mathcal{L}_{2}^{\prime}$ we can compute $\Lambda^{2} \bar{V}$. To use the above sequence to compute $\Lambda^{2} V$ we have to dualize the sequence above,

$$
0 \longrightarrow \bar{V}^{*} \longrightarrow V^{*} \longrightarrow i_{c_{1}^{m} *} \mathcal{F}^{*} \otimes \mathcal{O}\left(c_{1}^{m}\right) \longrightarrow 0 .
$$

\footnotetext{
${ }^{21}$ More clearly $\left.V\right|_{S_{V}}=\mathcal{O}(\sigma+A) \oplus \mathcal{O}(-\sigma+B)$ where $A$ and $B$ are divisors in the base.

${ }^{22}$ This is good because for the specific Fourier-Mukai that we are using here (defined with the Poincare sheaf as the kernel) the pushforward of $\mathcal{L}_{2}$ to the base must correspond to the restriction of $V$ to zero section $\sigma$, and hence it should be smooth. However if the relative degree of $\mathcal{L} 1_{V}$ was positive, the pushforward $\pi_{*} \mathcal{L}_{2}$ would be singular.
} 
Now, as explained in the A, one can derive the following sequence from this,

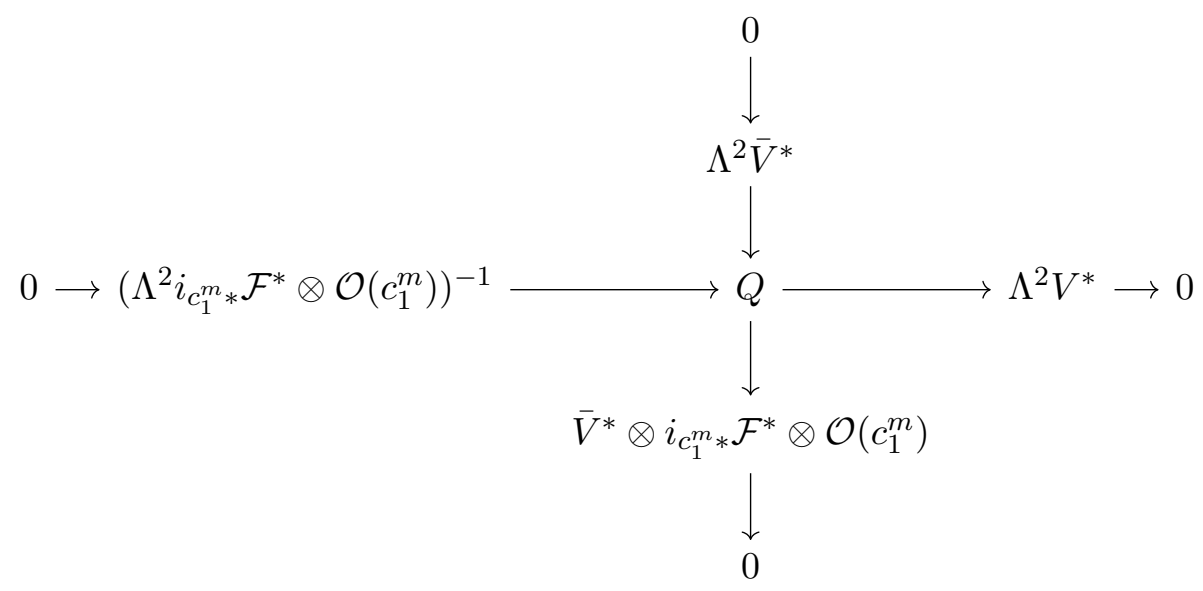

From (4.7) we know that $\Lambda^{2} \bar{V}^{*}=\mathcal{O}_{X}\left(-c_{1}^{m}\right)$. In addition

$$
\Lambda^{2} i_{c_{1}^{m} *} \mathcal{F}^{*} \otimes \mathcal{O}\left(c_{1}^{m}\right)=i_{c_{1}^{m} *} \mathcal{F}^{* \otimes 2} \otimes \mathcal{O}\left(c_{1}^{m}\right)[+1] .
$$

Consequently, the cokernel of $\left\{\left(\Lambda^{2} i_{c_{1}^{m} *} \mathcal{F}^{*} \otimes \mathcal{O}\left(c_{1}^{m}\right)\right)^{-1} \longrightarrow Q\right\}$ will be,

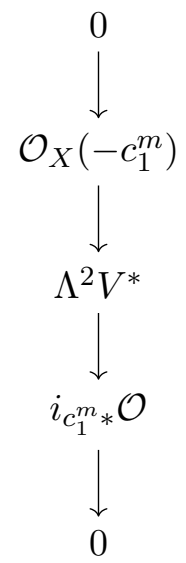

So clearly, $\Lambda^{2} V^{*}=\mathcal{O}_{X}$ as expected. In this case, the result was already known, but we will repeat the same techniques for other higher rank bundles in the following. The idea is basically to add a vertical fiber in the cases that we could compute the $\Lambda^{2} V$ from spectral covers, and check how these vertical components affect the Yukawa couplings.

\subsection{General results for Yukawa couplings}

Let us see how this vertical components can possibly contribute the Yukawa couplings. In general we are looking for a map

$$
H^{1}(V) \otimes H^{1}(V) \longrightarrow H^{2}\left(\Lambda^{2} V\right)=H^{1}\left(\Lambda^{2} V^{*}\right) .
$$

From the way we defined $V$ in (4.8) and the resulting diagram (4.10), one can study the effect of the vertical components. Very roughly speaking the cohomology group $H^{1}(V)$ is made of the following groups

$$
H^{1}(\bar{V}), \quad H^{0}\left(i_{c_{1}^{m} *} \mathcal{F}\right)
$$


and $H^{2}\left(\Lambda^{2} V\right)=H^{1}\left(\Lambda^{2} V^{*}\right)$ are given by,

$$
H^{1}\left(\Lambda^{2} \bar{V}^{*}\right), \quad H^{1}\left(\bar{V}^{*} \otimes i_{c_{1}^{m} *} \mathcal{F}^{*} \otimes \mathcal{O}\left(c_{1}^{m}\right)\right), \quad H^{2}\left(i_{c_{1}^{m} *} \mathcal{F}^{\star \otimes 2} \otimes \mathcal{O}\left(c_{1}^{m}\right)\right) .
$$

Of course, one should check the long exact sequences to see whether these groups actually have non-zero contributions into $H^{1}(V)$ and $H^{2}\left(\Lambda^{2} V\right)$. It is quite possible that one cannot decompose the cohomologies into constituent smaller pieces. For example, there are possible Yukawa couplings from $\bar{V}$,

$$
H^{1}(\bar{V}) \otimes H^{1}(\bar{V}) \longrightarrow H^{1}\left(\Lambda^{2} \bar{V}^{*}\right)=H^{2}\left(\Lambda^{2} \bar{V}\right) .
$$

Such couplings can be found from the results of the previous sections. The new possibilities are,

$$
H^{1}(\bar{V}) \otimes H^{0}\left(i_{c_{1}^{m} *} \mathcal{F}\right) \longrightarrow H^{1}\left(\bar{V} \otimes i_{c_{1}^{m} *} \mathcal{F}\right) \simeq H^{1}\left(\bar{V}^{*} \otimes i_{c_{1}^{m} *} \mathcal{F}^{*} \otimes \mathcal{O}\left(c_{1}^{m}\right)\right)
$$

and

$$
H^{0}\left(i_{c_{1}^{m} *} \mathcal{F}\right) \otimes H^{0}\left(i_{c_{1}^{m} *} \mathcal{F}\right) \longrightarrow H^{0}\left(i_{c_{1}^{m} *} \mathcal{F}^{\otimes 2}\right) \simeq H^{2}\left(i_{c_{1}^{m} *} \mathcal{F}^{* \otimes 2} \otimes \mathcal{O}\left(c_{1}^{m}\right)\right) .
$$

We should add a couple of comments here. First of all the second row is always vanishing. This is related to the smoothness of the bundle $V$. In other words, the cohomology group $H^{2}\left(i_{c_{1}^{m} *} \mathcal{F}^{* \otimes 2} \otimes \mathcal{O}\left(c_{1}^{m}\right)\right)$ does not contribute to $H^{1}\left(\Lambda^{2} V^{*}\right)$. This is because we demand the bundle $V$ and therefore $\Lambda^{2} V$ to be stable and smooth. In this case, the Fourier transform of the bundle should always be WIT-1 (this is a necessary condition for the smoothness plus stability). Also note that for SU(5) models, there are no new $\overline{\mathbf{5}}-\overline{\mathbf{5}}-\mathbf{1 0}$ Yukawa couplings from the vertical components.

So we have the tools for computing the Yukawa couplings for the cases that the spectral cover has a vertical component. In the following these tools are applied for the $E_{6}, \mathrm{SO}(10)$ and $\mathrm{SU}(5)$ models.

\subsection{Bundles with degenerate spectral covers}

In this situation the spectral cover is a degree three non-redced cover with vertical components. We assume that the vertical component is smooth. So the algebraic eaquation for the spectral cover is,

$$
S_{3}=a_{0} Z^{3}
$$

Therefore the spectral sheaf is can be written generally as,

$$
0 \longrightarrow \mathcal{L}_{V} \longrightarrow \mathcal{L}_{3} \longrightarrow \mathcal{L}_{3}^{\prime} \longrightarrow 0
$$

First sheaf is localized over the non-reduced cover $Z^{3}=0$. It can be constructed iteratively as

$$
\begin{aligned}
0 \longrightarrow \mathcal{B}_{1} \longrightarrow \mathcal{L}_{3}^{\prime} \rightarrow \mathcal{L}_{2} \longrightarrow 0 \\
0 \longrightarrow \mathcal{A}_{1} \longrightarrow \mathcal{L}_{2} \rightarrow \mathcal{L}_{1} \longrightarrow 0
\end{aligned}
$$


where $\mathcal{A}_{1}, \mathcal{B}_{1}, \mathcal{L}_{1}$ are line bundles over the zero section $\sigma$, and the extensions of these two sequences are given by,

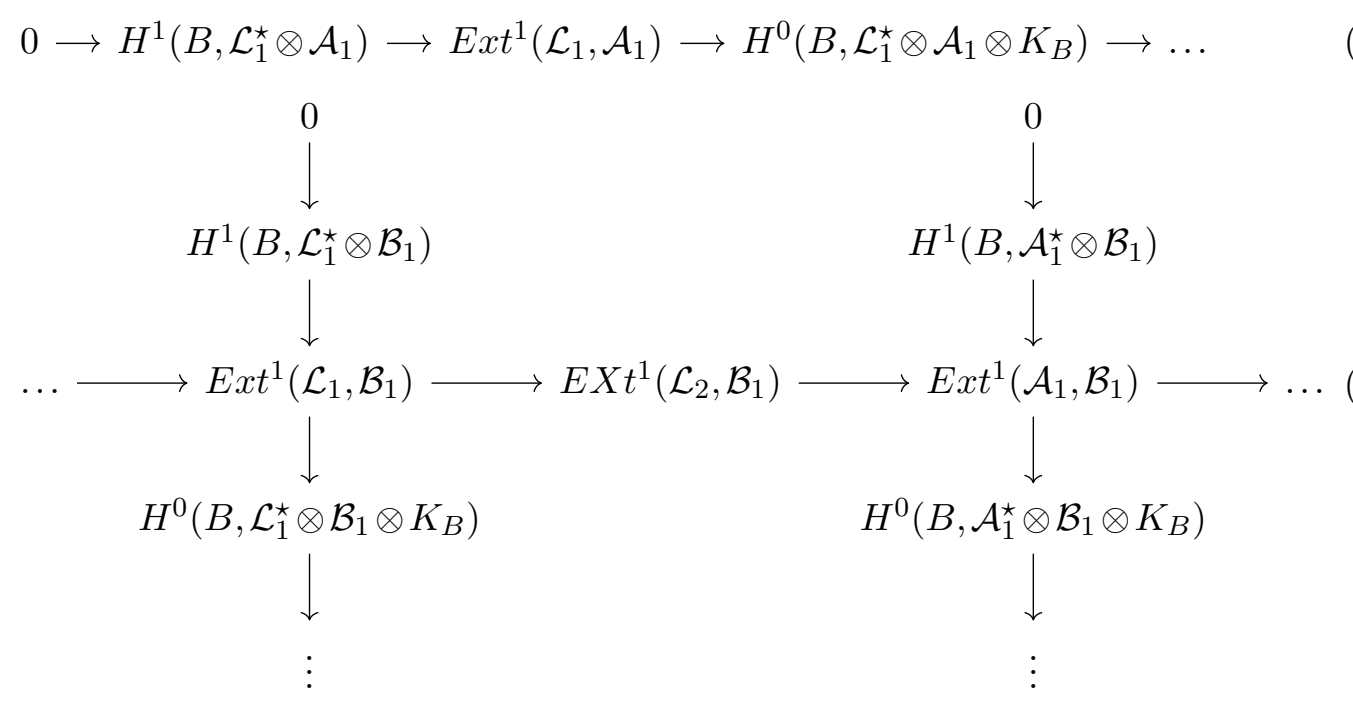

We assume the only terms that are turned on in the extension groups are $H^{0}\left(B, \mathcal{L}_{1}^{\star} \otimes \mathcal{A}_{1} \otimes\right.$ $\left.K_{B}\right), H^{0}\left(B, \mathcal{L}_{1}^{\star} \otimes \mathcal{B}_{1} \otimes K_{B}\right)$ and $H^{0}\left(B, \mathcal{A}_{1}^{\star} \otimes \mathcal{B}_{1} \otimes K_{B}\right)$. Numerical rank of the spectral sheaf is one. ${ }^{23}$ The other groups corresponds to vector bundles (or rank two or three) living over the zero section.

The corresponding vector bundle can be found easily by the inverse Fourier transform,

$$
0 \longrightarrow V_{3} \longrightarrow \bar{V}_{3} \longrightarrow i_{C *} \mathcal{F} \longrightarrow 0, \begin{aligned}
& 0 \longrightarrow \mathcal{B}_{1} \otimes K_{B}^{-1} \longrightarrow \bar{V}_{3} \longrightarrow V_{2} \longrightarrow 0 \\
& 0 \longrightarrow \mathcal{A}_{1} \otimes K_{B}^{-1} \longrightarrow V_{2} \longrightarrow \mathcal{L}_{1} \otimes K_{B}^{-1} \longrightarrow 0
\end{aligned}
$$

First let us take a look at the Yukawa coupling candidates from $\bar{V}_{3}$. We can easily construct $\Lambda^{2} \bar{V}_{3}$

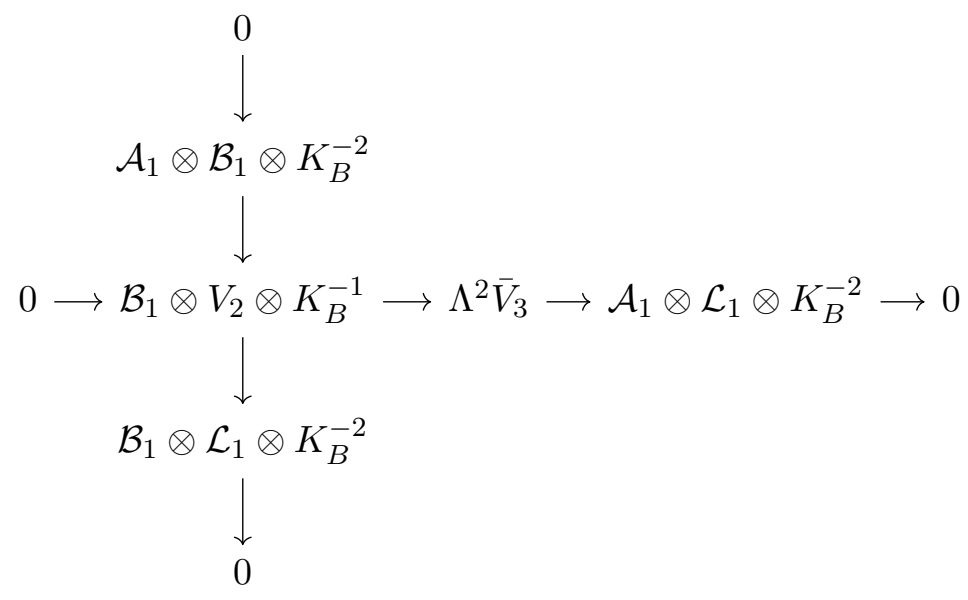

\footnotetext{
${ }^{23}$ By numerical rank one we mean that the Hilbert polynomial of $\mathcal{L}_{3}^{\prime}$ is similar to the Hilbert polynomial of a sine bundle over a smooth triple cover.
} 
Clearly there are three possible Yukawa 27-27-27 couplings from $H^{1}\left(\bar{V}_{3}\right) \otimes H^{1}\left(\bar{V}_{3}\right) \longrightarrow$ $H^{2}\left(\Lambda^{2} \bar{V}_{3}\right)$,

$$
\begin{aligned}
& H^{1}\left(\mathcal{B}_{1} \otimes K_{B}^{-1}\right) \otimes H^{1}\left(\mathcal{L}_{1} \otimes K_{B}^{-1}\right) \longrightarrow H^{2}\left(\mathcal{B}_{1} \otimes \mathcal{L}_{1} \otimes K_{B}^{-2}\right) \\
& H^{1}\left(\mathcal{B}_{1} \otimes K_{B}^{-1}\right) \otimes H^{1}\left(\mathcal{A}_{1} \otimes K_{B}^{-1}\right) \longrightarrow H^{2}\left(\mathcal{A}_{1} \otimes \mathcal{B}_{1} \otimes K_{B}^{-2}\right) \\
& H^{1}\left(\mathcal{A}_{1} \otimes K_{B}^{-1}\right) \otimes H^{1}\left(\mathcal{L}_{1} \otimes K_{B}^{-1}\right) \longrightarrow H^{2}\left(\mathcal{A}_{1} \otimes \mathcal{L}_{1} \otimes K_{B}^{-2}\right)
\end{aligned}
$$

Naively it seems all of the zero modes involving the Yukawa couplings are non-localized in the base manifold $B$. However, note that the extension groups that mentioned above, can kill these zero modes or localize them over curves in $B$. This will be illustrated in the examples. In addition to these couplings, there are other candidates for example, corresponding to the maps in the first row, there are other versions,

$$
\begin{aligned}
& H^{0}\left(\mathcal{B}_{1}\right) \otimes H^{1}\left(\mathcal{L}_{1} \otimes K_{B}^{-1}\right) \longrightarrow H^{1}\left(\mathcal{B}_{1} \otimes \mathcal{L}_{1} \otimes K_{B}^{-1}\right) \\
& H^{1}\left(\mathcal{B}_{1} \otimes K_{B}^{-1}\right) \otimes H^{1}\left(\mathcal{L}_{1}\right) \longrightarrow H^{1}\left(\mathcal{B}_{1} \otimes \mathcal{L}_{1} \otimes K_{B}^{-1}\right)
\end{aligned}
$$

There are similar stories for other vector bundles. Since the vertical components didn't contribute in the $\overline{\mathbf{5}} \overline{\mathbf{5}} \mathbf{- 1 0}$, it is interesting to check to see whether when spectral cover completely degenerates it is possible to have this kind of coupling or not. The analysis is the same as before. Consider only $\bar{V}_{5}$ part in (4.8), and it is simply defined iteratively as the extension of line bundles,

$$
\begin{aligned}
& 0 \longrightarrow \mathcal{W}_{1} \longrightarrow \bar{V}_{5} \longrightarrow V_{4} \longrightarrow 0, \\
& 0 \longrightarrow \mathcal{U}_{1} \longrightarrow V_{4} \longrightarrow V_{3} \longrightarrow 0 \\
& 0 \longrightarrow \mathcal{B}_{1} \longrightarrow V_{3} \longrightarrow V_{2} \longrightarrow 0 \\
& 0 \longrightarrow \mathcal{A}_{1} \longrightarrow V_{2} \longrightarrow \mathcal{L}_{1} \longrightarrow 0
\end{aligned}
$$

and the corresponding sequences for $\Lambda^{2} \bar{V}$ are

$$
\begin{aligned}
& 0 \longrightarrow \mathcal{W}_{1} \otimes V_{4} \longrightarrow \Lambda^{2} \bar{V}_{5} \longrightarrow \Lambda^{2} V_{4} \longrightarrow 0, \\
& 0 \longrightarrow \mathcal{U}_{1} \otimes V_{3} \longrightarrow \Lambda^{2} V_{4} \longrightarrow \Lambda^{2} V_{3} \longrightarrow 0, \\
& 0 \longrightarrow \mathcal{B}_{1} \otimes V_{2} \longrightarrow \Lambda^{2} V_{3} \longrightarrow \mathcal{A}_{1} \otimes \mathcal{B}_{1} \longrightarrow 0 .
\end{aligned}
$$

There is also a relation due to the $c_{1}(V)=0$,

$$
\mathcal{W}_{1} \otimes \mathcal{U}_{1} \otimes \mathcal{B}_{1} \otimes \mathcal{A}_{1} \otimes \mathcal{L}_{1}=K_{B}^{5} \otimes \mathcal{O}\left(c_{V}\right)
$$

One can prove there are candidates for $\overline{\mathbf{5}} \overline{\mathbf{5}} \mathbf{- 1 0}$ using the relation above between the line bundles. We are looking for morphisms $H^{1}(\bar{V}) \otimes H^{1}\left(\Lambda^{2} \bar{V}\right) \longrightarrow H^{2}\left(\Lambda^{3} \bar{V}\right) \simeq H^{1}\left(\Lambda^{2} \bar{V}^{*} \otimes\right.$ $\left.\mathcal{O}\left(c_{V}\right)\right)$. Where in the last isomorphism we used $\Lambda^{5} \bar{V}=\mathcal{O}\left(c_{V}\right)$. Putting everything together one can show for example,

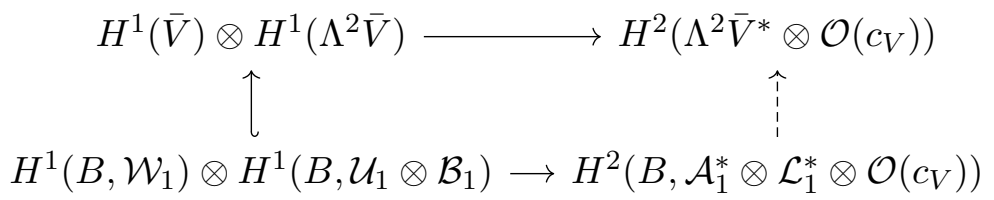


Where the dashed arrow emphasizes that the relation between these two spaces is given by the Leray spectral sequence, and it is not an injection necessarily. There are 15 Yukawa coupling candidates like this one which, as mentioned in the $E_{6}$ model, seems to come from the bulk zero modes inside the 7-branes. However, one needs to check, case by case, whether these zero modes are killed or localized over some curve (by coboundary morphisms or extension maps of the short exact sequences) or not. There are also 30 other candidates. For example, corresponding to the diagram above there are two other diagrams,

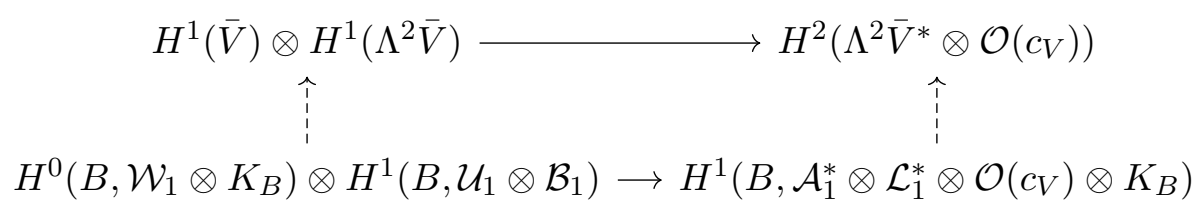

and

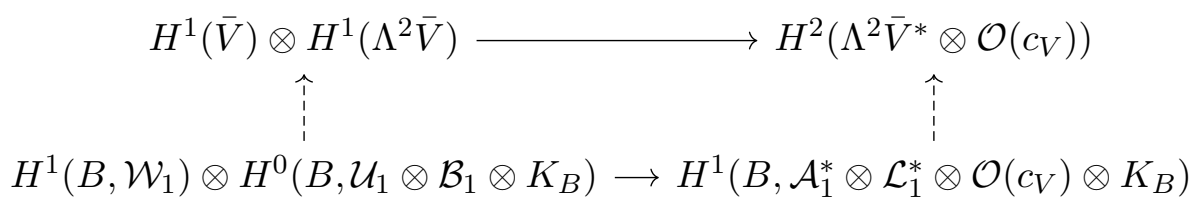

\section{$5 \quad$ Examples}

In this section, we illustrate the ideas of the previous sections by means of simple examples. In this paper we don't try to find models that have the correct phenomenological properties such as correct number of generations, moduli stabilization etc.

\section{$5.1 \quad E_{6}$ model}

For this subsection lets consider a rank three bundle defined as

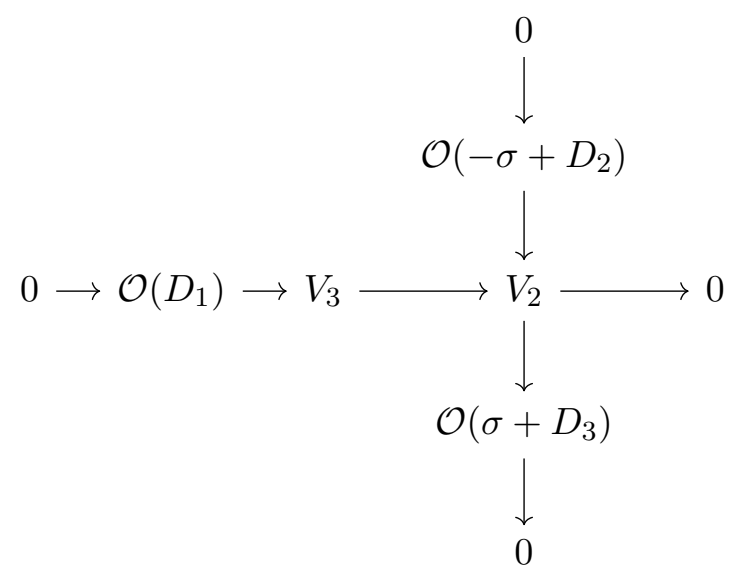

Where $D_{1}, D_{2}, D_{3}$ are divisors in the base that satisfy

$$
D_{1}+D_{2}+D_{3}=0
$$


The Fourier transform of this bundle is quite easy to find,

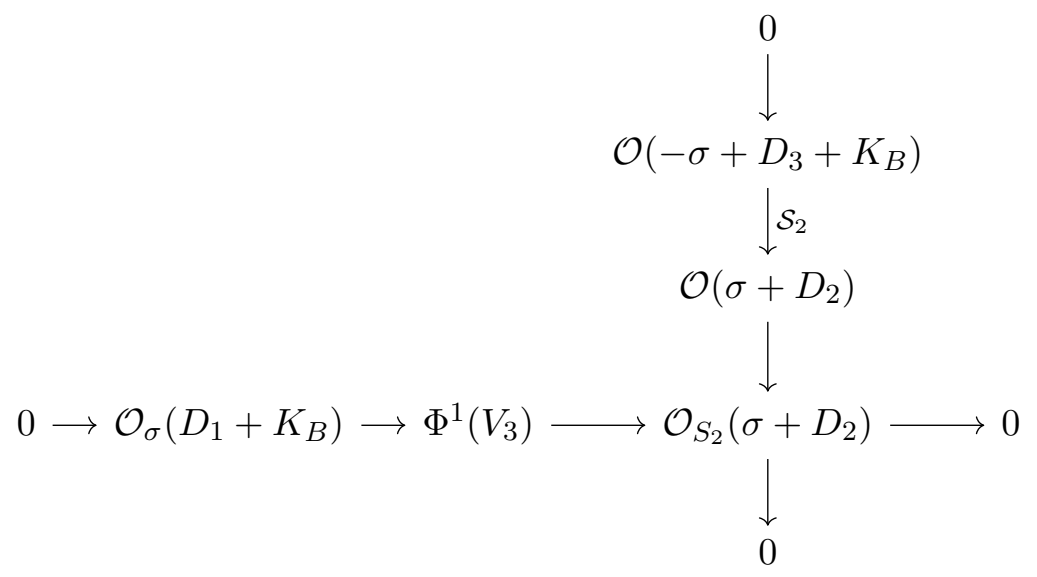

Therefore the spectral cover in this case is

$$
S_{3}=Z \mathcal{S}_{2}=Z\left(a_{2} X+a_{0} Z^{2}\right), \quad\left[\mathcal{S}_{2}\right]=2 \sigma-D_{3}+D_{2}-K_{B}
$$

This is our starting point. The purpose is to compute the Yukawa couplings of this bundle, then consider various deformations of this bundle and see how they affect the Yukawa couplings. The Parameters of this bundle are the polynomials $a_{2}$ and $a_{0}$ which determine the double cover, and the extension group of $V_{2}$ and $\mathcal{O}\left(D_{1}\right)$,

$$
\begin{aligned}
\operatorname{Ext}^{1}\left(\mathcal{O}_{S_{2}}\left(\sigma+D_{2}\right), \mathcal{O}_{\sigma}\left(D_{1}+K_{B}\right)\right) & =H^{0}\left(c, \mathcal{O}\left(D_{1}-D_{2}\right) \otimes \mathcal{O}(c)\right) \\
& =H^{0}\left(c, \mathcal{O}\left(K_{B}+D_{1}-D_{3}\right)\right),
\end{aligned}
$$

where $c$ is the "matter curve" $c=\sigma \cap \mathcal{S}_{2}=K_{B}+D_{2}-D_{3}$.

The first task will be computing the cohomologies and we can just use the results of 3.1.2 with $\mathcal{L}_{1}=\mathcal{O}\left(D_{1}+K_{B}\right)$ and $\mathcal{L}_{2}=\mathcal{O}_{S_{2}}\left(\sigma+D_{2}\right)$. We have seen that there are possible Yukawa coupling in this case from the map

$$
H^{1}\left(\mathcal{L}_{1} \otimes K_{B}^{-1}\right) \otimes H^{0}\left(i_{c *} \mathcal{L}_{2}\right) \longrightarrow H^{1}\left(i_{c *}\left(\mathcal{L}_{1} \otimes \mathcal{L}_{2} \otimes K_{B}^{-1}\right)\right) .
$$

We only need to check whether they actually contribute in the cohmologies $H^{1}(V)$ and $H^{2}\left(\Lambda^{2} V\right)$. Let us start with the former,

$$
\begin{gathered}
\pi_{*} V=\mathcal{O}\left(D_{1}\right) \\
0 \longrightarrow \mathcal{O}\left(D_{1}+K_{B}\right) \longrightarrow \mathcal{O}_{c}\left(D_{2}+K_{B}\right) \longrightarrow 0 .
\end{gathered}
$$

Clearly $H^{1}\left(\mathcal{O}\left(D_{1}\right)\right)$ injects into $H^{1}(V)$. But whether any element in $H^{0}\left(\mathcal{O}_{c}\left(D_{2}+K_{B}\right)\right.$ can be inside $H^{1}(V)$ or not, clearly depends on the choices of the divisors. For example when $B=\mathbb{F}_{0}$ and $D_{1}=(2,-4), D_{2}=(2,9)([c]=(4,12))$ then $h^{*}\left(\mathcal{O}_{c}\left(D_{2}+K_{B}\right)\right)=(8,12)$ and it is indeed part of the $H^{1}(V)$ because $H^{2}\left(\pi_{*} V\right)=0$ in (3.24). In addition the extension of the defining equation is non-zero: $h^{0}\left(c, \mathcal{O}\left(K_{B}+D_{1}-D_{3}\right)\right)=12 .{ }^{24}$

\footnotetext{
${ }^{24}$ It is easy to check that with this choice of divisors there is always a region in Kahler cone such that the slope of $\mathcal{O}\left(D_{1}\right)$ is negative, so the bundle is not semistable.
} 
We also need to check whether $H^{1}\left(i_{c *}\left(\mathcal{L}_{1} \otimes \mathcal{L}_{2} \otimes K_{B}^{-1}\right)\right)=H^{1}\left(c, \mathcal{O}\left(K_{B}+D_{1}+D_{2}\right)\right)$ also maps into $H^{1}\left(R^{1} \pi_{*} \Lambda^{2} V\right)$. It turns out that this is not the case. So there are no $\mathbf{2 7 - 2 7 - 2 7}$ couplings in this case and they are killed by the extension of the defining equation. To see this in more detail remember the long exact sequence that gave us the derived pushforwards of $\Lambda^{2} V$ in section 3.1.2,

$$
\begin{gathered}
0 \longrightarrow \pi_{*} \Lambda^{2} V \longrightarrow \mathcal{O}\left(-D_{1}\right) \longrightarrow \\
\longrightarrow \mathcal{O}_{c}\left(K_{B}+D_{1}+D_{2}\right) \longrightarrow R^{1} \pi_{*} \Lambda^{2} V \longrightarrow \mathcal{O}\left(-D_{1}+K_{B}\right) \longrightarrow 0
\end{gathered}
$$

Note that the coboundary map is exactly the same as the extension of the original defining sequence. So the cokernel of this map is torsion sheaves supported over the points where the extension vanishes i.e., the points where a global section (that one chooses as the extension "parameter") of $\mathcal{O}_{c}\left(K_{B}+D_{1}-D_{3}\right)$ vanishes. So we can conclude

$$
\pi_{*} \Lambda^{2} V=\mathcal{O}\left(-K_{B}+2 D_{3}\right), \quad R^{1} \pi_{*} \Lambda^{2} V=T \oplus \mathcal{O}\left(-D_{1}+K_{B}\right),
$$

where the $T$ is the torsion sheaf mentioned before, and with our choice of the divisors, $T$ is supported over 44 generic points over the curve $c$. So we see that elements of $H^{1}\left(i_{c *}\left(\mathcal{L}_{1} \otimes\right.\right.$ $\left.\mathcal{L}_{2} \otimes K_{B}^{-1}\right)$ ) cannot inject into $H^{2}\left(\Lambda^{2} V\right)$ and these modes are removed by the coboundary map (or extension). So when the extension is chosen to be zero there can be $\mathbf{2 7 - 2 7 - 2 7}$ couplings from localized over $c$.

For $\overline{\mathbf{2 7}} \overline{\mathbf{2 7}}-\overline{\mathbf{2 7}}$ couplings we can use the same techniques as before and we have all information that we need,

$$
\begin{aligned}
H^{1}\left(V^{*}\right) \otimes H^{1}\left(V^{*}\right) & \longrightarrow H^{2}\left(\Lambda^{2} V^{*}\right) \\
H^{2}(V) \otimes H^{2}(V) & \longrightarrow H^{2}(V)
\end{aligned}
$$

The maps above are equivalent. So in this case all we need is just the spectral data of $V$. It is not too hard to show that the relevant cohomologies for this particular coupling are

$$
\begin{aligned}
H^{1}\left(\mathcal{O}\left(D_{1}+K_{B}\right)\right) \otimes H^{1}\left(\mathcal{O}_{c}\left(D_{2}+K_{B}\right)\right) & \simeq H^{1}\left(\mathcal{O}\left(-D_{1}\right)\right) \otimes H^{0}\left(\mathcal{O}_{c}\left(K_{B}-D_{3}\right)\right) \\
& \longrightarrow H^{1}\left(\mathcal{O}_{c}\left(D_{2}+K_{B}\right),\right.
\end{aligned}
$$

where we have used $D_{1}+D_{2}+D_{3}=0$. The only thing we need to show is that whether the elements of $H^{1}\left(\mathcal{O}\left(D_{1}+K_{B}\right)\right)$ enter into $H^{1}\left(R^{1} \pi_{*} V\right)$,

$$
\ldots \longrightarrow H^{0}\left(\mathcal{O}_{c}\left(D_{2}+K_{B}\right)\right) \longrightarrow H^{1}\left(\mathcal{O}\left(D_{1}+K_{B}\right)\right) \longrightarrow H^{1}\left(R^{1} \pi_{*} V\right) \longrightarrow \ldots,
$$

where the second map is simply the extension of the short exact sequence. With the choices of $D_{1}, D_{2}$ and $D_{3}$ in this example, one should find the cokernel of $H^{0}\left(\mathcal{O}_{c}(0,7)\right) \longrightarrow H^{1}(\mathcal{O}(0,-6))$ where the map is an element of $H^{1}\left(\mathcal{O}_{c}(4,-1)\right)$. The basis of these cohomology groups and the generic form of the homomorphism can be represented by (rational) monomials as follows

$$
\begin{array}{ccccc}
X_{1}^{7}, \quad X_{1}^{6} X_{2}, & X_{1}^{5} X_{2}^{2}, & X_{1}^{4} X_{2}^{3}, \quad X_{1}^{3} X_{2}^{4}, & X_{1}^{2} X_{2}^{5}, & X_{1} x_{2}^{6}, \quad X_{2}^{7}, \\
\frac{1}{X_{1} X_{2} X_{1}^{4}}, & \frac{1}{X_{1} X_{2} X_{1}^{3} X_{2}}, \quad \frac{1}{X_{1} X_{2} X_{1}^{2} X_{2}^{2}}, \quad \frac{1}{X_{1} X_{2} X_{1} X_{2}^{3}}, \frac{1}{X_{1} X_{2} X_{2}^{4}}, \\
& \frac{1}{X_{1} X_{2} f_{11}\left(X_{1}, X_{2}\right)},
\end{array}
$$


where the variables $X_{1}$ and $X_{2}$ are homogeneous coordinates of the $\mathbb{P}^{1}$ base of $\mathbb{F}_{0}$, the monomials in the first row are the bases of $H^{0}\left(\mathcal{O}_{c}(0,7)\right)$, the rational monomials of the second row are the bases of $H^{1}(\mathcal{O}(0,-6))$, and the third row is the rational monomial that corresponds to the extension of the defining short exact sequence (with $f_{11}$ being a generic degree 11 polynomial). It is now very easy to show that this map is surjective, in other words $H^{1}\left(\mathcal{O}\left(D_{1}+K_{B}\right)\right)$ do not inject into $H^{1}\left(R^{1} \pi_{*} V\right)$ and hence no contribution to $H^{2}(V)$. So there is no $\overline{\mathbf{2 7}}-\overline{\mathbf{2 7}}-\overline{\mathbf{2 7}}$ coupling in this model. Here we clearly see one of the main points of this paper. Instead of computing the Yukawa couplings by directly computing the cohomology groups of $V$, one can dissect this problem into smaller pieces using the spectral data, reduce the problem to simple calculations.

Before considering the deformations, it is somewhat important to mention that there is another Yukawa coupling, namely $\mathbf{1 - 2 7 - \overline { 2 7 }}$, which can be non-zero. This is given by the Yoneda pairing,

$$
\operatorname{Ext}^{1}(\mathcal{O}, V) \otimes \operatorname{Ext}^{1}(V, V) \otimes \operatorname{Ext}^{1}(V, \mathcal{O}) \longrightarrow \operatorname{Ext}^{3}(\mathcal{O}, \mathcal{O})=H^{3}(\mathcal{O})=\mathbb{C}
$$

The middle term in this pairing counts the infinitesimal deformations of the bundle. In the current example they correspond to the deformations of $\mathcal{S}_{2}$ and the extension group elements. When the bundle moduli are stabilized to some fixed values, these couplings give mass to the 27- $\overline{\mathbf{2 7}}$ pairs but the net number of generations remains unchanged. Anyways, in this example these couplings are non-zero and are localized over the curve $c$ and they are counted by the following map

$$
H^{0}\left(\mathcal{O}_{c}\left(K_{B}+D_{2}\right)\right) \otimes H^{0}\left(\mathcal{O}_{c}\left(K_{B}+D_{1}-D_{3}\right)\right) \otimes H^{1}\left(\mathcal{O}\left(-D_{1}\right)\right) \longrightarrow H^{1}\left(c, K_{c}\right) \simeq \mathbb{C}
$$

With the choices we have made all of the cohomologies above are non-zero. But the previous calculations show that $H^{1}\left(\mathcal{O}\left(-D_{1}\right)\right)$ doesn't contribute to $H^{2}\left(\mathcal{O}\left(-D_{1}\right)\right)$. Therefore this coupling is also vanishing.

In the next part we deform the spectral data to a completely degenerate one by setting the polynomial $a_{2}$ to zero. The spectral sheaf can be rewritten in the form of the previous subsection,

$$
0 \longrightarrow \mathcal{L}_{c_{V}} \longrightarrow \mathcal{L}_{3} \longrightarrow \overline{\mathcal{L}}_{3} \longrightarrow 0
$$

It is possible to find $\mathcal{L}_{V}$ and $\overline{\mathcal{L}}_{3}$ by computing $L i_{c_{V}}^{*} \mathcal{L}_{3}$ and $L i_{\sigma}^{*} \mathcal{L}_{3}$ using two different representations of $\mathcal{L}_{3}$, and compare them. The final result is,

$$
\begin{gathered}
\mathcal{L}_{c_{V}}=\mathcal{O}_{c_{V}}\left(-2 \sigma+D_{2}\right), \\
0 \longrightarrow \overline{\mathcal{O}}_{\sigma}\left(-2 D_{3}\right) \longrightarrow \overline{\mathcal{L}}_{2} \longrightarrow 0 \\
0 \longrightarrow \mathcal{\mathcal { L }}_{\sigma}\left(D_{2}\right) \longrightarrow \overline{\mathcal{L}}_{2} \longrightarrow \mathcal{O}_{\sigma}\left(D_{2}+K_{B}\right) \longrightarrow 0
\end{gathered}
$$

All of the extension morphisms are non-zero and generic. As usual, instead of working 
with the vertical components directly, one can perform an inverse Fourier transform,

$$
\begin{aligned}
& 0 \longrightarrow V_{3} \longrightarrow \bar{V}_{3} \longrightarrow i_{c_{V} *} \mathcal{F} \longrightarrow 0 \\
& 0 \longrightarrow \mathcal{O}_{c_{V}}\left(-4 \sigma+D_{2}+K_{B}\right) \\
& \quad \longrightarrow\left(K_{B} \oplus K_{B}^{-1} \oplus K_{B}^{-2}\right) \otimes \mathcal{O}_{c_{V}}\left(-\sigma+D_{2}\right) \longrightarrow i_{c_{V} *} \mathcal{F} \longrightarrow 0
\end{aligned}
$$

To do concrete calculations one needs to choose the divisors $D_{1}, D_{2}$ and $D_{3} \cdot{ }^{25}$ We choose $D_{1}=(2,-4)$ and $D_{2}(2,3)$. Then $D_{3}=(-4,1)$ and $c_{V}=(8,4)$. In this case one can show the cohomologies of $i_{c_{V} *} \mathcal{F}=(60,0,0,0)$. Pushforwads are also easy to find,

$$
\begin{aligned}
& 0 \longrightarrow \mathcal{O}\left(-2 D_{3}-K_{B}\right) \longrightarrow \pi_{*} V \longrightarrow \mathcal{O}\left(D_{2}-K_{B}\right) \longrightarrow 0 \\
& 0 \longrightarrow \mathcal{O}\left(-2 D_{3}\right) \longrightarrow \pi_{*} V \longrightarrow \mathcal{O}\left(D_{2}+K_{B}\right) \longrightarrow 0
\end{aligned}
$$

In principle, between the first row and the second row is non-zero, but in this example it is vanishing. By the analysis of the previous section, especially the relations (4.26), there are only three "types" of candidates for the Yukawa couplings from the degenerate part of the spectral cover

$$
\begin{aligned}
H^{1}\left(\mathcal{B}_{1} \otimes K_{B}^{-1}\right) \otimes H^{1}\left(\mathcal{A}_{1} \otimes K_{B}^{-1}\right) & \longrightarrow H^{2}\left(\mathcal{B}_{1} \otimes \mathcal{A}_{1} \otimes K_{B}^{-2}\right), \\
H^{0}\left(\mathcal{B}_{1}\right) \otimes H^{1}\left(\mathcal{A}_{1} \otimes K_{B}^{-1}\right) & \longrightarrow H^{1}\left(\mathcal{B}_{1} \otimes \mathcal{A}_{1} \otimes K_{B}^{-1}\right), \\
H^{1}\left(\mathcal{B}_{1} \otimes K_{B}^{-1}\right) \otimes H^{0}\left(\mathcal{L}_{1}\right) & \longrightarrow H^{1}\left(\mathcal{B}_{1} \otimes \mathcal{A}_{1} \otimes K_{B}^{-1}\right),
\end{aligned}
$$

where $\mathcal{B}_{1}=\mathcal{O}\left(-2 D_{3}\right), \mathcal{A}_{1}=\mathcal{O}\left(D_{2}\right)$ and $\mathcal{L}_{1}=\mathcal{O}\left(D_{2}+K_{B}\right)$. One needs to check whether these cohomologies can contribute to $H^{1}(V)$ and $H^{2}\left(\Lambda^{2} V\right)$. Since the cohomologies of $\mathcal{F}$ are $(60,0,0)$, any element of $H^{1}(\bar{V})$ can be lifted to an element in $H^{1}(V)$. However, all of the relevant cohomologies of the line bundles $\mathcal{A}_{1} \mathcal{B}_{1}$ and $\mathcal{L}_{1}$, that can feed non-zero elements into $H^{1}(\bar{V})$, and at the same time give non-zero Yukawa couplings, are vanishing. Therefore there is no $\mathbf{2 7 - 2 7 - 2 7}$ couplings of this type.

The final part is checking for the couplings induced by the vertical component of the spectral cover. The candidates are given in the relations (4.17) and (4.18). The cohomologies of $i_{c_{V} *} \mathcal{F}^{* \otimes 2} \otimes \mathcal{O}\left(c_{V}\right)$ are $(0,824,0)$. Therefore the couplings corresponding to (4.18) are zero, consistent with our expectation. For the other option one gets $h^{1}(\bar{V})=$ 2 which is lifted from $h^{1}\left(\mathcal{O}\left(D_{2}\right)\right)$, and $h^{0}\left(i_{c_{V} *} \mathcal{F}\right)=60$. However one can check easily $h^{1}\left(i_{c_{V} *} \mathcal{F} \otimes \bar{V}\right)=0$. So nothing left and all of the 27-27-27 couplings are zero.

\footnotetext{
${ }^{25}$ With the previous choices the bundle becomes unstable as in the limit where the spectral cover is degenerate.
} 


\section{$5.2 \mathrm{SU}(5)$ model}

We consider a vector bundle defined by (3.129),

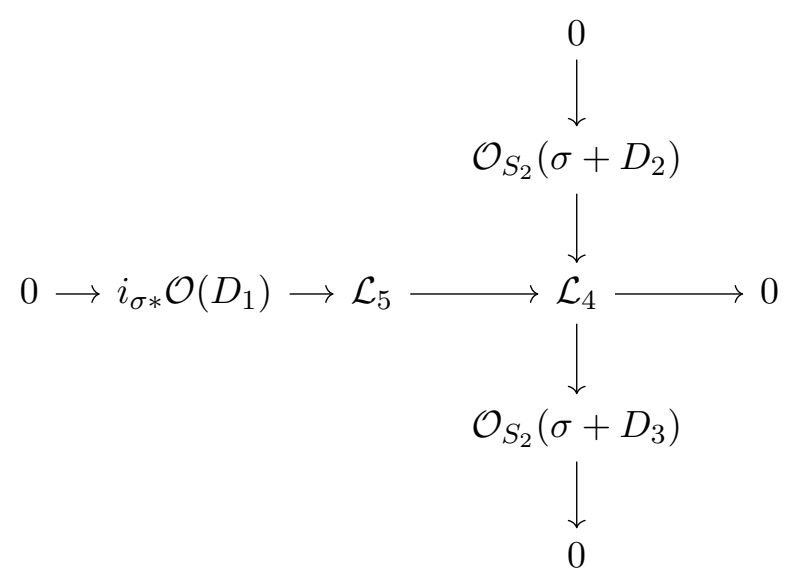

where $S_{2}$ is a double cover with divisor class $\left[S_{2}\right]=2 \sigma+\eta$. We have shown that there is a 1010-5 coupling candidate (3.132), and three candidates for $\overline{\mathbf{5}} \overline{\mathbf{5}} \mathbf{- 1 0}$ (3.135), (3.136), (3.137). We only need to check whether in a certain model the cohomologies in the couplings are non-zero, they contribute to the relevant cohomologies of $V$ and $\Lambda^{2} V$. We need a bundle $V$ which is stable and degree zero. For the latter condition, $\operatorname{Det}\left(\pi_{*} \mathcal{L}_{5}\right)=K_{B}^{5}$. This puts a constraint between $\eta, D_{1}, D_{2}$ and $D_{3}$. To find this one should compute the pushforward of $\pi_{*} \mathcal{O}_{S_{2}}(\sigma)$ first,

$$
0 \longrightarrow \mathcal{O} \longrightarrow \pi_{*} \mathcal{O}_{S_{2}}(\sigma) \longrightarrow \mathcal{O}\left(K_{B}-\eta\right) \longrightarrow 0
$$

Therefore,

$$
\operatorname{Det}\left(\pi_{*} \mathcal{O}_{S_{2}}\left(\sigma+D_{2}\right)\right)=\mathcal{O}\left(K_{B}-\eta+2 D_{3}\right), \quad \operatorname{Det}\left(\pi_{*} \mathcal{O}_{S_{2}}\left(\sigma+D_{2}\right)\right)=\mathcal{O}\left(K_{B}-\eta+2 D_{3}\right) .
$$

Deriving the final result is now easy,

$$
D_{1}+2 D_{2}+2 D_{3}-2 \eta=3 K_{B} .
$$

On the other hand, the stability requires $h^{0}(V)=h^{3}(V)=0$. So combining (3.128) with the Leray spectral sequence, one gets

$$
H^{0}(V)=H^{0}\left(\pi_{*} V\right), \quad H^{3}(V)=H^{2}\left(R^{1} \pi_{*} V\right) .
$$

The following choice satisfies these conditions,

$$
B=\mathbb{F}_{1}, \quad D_{1}=(-4,3), \quad D_{2}=(3,3), \quad D_{3}=(1,2) \Rightarrow \eta=(5,11) .
$$

With this choice, the extension of the vertical column can be computed as usual, ${ }^{26}$

$$
\begin{aligned}
\operatorname{RHom}\left(i_{S_{2} *} \mathcal{O}\left(\sigma+D_{3}\right), i_{S_{2} *} \mathcal{O}\left(\sigma+D_{2}\right)\right) & =R \Gamma\left(\mathcal{O}_{S_{2}}\left(D_{2}-D_{3}\right) \otimes\left(\mathcal{O} \oplus \mathcal{O}\left(S_{2}\right)[-1]\right)\right) \\
& \Downarrow \\
0 \operatorname{Ext}_{X}^{1}\left(\mathcal{O}_{S_{2}}\left(\sigma+D_{3}\right), \mathcal{O}_{S_{2}}\left(\sigma+D_{2}\right)\right) & \longrightarrow H^{0}\left(\mathcal{O}_{S_{2}}\left(S_{2}+D_{2}-D_{3}\right)\right) \\
& \longrightarrow H^{2}\left(\mathcal{O}_{S_{2}}\left(D_{2}-D_{3}\right)\right) \longrightarrow \ldots
\end{aligned}
$$

\footnotetext{
${ }^{26} \Gamma$ is the global section functor.
} 
It is not hard to show $h^{0}\left(\mathcal{O}_{S_{2}}\left(S_{2}+D_{2}-D_{3}\right)\right)=95$ and $h^{2}\left(\mathcal{O}_{S_{2}}\left(D_{2}-D_{3}\right)\right)=39$. So there are at least 56 independent extension morphisms that can give a "line bundle" over the degree four cover $S_{2}^{2}$ (as opposed to a rank two bundle over $S_{2}$ ).

To have non-zero Yukawa couplings, $H^{1}\left(B, \mathcal{O}\left(D_{1}-K_{B}\right)\right)$ must be non-empty. The cohmologies of this line bundle are $(0,8,0)$. Together with the Leray spectral sequence,

$$
0 \rightarrow H^{1}\left(\mathcal{O}\left(D_{1}\right) \otimes K_{B}^{-1}\right) \longrightarrow H^{1}(V) \rightarrow H^{0}\left(R^{1} \pi_{*} V\right) \rightarrow 0
$$

it is clear that the elements of $H^{1}\left(B, \mathcal{O}\left(D_{1}-K_{B}\right)\right)$ injects into $H^{1}(V)$. What about $H^{0}\left(R^{1} \pi_{*} V\right)$ ? For Yukawa couplings one needs to check whether at least some elements of $\left.\mathcal{L}_{4}\right|_{c}$ (where $c=S_{2} \cdot \sigma$ ) can lift into some non-zero elements in $H^{0}\left(R^{1} \pi_{*} V\right.$ ). Recall the following diagram,

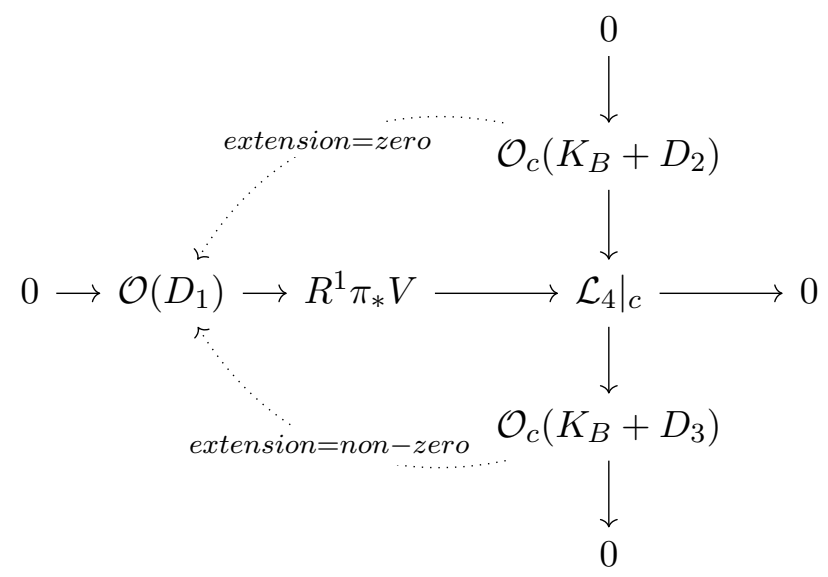

The extension of the sequence that defines $R^{1} \pi_{*} V$ is non-zero, and in principle it comes from two pieces one piece $\mathcal{O}_{c}\left(K_{B}+D_{2}\right)$ and one from $\mathcal{O}_{c}\left(K_{B}+D_{3}\right)$. With this information we can show that $h^{*}\left(R^{1} \pi_{*} V\right)=(5,22,0)$, and all of the five independent elements of $h^{0}\left(R^{1} \pi_{*} V\right)$ are coming from $\left.\mathcal{L}_{4}\right|_{c}$. More precisely,

$$
\begin{aligned}
& h^{*}\left(\mathcal{O}\left(D_{1}\right)\right) \quad h^{*}\left(R^{1} \pi_{*} V\right) \quad h^{*}\left(\left.\mathcal{L}_{4}\right|_{c}\right) \\
& 0 \quad 5 \quad 5 \leftrightarrow h^{0}\left(\mathcal{O}_{c}\left(K_{B}+D_{2}\right)\right) \\
& \text { extension }=\text { zero } \\
& 18<4 \leftrightarrow h^{1}\left(\mathcal{O}_{c}\left(K_{B}+D_{3}\right)\right) \\
& \begin{array}{lll}
0 & 0 & 0
\end{array}
\end{aligned}
$$

So far we have identified 40 possible 10-10-5 couplings from $H^{1}\left(B, \mathcal{O}\left(D_{1}-K_{B}\right)\right) \otimes$ $H^{0}\left(c, \mathcal{O}_{c}\left(D_{2}+K_{B}\right)\right)$. To make sure they are actually non-zero the cohomologies of $\Lambda^{2} V$ are required. The generalities are explained before in previous sections, here we only add some of the details. Remeber we had the following short exact sequence,

$$
0 \longrightarrow \mathcal{O}\left(D_{1}\right) \otimes \mathcal{L}_{4} \longrightarrow \mathcal{L}_{5} \star_{A} \mathcal{L}_{5} \longrightarrow \mathcal{L}_{4} \star_{A} \mathcal{L}_{4} \longrightarrow 0 .
$$


The first term on the right is hard to compute. But it is already done before,

$$
\begin{aligned}
\mathcal{L}_{4} \star_{A} \mathcal{L}_{4} & =i_{\sigma *} \operatorname{Det}\left(\pi_{*} \mathcal{O}_{S_{2}}(\sigma)+D_{2}\right) \oplus i_{\sigma *} \operatorname{Det}\left(\pi_{*} \mathcal{O}_{S_{2}}(\sigma)+D_{3}\right) \oplus \mathcal{L}_{2} \star \mathcal{L}_{2}^{\prime} \\
& =\mathcal{O}_{\sigma}\left(K_{B}-\eta+2 D_{2}\right) \oplus \mathcal{O}_{\sigma}\left(K_{B}-\eta+2 D_{3}\right) \oplus \mathcal{L}_{2} \star \mathcal{L}_{2}^{\prime}
\end{aligned}
$$

We only need to compute the last term,

$$
\mathcal{L}_{2} \star \mathcal{L}_{2}^{\prime}=\mathcal{O}\left(D_{2}+D_{3}\right) \otimes \mathcal{O}_{S_{2}}(\sigma) \star \mathcal{O}_{S_{2}}(\sigma) .
$$

Here we give a rather heuristic, but direct way, and in the appendix we prove this formula indirectly by using inverse Fourier transform. It has been shown that the antisymmetric part of this product is simply the determinant of the pushforward. For general product we know the product should be supported over a degree four but reducible cover, which one component of that is a non-reduced degree two copy of the zero section. The definition of the Pontrjagin product helps to derive a formula for the restriction of $\mathcal{O}_{S_{2}}(\sigma) \star \mathcal{O}_{S_{2}}(\sigma)$ on the zero section,

$$
r: \mathcal{O}_{S_{2}}(\sigma) \star \mathcal{O}_{S_{2}}(\sigma) \longrightarrow \pi_{*}\left(\mathcal{O}_{S_{2}}(\sigma) \otimes \tau^{*} \mathcal{O}_{S_{2}}(\sigma)\right),
$$

where $\tau$ is the involution of the elliptic fibers $(y \rightarrow-y)$. Since any double cover and also the divisor $\sigma$ are invariant under the involution then $\tau^{*} \mathcal{O}_{S_{2}}(\sigma)=\mathcal{O}_{S_{2}}(\sigma)$. Therefore we can compute the restriction over zero section,

$$
\begin{gathered}
r\left(\mathcal{O}_{S_{2}}(\sigma) \star \mathcal{O}_{S_{2}}(\sigma)\right)=\pi_{*} \mathcal{O}_{S_{2}}(2 \sigma), \\
0 \longrightarrow \mathcal{O}\left(2 K_{B}+\eta\right) \longrightarrow \pi_{*} \mathcal{O}_{S_{2}}(2 \sigma) \longrightarrow \mathcal{O}\left(K_{B}-\eta\right) \longrightarrow 0 .
\end{gathered}
$$

Note that the last term on the right is just the antisymmetric part of the Pontrjagin product, i.e., $\operatorname{Det}\left(\pi_{*} \mathcal{O}_{S_{2}}(\sigma)\right)=\mathcal{O}_{S_{2}}(\sigma) \star_{A} \mathcal{O}_{S_{2}}(\sigma)$. So the kernel should be the restriction of the symmetric product restricted over the fiber. ${ }^{27}$ So we expect $\mathcal{O}_{S_{2}}(\sigma) \star \mathcal{O}_{S_{2}}(\sigma)$ can be represented by the following diagram,

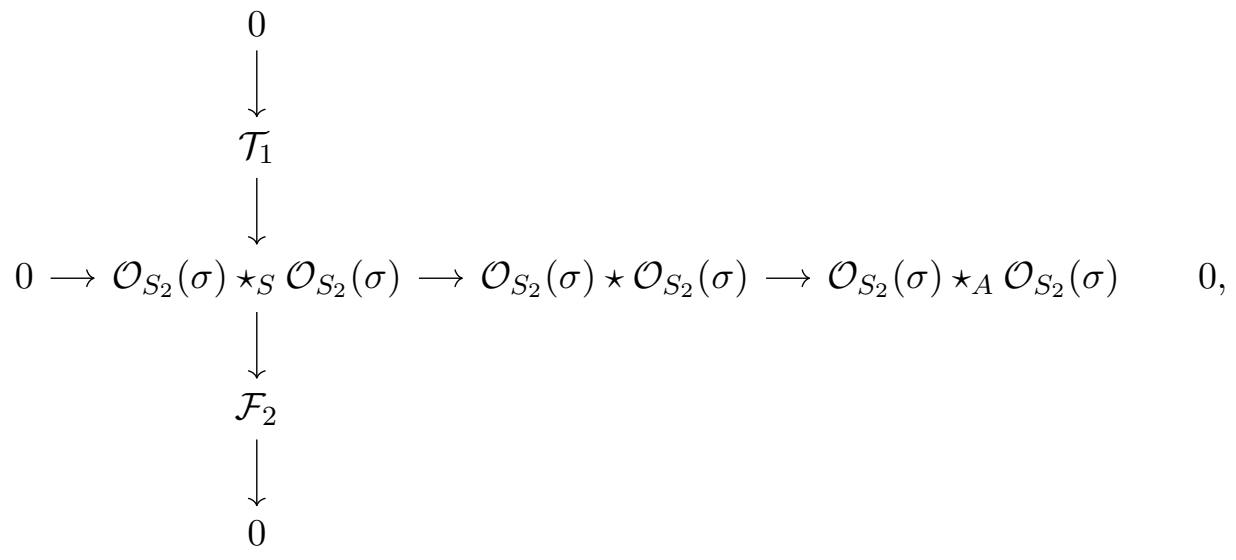

\footnotetext{
${ }^{27}$ This can also be seen by noting that the two terms differ by $\frac{B r}{2}$ where $B r=4 \eta+2 K_{B}$ is the branching divisor of $S_{2}$ [95].
} 
where $\mathcal{T}_{1}$ is a line bundle over the zero section to be determined soon, $\mathcal{F}_{2}$ is a line bundle over the double cover mentioned in sec ??. As emphasized before, we expect that $i_{\sigma}^{*} \mathcal{O}_{S_{2}}(\sigma) \star_{S}$ $\mathcal{O}_{S_{2}}(\sigma)=\mathcal{O}\left(2 K_{B}+\eta\right) .{ }^{28}$ To determine $\mathcal{T}_{1}$ note that the zero section and the support of $\mathcal{F}_{2}$ intersect on the branching divisor of $S_{2}$. Therefore we expect that

$$
\mathcal{T}_{1}=\mathcal{O}\left(2 K_{B}+\eta\right) \otimes \mathcal{O}(-B r)=\mathcal{O}(-3 \eta)
$$

So the final result about $L i_{\sigma}^{*} \mathcal{L}_{2} \star \mathcal{L}_{2}^{\prime}$ can be derived easily,

$$
\begin{aligned}
0 & \longrightarrow \mathcal{O}\left(-3 \eta+D_{2}+D_{3}-K_{B}\right) \longrightarrow L^{-1} i_{\sigma}^{*} \mathcal{L}_{2} \star \mathcal{L}_{2}^{\prime} \longrightarrow \mathcal{O}\left(-\eta+D_{2}+D_{3}\right) \\
& \longrightarrow \mathcal{O}\left(\eta+D_{2}+D_{3}+2 K_{B}\right) \longrightarrow i_{\sigma}^{*} \mathcal{L}_{2} \star \mathcal{L}_{2}^{\prime} \longrightarrow \mathcal{O}\left(-\eta+D_{2}+D_{3}+K_{B}\right) \longrightarrow 0 .
\end{aligned}
$$

A comment on the coboundary maps is useful at this point. Note that the coboundary map of the sequence cannot be induced by the Ext groups of the original sequence that defined $\mathcal{L}_{4}$ for us. Similarly, same thing happens for the maps between $\operatorname{Det} \pi_{*} \mathcal{L}_{2}$ and $\mathcal{L}_{2}^{\prime}$ and other components of $\mathcal{L}_{4} \star_{A} \mathcal{L}_{4}$.

Now everything for computing $L i_{\sigma}^{*} \mathcal{L}_{5} \star_{A} \mathcal{L}_{5}$ is ready,

$$
\begin{gathered}
\left.0 \longrightarrow \pi_{*} \Lambda^{2} V \longrightarrow \begin{array}{c}
\mathcal{O}\left(-K_{B}-\eta+2 D_{2}\right) \oplus \mathcal{O}\left(-K_{B}-\eta+2 D_{3}\right) \\
\oplus \mathcal{O}\left(-2 K_{B}-3 \eta+D_{2}+D_{3}\right) \oplus \mathcal{O}\left(-K_{B}-\eta+D_{2}+D_{3}\right)
\end{array}\right] \\
\left.\rightarrow \mathcal{O}\left(D_{1}\right) \otimes \mathcal{L}_{4}\right|_{c} \longrightarrow R^{1} \pi_{*} \Lambda^{2} V \longrightarrow \begin{array}{c}
\mathcal{O}\left(-\eta+2 D_{2}\right) \oplus \mathcal{O}\left(-\eta+2 D_{3}\right) \\
\oplus \mathcal{O}\left(\eta+D_{2}+D_{3}+K_{B}\right) \oplus \mathcal{O}\left(-\eta+D_{2}+D_{3}\right)
\end{array}
\end{gathered}
$$

Even though the cokernel of this long exact sequence is non-zero, with the current choices of $D_{1}, D_{2}$ and $D_{3}$ all of the cohomologies of $\left.\mathcal{W}_{1} \otimes \mathcal{L}_{4}\right|_{c} \otimes K_{B}^{-1}=\mathcal{O}_{c}\left(D_{1}-K_{B}\right) \otimes \mathcal{L}_{4}$ actually inject into the cohomologies of $R^{1} \pi_{*} \Lambda^{2} V$. In particular, for the 10-10-5 coupling only $H^{1}\left(R^{1} \pi_{*} \Lambda^{2} V\right)$ is needed (3.132), and by Leray sequence all the elements of this cohomology group lift into $H^{2}\left(\Lambda^{2} V\right)$. However, the relevant cohomologies of $\operatorname{Det}\left(\pi_{*} \mathcal{L}_{2}\right)$ and $\operatorname{Det}\left(\pi_{*} \mathcal{L}_{2}^{\prime}\right)$ in (3.135) and (3.136) are zero. So there are no $\overline{\mathbf{5}} \mathbf{- 5}-\mathbf{- 1 0}$ couplings in this theory. To check whether the 10-10-5 couplings actually exist in the effective theory, one needs to check whether the elements of $H^{1}\left(\mathcal{O}\left(D_{1}-K_{B}\right)\right)$ and $H^{0}\left(\left.\mathcal{L}_{4}\right|_{c}\right)$ can "contract" with each other and give an element of $H^{1}\left(\mathcal{O}_{c}\left(D_{1}-K_{B}\right) \otimes \mathcal{L}_{4}\right)$. Luckily in this example we can say that without any calculations. Recall that $H^{0}\left(\left.\mathcal{L}_{4}\right|_{c}\right)$ has five independent elements which are injected from $H^{0}\left(\mathcal{O}_{c}\left(K_{B}+D_{2}\right)\right)$. So to check whether the map in (3.132) is non-zero, we should examine whether the following map is non-zero

$$
H^{1}\left(\mathcal{O}\left(D_{1}-K_{B}\right)\right) \otimes H^{0}\left(\mathcal{O}_{c}\left(D_{2}+K_{B}\right)\right) \stackrel{?}{\rightarrow} H^{1}\left(\mathcal{O}_{c}\left(D_{1}+D 2\right)\right) \rightarrow H^{1}\left(\mathcal{O}_{c}\left(D_{1}-K_{B}\right) \otimes \mathcal{L}_{4}\right) .
$$

It tuns out $h^{1}\left(\mathcal{O}_{c}\left(D_{1}+D_{2}\right)\right)=0$. So there is no 10-10-5 couplings in this effective theory either.

\footnotetext{
${ }^{28}$ Of course $i_{\sigma}^{*} \mathcal{O}_{S_{2}}(\sigma) \star_{S} \mathcal{O}_{S_{2}}(\sigma)=\mathcal{O}\left(2 K_{B}+\eta\right)=\mathcal{O}\left(K_{B}-\eta\right)$
} 


\section{Elliptic fibrations without holomorphic section}

So far, we assumed the Calabi-Yau threefold is given by the Weierstrass model. As long as the elliptic fibration has a holomorphic (in other words, the section is isomorphic to the base manifold), it is easy to generalize these results. Because to define the Poincare sheaf, the section of the fibration should hit every fiber at exactly one point. However, most of the elliptically fibered Calabi-Yau threefolds do not have any holomorphic sections. In these cases if someone defines an integral transform with kernel chosen to be the "naive" Poincare sheaf $\mathcal{I}_{\Delta} \otimes \pi_{1}^{*} \mathcal{O}(\sigma) \otimes \pi_{2}^{*} \mathcal{O}(\sigma)$, then that integral transform is not a Fourier-Mukai transform anymore.

It is very important for phenomenological applications to generalize the approach presented in the previous sections such that they can be used for the non-holomorphic section too. We only need to find the right kernel such that when the transform is restricted to any elliptic fiber (reducible or not) is reduced to the ordinary Fourier-Mukai transform.

Here we only briefly propose a solution for case the reducible fibers are $I_{2}$. This approach can be generalized for $I_{n}$ fibers. However the for other types of reducible elliptic fibers, one needs more sophisticated approaches.

First note that for a single elliptic curve, reducible or not, the Poincare sheaf always defines a Fourier-Mukai transform. The reason is one can always choose the zero section to be a point on the curve. For smooth elliptic curves it is well known, and for $I_{2}$ fibers it is proved by Caldararu [97]. The problem appears when one considers an elliptic fibration where both types of fibers can appear in one family. When the section of the family is rational, the restriction of $\mathcal{I}_{\Delta} \otimes \pi_{1}^{*} \mathcal{O}(\sigma) \otimes \pi_{2}^{*} \mathcal{O}(\sigma)$ over smooth fibers is the Poincare sheaf, but its restriction over $I_{2}$ fibers (if the section wraps around one component, say $c_{1}$ ) is not. More precisely, suppose $i_{p}: E_{p} \hookrightarrow X$ be the inclusion morphism of the fiber over the point $p \in B$. If $E_{p}$ is $I_{2}$ and $\sigma$ is the rational section that wraps around $c_{1}$, then the restriction over $E_{p}$ is given by,

$$
0 \longrightarrow i_{p}^{*} \mathcal{O}_{X}(\sigma) \longrightarrow \mathcal{O}_{c_{1}}(-1) \oplus \mathcal{O}_{c_{2}}(+2) \longrightarrow T \longrightarrow 0
$$

where $T$ is a torsion sheaf supported over the intersection points of $c_{1}$ and $c_{2}$. However, a true section that hits a point in $c_{1}$ (denoted by $\sigma_{0}$ ) can be represented as

$$
0 \longrightarrow \mathcal{O}_{E_{p}}\left(\sigma_{0}\right) \longrightarrow \mathcal{O}_{c_{1}}(+1) \oplus \mathcal{O}_{c_{2}} \longrightarrow T \longrightarrow 0
$$

Ideally, if one can modify $\mathcal{O}(\sigma)$ such that over smooth fibers it is just the ordinary section that hits a single point, i.e., the modification has no effect over smooth fibers, but over the $I_{2}$ fibers it modifies $i_{p}^{*} \mathcal{O}_{X}(\sigma)$ to $\mathcal{O}_{E_{p}}\left(\sigma_{0}\right)$ then we reach our purpose. The way we have been able to modify $\mathcal{O}(\sigma)$, turns the restriction on the reducible elliptic fiber into $\mathcal{O}\left(-\sigma_{0}\right)$. The corresponding integral transform is still a Fourier-Mukai. ${ }^{29}$ However, this special transform does not map the semistable degree zero bundles over the elliptic fiber into spectral cover. But it is in principle possible to easily study the Fourier transform of semistable sheaves over such isolated elliptic fibers. On the other hand, over generic

\footnotetext{
${ }^{29}$ The kernel of the integral transform is basically the same as the Poincare sheaf twisted by a $\mathcal{O}(+2)$.
} 
irreducible fibers, the Fourier-Mukai is just the same as the usual transform that we have used so far. So globally, the Fourier-Mukai transform of a stable degree zero bundles will be a spectral sheaf plus some additional data over the irreducible fibers. We will explain this briefly in the following.

Define the sheaf $\Sigma$ as

$$
0 \longrightarrow \Sigma \longrightarrow \mathcal{I}_{c_{1}} \otimes \mathcal{O}_{X}(2 \sigma) \longrightarrow i_{\sigma *} \mathcal{O}_{\sigma}\left(c_{1}\right) \longrightarrow 0
$$

Clearly the restriction over smooth fibers is the zero section. Let us compute the restriction over the components of the $I_{2}$ fiber

$$
L i_{c_{1}}^{*} \Sigma=\mathcal{O}_{c_{1}}(-1), \quad L i_{c_{2}}^{*} \Sigma=\mathcal{O}_{c_{2}} .
$$

So the restriction of $\Sigma$ on the reducible fiber is $\mathcal{O}\left(-\sigma_{0}\right)$

$$
0 \longrightarrow \Sigma \longrightarrow \mathcal{O}_{c_{1}}(-1) \oplus \mathcal{O}_{c_{2}} \longrightarrow T \longrightarrow 0
$$

Then define the modified Poincare sheaf as

$$
\overline{\mathcal{P}}:=\mathcal{I}_{\Delta} \otimes \pi_{1}^{*} \Sigma \otimes \pi_{2}^{*} \Sigma \otimes K_{B}^{-1} .
$$

As mentioned before with this kernel the new or modified Fourier-Mukai is almost the same as the usual one for Weierstrass models, but over the reducible fibers, it maps the degree zero line bundles to rank two bundles. For example

$$
R \pi_{2 *}\left(\pi_{1}^{*} \mathcal{O}_{E_{p}}\left(q-\sigma_{0}\right)\right)=\mathcal{F},
$$

where $q$ and $\sigma_{0}$ are points in the same component $c_{1}$, and $\mathcal{F}$ is

$$
0 \longrightarrow \Sigma^{\otimes 2} \otimes \mathcal{O}_{E_{p}}\left(p-\sigma_{0}\right) \longrightarrow \mathcal{F} \rightarrow \Sigma \longrightarrow 0
$$

So the usual "spectral data" is modified by adding some exceptional data on non-generic fiber. More clearly, if the vector bundle over the reducible fiber is a direct sum on line bundles $\bigoplus_{i} \mathcal{O}\left(q_{i}-\sigma_{0}\right)$ then the Fourier-Mukai transform of that is $\bigoplus_{i} \mathcal{F}_{i}$. We ignore the calculations, but it is basically similar to the standard case that can be found in the literature.

It is now possible to continue the classification of the "spectral data" for nonWeierstrass Calabi-Yau threefolds. However this is a very formidable work to do. Because there are hundreds of millions of Calabi-Yau threefolds and each one may have many rational zero sections that wrap around many rational curves. In addition the fibers don't necessarily degenerate in a simple form like the $I_{2}$ that we considered here, and for each case maybe another "zero section" $\Sigma$ needs to be defined. On the other hand, the F-theory dual of such heterotic models (it they exist) is not very well known at least to the author. So we leave the complete classification of the Yukawa couplings to the future. 


\section{Conclusion}

In this paper, we tried to introduce a new approach for computing the Yukawa couplings of the effective $4 D \mathcal{N}=1$ GUT theories, derived from heterotic string compactifications over Calabi-Yau threefold manifolds. The key inside was that the Fourier-Mukai transform (with the kernel being the Poincare sheaf) interchanges the tensor product and the Pontrjagin product, at least over a smooth elliptic curve. We argued that for computing the cohomologies of a product of vector bundles, we can still use the Pontjagin product in most cases. In other words, when the spectral cover has no vertical component, it is still possible to use the antisymmetrized Pontrjagin product for computing the cohomologies of $\Lambda^{2} V$. On the other hand, when there are vertical components, we used indirect computations, even though it seems it is still possible to directly use the spectral data.

Assuming the Calabi-Yau manifold is defined by the Weierstrass equation, one can classify possible spectral data that can appear for a smooth degree zero vector bundle and use the Pontrjagin product to find the relevant "pieces" of the spectral data that can give specific Yukawa couplings. This has two advantages. First, relative to the standard approaches, the calculations become much simpler, or at least the problem dissects into smaller and easy steps. Second, the relation between the intersecting brane models and Tbrane becomes very clear. We didn't study the F-theory implications of these calculations thoroughly. However, in principle, these spectral data can be used to define the Higgs bundle one the 7-branes. Hence, we can see whether the specific couplings in the effective heterotic theory result from the triple intersection of branes or the interaction of the zero modes localized inside the 7-brane and curves.

Finally, since most of the elliptically Calabi-Yau manifolds constructed so far has only rational sections, it is interesting to see whether this idea can be extended to the CalabiYau manifolds without holomorphic sections. We gave the idea of how this can be done, but the full classification of the spectral data over such manifolds is much harder, plus there are many manifolds that need to be checked. In addition, the F-theory dual of such heterotic models is not very well known. So this generalization is outside of the scope of this paper, and we leave it for the future.

\section{Acknowledgments}

MK is grateful for the insightful discussions with Tony Pantev, James Gray, Juntao Wang, Wei Cui, Seung-Joo Lee, Xin Gao, and Paul Oehlmann. Also, special thanks to Elham Mahmoudian for being supportive during the writing and preparing this paper. This work was supported by IBS under the project code, IBS-R018-D1.

\section{A Useful formulas/terminologies}

In this appendix we briefly explain the terminologies, and some of the formulas that we used in the bulk of this paper.

- Integral Transform 
Integral transform is defined as

$$
\begin{aligned}
\Phi: D(X) & \longrightarrow D(Y) \\
\Phi(\bullet) & :=R \pi_{2 *}\left(\pi_{1}^{*} \bullet \otimes K^{\bullet}\right),
\end{aligned}
$$

where $K^{\bullet}$ is an object of $D(X \times Y)$, and $\pi_{1}$ and $\pi_{2}$ are projection morphisms

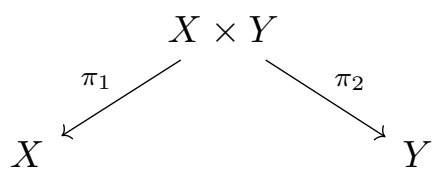

An integral transform is called a Fourier-Mukai when it is an equivalence of the derived categories. For this to be true, the kernel of the integral transform should satisfy an "orthogonality condition", see [82] Theorem $\mathbf{1 . 2 7}$

- WIT-i

As mentioned before $\Phi$ "maps" objects $D(X)$, i.e. complexes of coherent sheaves in $X$, to objects of $D(Y)$. So one can view a coherent sheaf $\mathcal{F}$ as an object in derived category

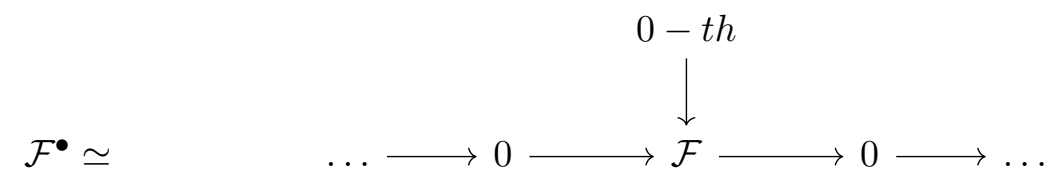

We call a sheaf $\mathcal{F}$ is WIT-i respect to the functor $\Phi$ if its integral transform $\mathcal{G}^{\bullet}=\Phi(\mathcal{F})$ is a coherent sheaf located on the i-th position

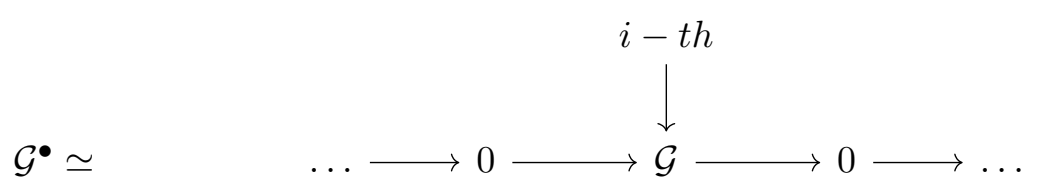

In this case we write

$$
\Phi(\mathcal{F})=\mathcal{G}[-i]
$$

Note that $[-i]$ is a shift functor, and it simply shift the complex i places to the right.

- If $V$ is a rank $n$ stable degree zero vector bundle over $\mathrm{X}$ (which is an elliptically fibered Calabi-Yau threefold), then $c_{1}\left(\pi_{*} \mathcal{L}_{n}\right)=n c_{1}\left(K_{B}\right)$.

This can be proved easily. First note that the Chern character of $\Phi(V)=i_{S_{n} *} \mathcal{L}_{n}[-1]$ can be computed in terms of the Chern classes of $V$ [80]

$$
\operatorname{ch}\left(i_{S_{n} *} \mathcal{L}_{n}\right)=\left[S_{n}\right]+\left[S_{n}\right] \cdot\left(-\frac{c_{1}(B)}{2}\right)+\frac{1}{2} c_{2}(V) f+\ldots
$$

where $\left[S_{n}\right]=n \sigma+\eta$ is the divisor class of the spectral cover, and $f$ is the class of the elliptic fiber. One can use this information, and the Grothendieck-Riemann-Roch theorem to find $\operatorname{ch}\left(\pi_{*} i_{S_{n} *} \mathcal{L}_{n}\right)$

$$
\operatorname{ch}\left(\pi_{*} i_{S_{n} *} \mathcal{L}_{n}\right)=\pi_{*}\left(\operatorname{ch}\left(i_{S_{n} *} \mathcal{L}_{n}\right) \frac{t d(X)}{t d(B)}\right)=\pi_{*}\left((n \sigma+\eta)-c_{1}(B)(n \sigma+\eta)-\frac{c_{3}(V)}{2} f+\ldots\right) .
$$


Then we use the fact that $\pi_{*} \sigma=1, \pi_{*} \eta=0$ (when we right $\eta$ we really mean $\pi^{*} \eta$ ) and $\pi_{*} f=0$. Therefore

$$
\operatorname{ch}\left(\pi_{*} i_{S_{n} *} \mathcal{L}_{n}\right)=n-n c_{1}(B)+\cdots=n+n c_{1}\left(K_{B}\right)+\ldots
$$

- Numeric Rank. By numeric rank of a sheaf $\mathcal{E}$ which is supported over a non-reduced subscheme we mean the Hilbert polynomial of that sheaf $P(s): \chi(X, \mathcal{E} \otimes \mathcal{O}(s))$, is the same as a the Hilbert polynomial of a coherent sheaf over a smooth subscheme with the same class.

- Proof of (4.10)

Consider a short exact sequence,

$$
0 \longrightarrow A \stackrel{f}{\longrightarrow} B \longrightarrow C \longrightarrow 0,
$$

where $A$ and $B$ are locally free, but $C$ can be a general coherent sheaf. In derived category we can rewrite this as an isomorphism

$$
\begin{gathered}
C^{\bullet} \quad: \cdots \longrightarrow 0 \longrightarrow C \longrightarrow 0 \longrightarrow \ldots \\
\text { Cone }(f): \cdots \longrightarrow 0 \longrightarrow A \longrightarrow B \longrightarrow 0 \\
C^{\bullet} \simeq \text { Cone }(f) .
\end{gathered}
$$

Note that $\operatorname{Cone}(f)^{0}=B$ and $\operatorname{Cone}(f)^{-1}=A$ So there is a isomorphism after antisymmetrized product

$$
\Lambda^{2} C^{\bullet} \simeq \Lambda^{2} \text { Cone }(f)
$$

When $C$ is a vector bundle

$$
\begin{aligned}
\Lambda^{2} C^{\bullet} & =\Lambda^{2} C, \\
\Lambda^{2} \text { Cone }(f) & : 0 \longrightarrow S^{2} A \longrightarrow A \otimes B \longrightarrow \Lambda^{2} B \longrightarrow 0 .
\end{aligned}
$$

Again please remember $\Lambda^{2}$ Cone $(f)^{0}=\Lambda^{2} B, \Lambda^{2}$ Cone $(f)^{-1}=A \otimes B$ and $\Lambda^{2}$ Cone $(f)^{-2}=$ $S^{2} A$. So (A.14) implies the usual long exact sequence

$$
0 \longrightarrow S^{2} A \longrightarrow A \otimes B \longrightarrow \Lambda^{2} B \longrightarrow \Lambda^{2} C \longrightarrow 0 .
$$

However, if $C$ is a torsion sheaf supported on a smooth divisor $D$

$$
C^{\bullet}=i_{D *} \mathcal{M}
$$

where $\mathcal{M}$ is line bundle on $D$, then the antidymmetrized product will be in the (-1)-th position

$$
\begin{aligned}
\Lambda^{2} C^{\bullet}=i_{D *} \mathcal{M} i_{D *} \mathcal{M} & =i_{D *}\left(\mathcal{M} \otimes_{\Lambda}\left(\mathcal{M} \oplus \mathcal{M} \otimes \mathcal{N}_{D / X}^{*}[+1]\right)\right) \\
& =i_{D *} \mathcal{M}^{\otimes 2} \otimes \mathcal{N}_{D / X}^{*}[+1]
\end{aligned}
$$


$\mathcal{N}_{D / X}$ is the normal bundle of $D$. So the isomorphism (A.14) implies

$$
\mathcal{H}^{-1}(\text { Cone }(f)) \simeq i_{D *} \mathcal{M}^{\otimes 2} \otimes \mathcal{N}_{D / X}^{*}
$$

Namely, the complex Cone $(f)$ is not exact on the middle term anymore. Using the following short exact sequence for the bundle $A$

$$
0 \longrightarrow S^{2} A \longrightarrow A \otimes A \longrightarrow \Lambda^{2} A \longrightarrow 0
$$

we can rewrite (A.20) as

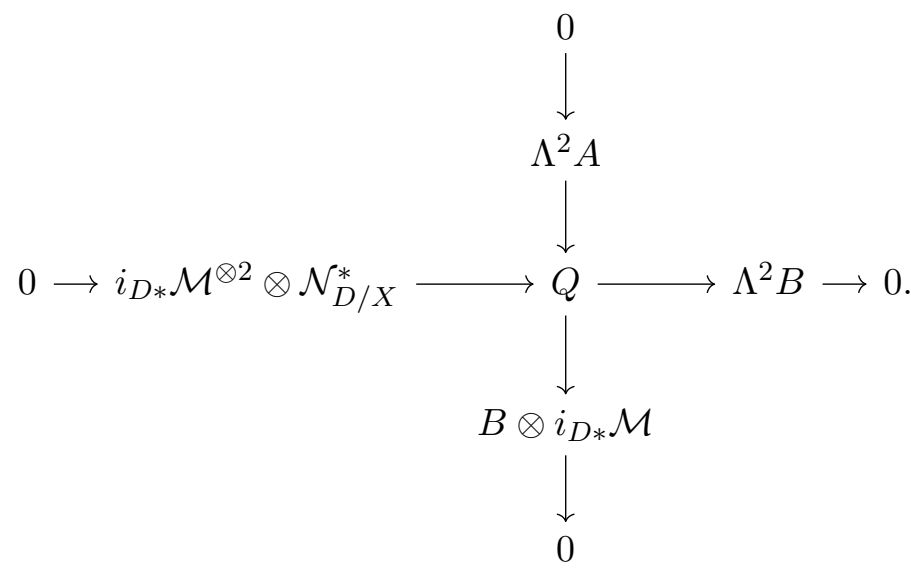

- $L i_{\sigma}^{*} \mathcal{O}_{S_{2}}(\sigma) \star \mathcal{O}_{S_{2}}(\sigma)$

Here instead of computing the Pontrjagin product we use the converse transform and compute the tensor product of the corresponding bundles and then we take the FourierMukai transform of that. First to fix the notation, assume $\left[S_{2}\right]=2 \sigma+\eta$.

It is easy to find the inverse transform of $\mathcal{O}_{S_{2}}(\sigma)$, which is denoted by $V$

$$
0 \longrightarrow \mathcal{O}_{X}(-\sigma) \longrightarrow V \longrightarrow \mathcal{O}_{X}\left(\sigma-\eta-K_{B}\right) \longrightarrow 0
$$

Now all we need is the Fourier transform of $V \otimes V$ and use the identity

$$
L i_{\sigma}^{*} \Phi^{1}(V \otimes V)=L i_{\sigma}^{*} \mathcal{O}_{S_{2}}(\sigma) \star \mathcal{O}_{S_{2}}(\sigma) \otimes K_{B}^{-1}[-1] .
$$

We ignore the detailed calculations,${ }^{30}$ and we just write the final result for $\Phi(V \otimes V)$

$$
0 \longrightarrow \mathcal{F}_{2} \longrightarrow \mathcal{F}_{1} \longrightarrow \Phi^{1}(V \otimes V) \longrightarrow 0,
$$

\footnotetext{
${ }^{30}$ All we need are the Fourier transforms of $\mathcal{O}_{X}, \mathcal{O}_{X}(2 \sigma)$ and $\mathcal{O}_{X}(-2 s)$. They can be computed by using the defining short exact sequence for $\mathcal{I}_{\Delta}[80,82]$.
} 
where $\mathcal{F}_{1}$ is defined as

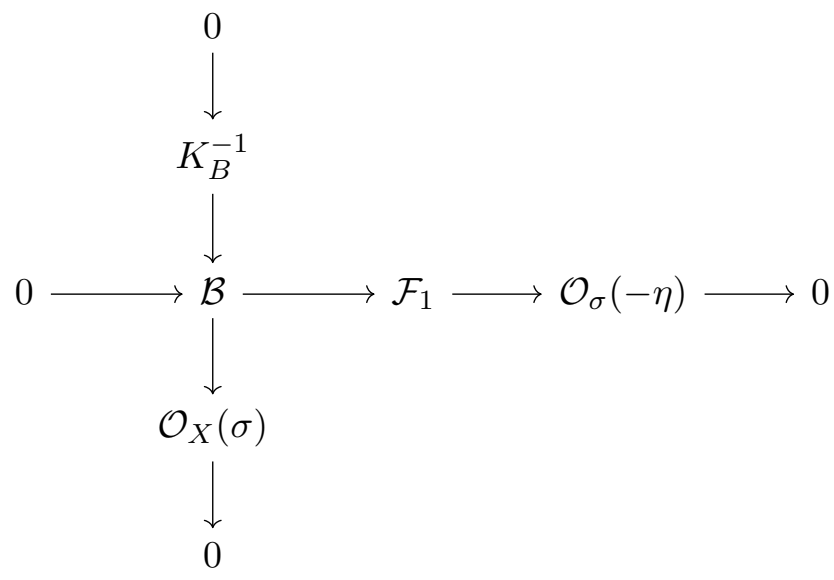

and $\mathcal{F}_{2}$,

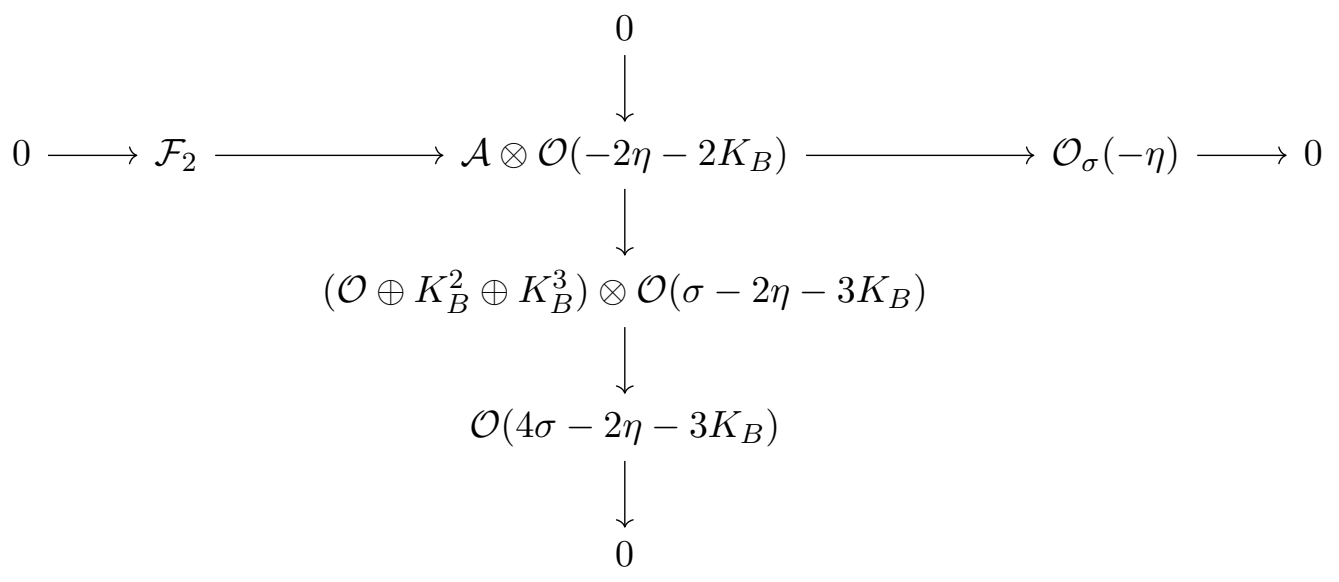

Even though these diagrams look very difficult, the restriction on the zero section is quite easy

$$
\mathcal{M}^{\bullet} \simeq \quad 0 \longrightarrow \begin{gathered}
\mathcal{O}_{B}\left(-K_{B}-\eta\right) \\
\oplus \\
\mathcal{O}_{B}\left(-3 \eta-2 K_{B}\right)
\end{gathered} \longrightarrow \begin{gathered}
\mathcal{O}_{B}\left(\eta+K_{B}\right) \\
\oplus \\
\mathcal{O}_{B}(-\eta)
\end{gathered} \longrightarrow 0
$$

where the sequence $\mathcal{M}^{\bullet}$ on the left is not exact, and it is isomorphic (in derived category $D(B))$ to the pullback $L i_{\sigma}^{*} \Phi^{1}(V \otimes V)$. This mean the zeroth cohomology $\mathcal{H}^{0}\left(\mathcal{M}^{\bullet}\right)$ of this sequence gives $i_{\sigma}^{*} \Phi^{1}(V \otimes V)$, and the "(-1)-th" cohomology $\mathcal{H}^{-1}\left(\mathcal{M}^{\bullet}\right)$ gives $L^{-1} i_{\sigma}^{*} \Phi^{1}(V \otimes V)$. In addition to this, there is a surjection

$$
V \otimes V \longrightarrow \Lambda^{2} V=\mathcal{O}_{X}\left(-\eta-K_{B}\right) \longleftrightarrow 0,
$$

which induces a surjection

$$
\mathcal{O}_{B}\left(\eta+K_{B}\right) \longrightarrow i_{\sigma}^{*} \Phi^{1}(V \otimes V) \longrightarrow \mathcal{O}_{\sigma}(-\eta) \longrightarrow 0 .
$$


On the other hand, the map between the line bundles $\mathcal{O}_{V}\left(-\eta-K_{B}\right)$ and $\mathcal{O}_{V}\left(\eta+K_{B}\right)$ can be induced by the extension morphism of the original sequence. ${ }^{31}$ They could be $a_{0} a_{2}$. But between the line bundles $\mathcal{O}_{V}\left(-3 \eta-2 K_{B}\right)$ and $\mathcal{O}_{V}\left(\eta+K_{B}\right)$ cannot be induced by the extension morphism. Since the bundle $V \otimes V$ is associated with $V$, we expect that the morphisms between the constituents of $V \otimes V$ are also induced by the morphisms that defined $V$, i.e., the extension morphism. So We conclude that $\mathcal{O}\left(-3 \eta-2 K_{B}\right)$ should be injected into $\mathcal{H}^{-1}$. But whether $\mathcal{O}\left(-\eta-K_{B}\right)$ is inside $\mathcal{H}^{-1}$ and $\mathcal{O}\left(\eta+K_{B}\right)$ is inside $\mathcal{H}^{0}$ depends on the morphism mentioned above. Therefore the final result is

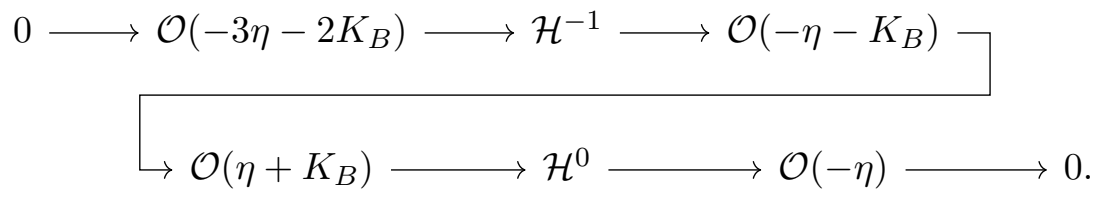

Using the relations mentioned above, we can see that this is in complete agreement with our heuristic result in $\mathrm{SU}(5)$ example.

- Using Pontrjagin Product For Double Cover With A Vertical Component.

Without loss of generality,

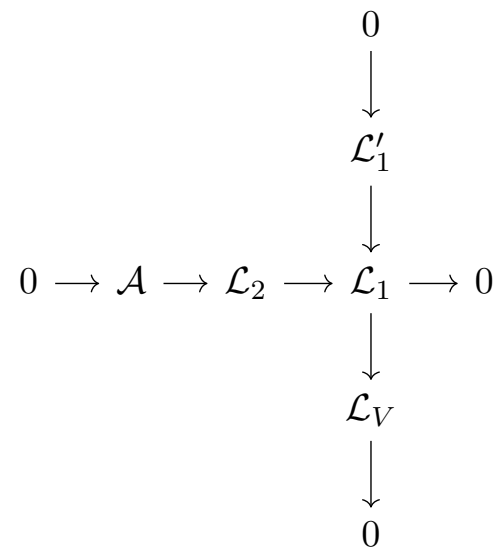

where $\mathcal{A}$ and $\mathcal{L}_{1}^{\prime}$ are line bundles supported over $\sigma$ and $\mathcal{L}_{V}$ is a line bundle over $V$. The parameters needed to define $\mathcal{L}$ are given by the extension groups,

$$
\begin{aligned}
R^{*} \operatorname{Hom}_{D(X)}\left(\mathcal{L}_{1}, \mathcal{A}\right) & =R^{*} \operatorname{Hom}_{D(B)}\left(\operatorname{Li}_{\sigma}^{*} \mathcal{L}_{1}, \mathcal{A}\right)=R^{*} \operatorname{Hom}_{D(B)}\left(\left.\mathcal{L}_{1}^{\prime} \otimes K_{B}^{-1}[+1] \oplus \mathcal{L}_{1}\right|_{\sigma}, \mathcal{A}\right) \\
R^{*} \operatorname{Hom}_{D(X)}\left(\mathcal{L}_{V}, \mathcal{L}_{1}^{\prime}\right) & =R^{*} \operatorname{Hom}_{D(c)}\left(\left.\mathcal{L}_{V}\right|_{c},\left.\mathcal{L}_{1}^{\prime}\right|_{c} \otimes \mathcal{O}_{c}(c)[-1]\right)
\end{aligned}
$$

where $c$ in the last line is the intersection of the vertical and horizontal components, and this extension group parameterizes the gluing. There are three parameters in $\operatorname{Ext}^{1}\left(\mathcal{L}_{1}, \mathcal{A}\right)$,

$$
\begin{aligned}
\mathcal{E} x t \in & \operatorname{Hom}_{D(B)}\left(\mathcal{L}_{1}^{\prime} \otimes K_{B}^{-1}, \mathcal{A}\right), \\
& \times \operatorname{Ext}_{D(B)}^{1}\left(\mathcal{L}_{1}^{\prime}, \mathcal{A}\right), \\
\operatorname{Ext}_{D(B)}^{1}\left(i_{\sigma}^{*} \mathcal{L}_{V}, \mathcal{A}\right)= & \operatorname{Hom}_{D(c)}\left(\mathcal{L}_{V}, \mathcal{A} \otimes \mathcal{O}_{c}(c)\right) .
\end{aligned}
$$

\footnotetext{
${ }^{31}$ The extension group decomposes into two subgroups $\operatorname{Ext} t^{1}\left(\mathcal{O}\left(\sigma-\eta-K_{B}\right), \mathcal{O}(-\sigma)\right)=H^{0}(\mathcal{O}(\eta)) \oplus$ $H^{0}\left(\mathcal{O}\left(\eta+2 K_{B}\right)\right)$. The choice of the extension morphism uniquely fixes the algebraic equation of the double cover $S_{2}=a_{2} X+a_{0} Z^{2}$ and the coefficients are identified with the elements of the subgroups mentioned.
} 
The first group parameterizes a line bundle over the non-reduced components. The second one gives a rank two bundle over $\sigma$, and the third group corresponds to gluing on the intersection. We turn the second factor off, so we don't get the a vector bundle over $B$. Also for finding $R \pi_{*} V$ we need to restricted the spectral data on the zero section,

$$
\begin{gathered}
0 \longrightarrow \mathcal{A} \otimes K_{B}^{-1} \longrightarrow L^{-1} i_{\sigma}^{*} \mathcal{L}_{2} \longrightarrow \mathcal{L}_{1}^{\prime} \otimes K_{B}^{-1} \\
\left.\longrightarrow \mathcal{A} \longrightarrow L^{0} i_{\sigma}^{*} \mathcal{L}_{2} \longrightarrow \mathcal{L}_{1}\right|_{\sigma} \longrightarrow 0 \\
R \pi_{*} V=\mathcal{A} \otimes K_{B}^{-1} \oplus \mathcal{R}[-1], \\
\left.\left.0 \longrightarrow \mathcal{A}\right|_{\mathcal{E} x t=0} \longrightarrow \mathcal{R} 2 \longrightarrow \mathcal{L}_{1}\right|_{\sigma} \longrightarrow 0 .
\end{gathered}
$$

The Extension for this sequence is induced by $\operatorname{Ext}_{D(B)}^{1}\left(\mathcal{L}_{1}^{\prime}, \mathcal{A}\right)$ restricted on $\mathcal{E} x t=0$. But we chose this to be zero everywhere. Also note that there is a topological constraint on this as before,

$$
c_{1}(\mathcal{A})+c_{1}\left(\mathcal{L}_{1}^{\prime}\right)+[V]=2 c_{1}\left(K_{B}\right) .
$$

There is also a smoothness condition, that is $\pi_{*} V \sim i_{\sigma}^{*} \mathcal{L}_{2}$ must be locally free (smooth coherent sheaf). Also from the reasons mentioned before $i_{V}^{*} \mathcal{L}_{2}$ must have relative +1 . Putting these requirements together one gets $\mathcal{L}_{V}$,

$$
\mathcal{L}_{V}=\mathcal{O}_{V}\left(\sigma+K_{B}-c_{1}(\mathcal{A})\right)
$$

Now we can proceed to find the Fourier transform of $\Lambda^{2} V$. To compute $\mathcal{L}_{2} \star_{A} \mathcal{L}_{2}$ we should be careful. This is because of the existence of the vertical components, which is already mentioned. To see how to deal with this, first note that,

$$
\mathcal{L}_{V} \star_{A} \mathcal{L}_{V} \simeq_{Q i s} 0 \longrightarrow \mathcal{L}_{1}^{\prime} \star_{S} \mathcal{L}_{1}^{\prime} \longrightarrow \mathcal{L}_{1}^{\prime} \star \mathcal{L}_{1} \longrightarrow \mathcal{L}_{1} \star_{A} \mathcal{L}_{1} \longrightarrow 0
$$

Instead of computing $\mathcal{L}_{V} \star_{A} \mathcal{L}_{V}$ directly, we can compute the antisymmetrized product of the inverse Fourier transform. In other words, we use,

$$
\mathcal{L}_{V \star}{ }_{A} \mathcal{L}_{V}=i_{V *} \mathcal{L}_{V} \star \mathcal{L}_{V} \otimes \mathcal{O}(-V)[+1]=i_{V *} \Phi\left(\mathcal{O}_{V}\right)\left(-2 \sigma+c_{1}\left(\mathcal{L}_{1}^{\prime}\right)-c_{1}(\mathcal{A})\right) \otimes K_{B}[+2] .
$$

It is easy to compute the right hand side,

$$
\begin{aligned}
& 0 \longrightarrow K_{B}^{-1} \longrightarrow \mathcal{G} \longrightarrow \mathcal{O}(\sigma) \longrightarrow 0 \\
& \mathcal{L}_{V} \star_{A} \mathcal{L}_{V}=i_{V *} \mathcal{G} \otimes \mathcal{O}\left(+K_{B}+c_{1}\left(\mathcal{L}_{1}^{\prime}\right)-c_{1}(\mathcal{A})\right)[+1]
\end{aligned}
$$


Therefore one gets a diagram as follows,

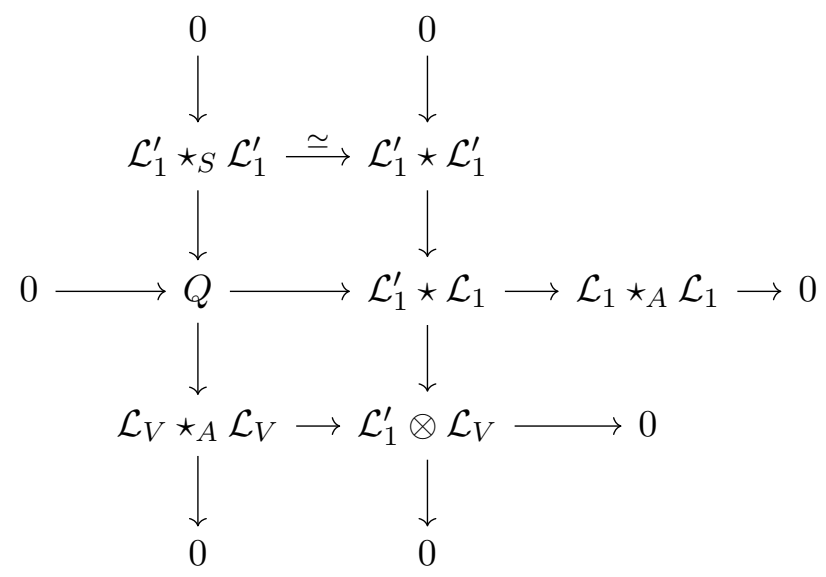

Putting every thing together we get the result for $\mathcal{L}_{1} \star_{A} \mathcal{L}_{1}$,

$$
\mathcal{L}_{1} \star_{A} \mathcal{L}_{1}=\mathcal{O}_{V}\left(c_{1}\left(\mathcal{L}_{1}^{\prime}\right)-c_{1}(\mathcal{A})\right)[+1]
$$

The idea that we want to emphasize is that we can continue this approach for finding the "antisymmetric" Pontrjagin product of spectral sheaves of higher degrees. So when we add another sheet, one gets the following quasi-isomorphism,

$$
\begin{aligned}
& \mathcal{L}_{1} \star_{A} \mathcal{L}_{1} \simeq_{Q i s} \\
& 0 \longrightarrow \mathcal{A} \star_{S} \mathcal{A} \longrightarrow \pi^{*} \pi_{*} \mathcal{A} \otimes \mathcal{L}_{2} \longrightarrow \mathcal{L}_{2} \star_{A} \mathcal{L}_{2} \longrightarrow 0 .
\end{aligned}
$$

Similar to the previous part, this quasi isomorphism is equivalent to the following diagram,

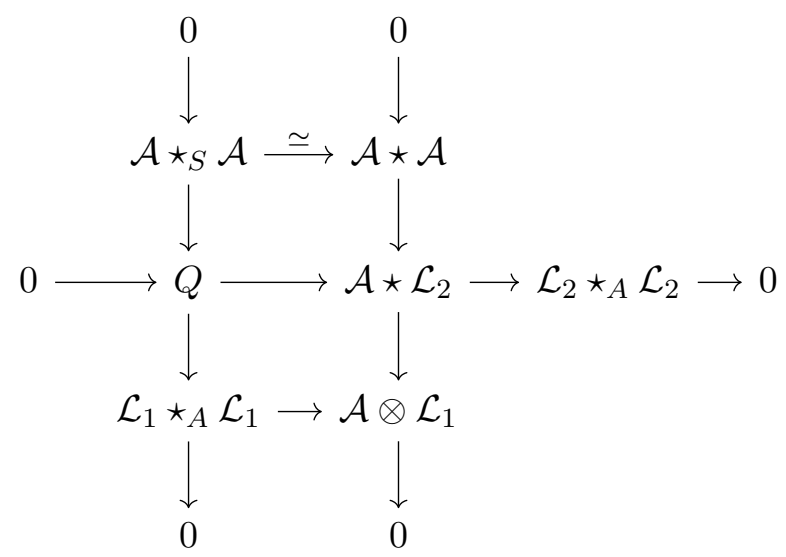

Again by putting the pieces together and chasing the diagrams, one gets the final result, (if $c_{1}(\mathcal{A})-c_{1}\left(\mathcal{L}_{1}^{\prime}+c_{1}\left(K_{B}\right)\right)=0$, which is induced again by the stability of $V_{2}$ ) one gets,

$$
\mathcal{L}_{2} \star_{A} \mathcal{L}_{2}=\mathcal{O}_{\sigma} \otimes K_{B}^{\otimes 2}
$$

This consistent with the expectation of $\Lambda^{2} V=\mathcal{O}_{X}$. 
Open Access. This article is distributed under the terms of the Creative Commons Attribution License (CC-BY 4.0), which permits any use, distribution and reproduction in any medium, provided the original author(s) and source are credited.

\section{References}

[1] P. Candelas, G.T. Horowitz, A. Strominger and E. Witten, Vacuum configurations for superstrings, Nucl. Phys. B 258 (1985) 46 [INSPIRE].

[2] P. Green and T. Hubsch, Calabi-Yau manifolds as complete intersections in products of complex projective spaces, Commun. Math. Phys. 109 (1987) 99 [INSPIRE].

[3] J. Distler and B.R. Greene, Aspects of $(2,0)$ string compactifications, Nucl. Phys. B 304 (1988) 1 [INSPIRE].

[4] B.R. Greene, K.H. Kirklin, P.J. Miron and G.G. Ross, A three generation superstring model. 1. Compactification and discrete symmetries, Nucl. Phys. B 278 (1986) 667 [INSPIRE].

[5] B.R. Greene, K.H. Kirklin, P.J. Miron and G.G. Ross, A three generation superstring model. 2. Symmetry breaking and the low-energy theory, Nucl. Phys. B 292 (1987) 606 [INSPIRE].

[6] P. Candelas, C.A. Lütken and R. Schimmrigk, Complete intersection Calabi-Yau manifolds. 2. Three generation manifolds, Nucl. Phys. B 306 (1988) 113 [inSPIRE].

[7] S. Kachru, Some three generation (0,2) Calabi-Yau models, Phys. Lett. B 349 (1995) 76 [hep-th/9501131] [INSPIRE].

[8] V. Braun, Y.-H. He, B.A. Ovrut and T. Pantev, A standard model from the $E_{8} \times E_{8}$ heterotic superstring, JHEP 06 (2005) 039 [hep-th/0502155] [INSPIRE].

[9] V. Braun, Y.-H. He, B.A. Ovrut and T. Pantev, A heterotic standard model, Phys. Lett. B 618 (2005) 252 [hep-th/0501070] [INSPIRE].

[10] V. Braun, Y.-H. He, B.A. Ovrut and T. Pantev, The exact MSSM spectrum from string theory, JHEP 05 (2006) 043 [hep-th/0512177] [INSPIRE].

[11] V. Braun, P. Candelas, R. Davies and R. Donagi, The MSSM spectrum from (0,2)-deformations of the heterotic standard embedding, JHEP 05 (2012) 127 [arXiv: 1112.1097] [INSPIRE].

[12] V. Bouchard and R. Donagi, An SU(5) heterotic standard model, Phys. Lett. B 633 (2006) 783 [hep-th/0512149] [INSPIRE].

[13] R. Blumenhagen, S. Moster and T. Weigand, Heterotic GUT and standard model vacua from simply connected Calabi-Yau manifolds, Nucl. Phys. B 751 (2006) 186 [hep-th/0603015] [INSPIRE].

[14] L.B. Anderson, Y.-H. He and A. Lukas, Monad bundles in heterotic string compactifications, JHEP 07 (2008) 104 [arXiv: 0805.2875] [INSPIRE].

[15] Y.-H. He, S.-J. Lee and A. Lukas, Heterotic models from vector bundles on toric Calabi-Yau manifolds, JHEP 05 (2010) 071 [arXiv:0911.0865] [INSPIRE].

[16] L.B. Anderson, J. Gray, A. Lukas and E. Palti, Two hundred heterotic standard models on smooth Calabi-Yau threefolds, Phys. Rev. D 84 (2011) 106005 [arXiv:1106.4804] [INSPIRE].

[17] L.B. Anderson, J. Gray, A. Lukas and E. Palti, Heterotic line bundle standard models, JHEP 06 (2012) 113 [arXiv:1202.1757] [INSPIRE]. 
[18] L.B. Anderson, A. Constantin, J. Gray, A. Lukas and E. Palti, A comprehensive scan for heterotic SU(5) GUT models, JHEP 01 (2014) 047 [arXiv:1307.4787] [INSPIRE].

[19] Y.-H. He, S.-J. Lee, A. Lukas and C. Sun, Heterotic model building: 16 special manifolds, JHEP 06 (2014) 077 [arXiv: 1309.0223] [INSPIRE].

[20] A. Constantin, Heterotic string models on smooth Calabi-Yau threefolds, Ph.D. thesis, Oxford U., Oxford, U.K. (2018) [arXiv: 1808.09993] [INSPIRE].

[21] A. Constantin, Y.-H. He and A. Lukas, Counting string theory standard models, Phys. Lett. B 792 (2019) 258 [arXiv:1810.00444] [INSPIRE].

[22] M. Larfors, D. Passaro and R. Schneider, Heterotic line bundle models on generalized complete intersection Calabi Yau manifolds, JHEP 05 (2021) 105 [arXiv:2010.09763] [INSPIRE].

[23] L.B. Anderson, Heterotic and M-theory compactifications for string phenomenology, arXiv:0808.3621 [INSPIRE].

[24] W. Cui and J. Gray, Numerical metrics, curvature expansions and Calabi-Yau manifolds, JHEP 05 (2020) 044 [arXiv: 1912.11068] [INSPIRE].

[25] S.K. Donaldson, Some numerical results in complex differential geometry, Pure Appl. Math. Quart. 5 (2009) 571 [math.DG/0512625].

[26] M. Headrick and T. Wiseman, Numerical Ricci-flat metrics on K3, Class. Quant. Grav. 22 (2005) 4931 [hep-th/0506129] [INSPIRE].

[27] M. Headrick and A. Nassar, Energy functionals for Calabi-Yau metrics, Adv. Theor. Math. Phys. 17 (2013) 867 [arXiv:0908.2635] [INSPIRE].

[28] M.R. Douglas, R.L. Karp, S. Lukic and R. Reinbacher, Numerical solution to the hermitian Yang-Mills equation on the Fermat quintic, JHEP 12 (2007) 083 [hep-th/0606261] [INSPIRE].

[29] M.R. Douglas, S. Lakshminarasimhan and Y. Qi, Numerical Calabi-Yau metrics from holomorphic networks, arXiv:2012.04797 [INSPIRE].

[30] M.R. Douglas, R.L. Karp, S. Lukic and R. Reinbacher, Numerical Calabi-Yau metrics, J. Math. Phys. 49 (2008) 032302 [hep-th/0612075] [inSPIRE].

[31] V. Braun, T. Brelidze, M.R. Douglas and B.A. Ovrut, Calabi-Yau metrics for quotients and complete intersections, JHEP 05 (2008) 080 [arXiv:0712.3563] [INSPIRE].

[32] V. Braun, T. Brelidze, M.R. Douglas and B.A. Ovrut, Eigenvalues and eigenfunctions of the scalar Laplace operator on Calabi-Yau manifolds, JHEP 07 (2008) 120 [arXiv:0805.3689] [INSPIRE].

[33] L.B. Anderson, V. Braun, R.L. Karp and B.A. Ovrut, Numerical Hermitian Yang-Mills connections and vector bundle stability in heterotic theories, JHEP 06 (2010) 107 [arXiv: 1004 . 4399] [INSPIRE].

[34] L.B. Anderson, V. Braun and B.A. Ovrut, Numerical hermitian Yang-Mills connections and Kähler cone substructure, JHEP 01 (2012) 014 [arXiv:1103.3041] [INSPIRE].

[35] L.B. Anderson, M. Gerdes, J. Gray, S. Krippendorf, N. Raghuram and F. Ruehle, Moduli-dependent Calabi-Yau and $\mathrm{SU}(3)$-structure metrics from machine learning, JHEP 05 (2021) 013 [arXiv : 2012.04656] [inSPIRE]. 
[36] A. Ashmore, Y.-H. He and B.A. Ovrut, Machine learning Calabi-Yau metrics, Fortsch. Phys. 68 (2020) 2000068 [arXiv: 1910.08605] [INSPIRE].

[37] c. Blesneag, E.I. Buchbinder, A. Constantin, A. Lukas and E. Palti, Matter field Kähler metric in heterotic string theory from localisation, JHEP 04 (2018) 139 [arXiv: 1801.09645] [INSPIRE].

[38] R. Deen, Y.-H. He, S.-J. Lee and A. Lukas, Machine learning string standard models, arXiv:2003.13339 [INSPIRE].

[39] A. Strominger, Yukawa couplings in superstring compactification, Phys. Rev. Lett. 55 (1985) 2547 [INSPIRE].

[40] M.B. Green, J.H. Schwarz and E. Witten, Superstring theory. Volume 2: loop amplitudes, anomalies and phenomenology, Cambridge Univ. Pr., Cambridge, U.K. (1987).

[41] P. Candelas, Yukawa couplings between $(2,1)$ forms, Nucl. Phys. B 298 (1988) 458 [inSPIRE].

[42] B.R. Greene, K.H. Kirklin, P.J. Miron and G.G. Ross, $27^{3}$ Yukawa couplings for a three generation superstring model, Phys. Lett. B 192 (1987) 111 [INSPIRE].

[43] J. Distler, B.R. Greene, K. Kirklin and P. Miron, Evaluation of $27^{3}$ Yukawa couplings in a three generation superstring model, Phys. Lett. B 195 (1987) 41 [INSPIRE].

[44] L.B. Anderson, J. Gray, D. Grayson, Y.-H. He and A. Lukas, Yukawa couplings in heterotic compactification, Commun. Math. Phys. 297 (2010) 95 [arXiv:0904.2186] [InSPIRE].

[45] L.B. Anderson, J. Gray and B. Ovrut, Yukawa textures from heterotic stability walls, JHEP 05 (2010) 086 [arXiv: 1001.2317] [INSPIRE].

[46] R. Donagi, R. Reinbacher and S.-T. Yau, Yukawa couplings on quintic threefolds, hep-th/0605203 [INSPIRE].

[47] S. Blesneag, E.I. Buchbinder, P. Candelas and A. Lukas, Holomorphic Yukawa couplings in heterotic string theory, JHEP 01 (2016) 152 [arXiv: 1512.05322] [INSPIRE].

[48] S. Blesneag, E.I. Buchbinder and A. Lukas, Holomorphic Yukawa couplings for complete intersection Calabi-Yau manifolds, JHEP 01 (2017) 119 [arXiv:1607.03461] [INSPIRE].

[49] C. Beasley, J.J. Heckman and C. Vafa, GUTs and exceptional branes in F-theory - I, JHEP 01 (2009) 058 [arXiv: 0802.3391] [inSPIRE].

[50] C.-C. Chiou, A.E. Faraggi, R. Tatar and W. Walters, T-branes and Yukawa couplings, JHEP 05 (2011) 023 [arXiv: 1101.2455] [INSPIRE].

[51] L.B. Anderson, J.J. Heckman and S. Katz, T-branes and geometry, JHEP 05 (2014) 080 [arXiv:1310.1931] [INSPIRE].

[52] S. Cecotti, C. Cordova, J.J. Heckman and C. Vafa, T-branes and monodromy, JHEP 07 (2011) 030 [arXiv: 1010.5780] [INSPIRE].

[53] T. Weigand, F-theory, PoS TASI2017 (2018) 016 [arXiv:1806.01854] [INSPIRE].

[54] M. Cvetič, D. Klevers, D.K.M. Peña, P.-K. Oehlmann and J. Reuter, Three-family particle physics models from global F-theory compactifications, JHEP 08 (2015) 087 [arXiv: 1503.02068] [INSPIRE].

[55] L.B. Anderson, J. Gray, M. Larfors, M. Magill and R. Schneider, Generalized vanishing theorems for Yukawa couplings in heterotic compactifications, JHEP 05 (2021) 085 [arXiv: 2103.10454] [INSPIRE]. 
[56] E.I. Buchbinder, A. Constantin, J. Gray and A. Lukas, Yukawa unification in heterotic string theory, Phys. Rev. D 94 (2016) 046005 [arXiv: 1606.04032] [InSPIRE].

[57] L.B. Anderson, X. Gao, J. Gray and S.-J. Lee, Fibrations in CICY threefolds, JHEP 10 (2017) 077 [arXiv : 1708.07907] [inSPIRE].

[58] Y.-C. Huang and W. Taylor, On the prevalence of elliptic and genus one fibrations among toric hypersurface Calabi-Yau threefolds, JHEP 03 (2019) 014 [arXiv:1809.05160] [INSPIRE].

[59] R. Friedman, J. Morgan and E. Witten, Vector bundles and F-theory, Commun. Math. Phys. 187 (1997) 679 [hep-th/9701162] [INSPIRE].

[60] R. Friedman, J.W. Morgan and E. Witten, Vector bundles over elliptic fibrations, alg-geom/9709029 [INSPIRE].

[61] R. Donagi, Spectral covers, Math. Sci. Res. Inst. Publ. 28 (1995) 65 [alg-geom/9505009].

[62] R. Donagi, A. Lukas, B.A. Ovrut and D. Waldram, Holomorphic vector bundles and nonperturbative vacua in M-theory, JHEP 06 (1999) 034 [hep-th/9901009] [INSPIRE].

[63] R. Donagi, B.A. Ovrut, T. Pantev and D. Waldram, Standard model bundles, Adv. Theor. Math. Phys. 5 (2002) 563 [math.AG/0008010] [INSPIRE].

[64] R. Donagi, B.A. Ovrut, T. Pantev and D. Waldram, Standard model bundles on nonsimply connected Calabi-Yau threefolds, JHEP 08 (2001) 053 [hep-th/0008008] [INSPIRE].

[65] R. Donagi, Y.-H. He, B.A. Ovrut and R. Reinbacher, The particle spectrum of heterotic compactifications, JHEP 12 (2004) 054 [hep-th/0405014] [INSPIRE].

[66] R. Donagi, Y.-H. He, B.A. Ovrut and R. Reinbacher, The spectra of heterotic standard model vacua, JHEP 06 (2005) 070 [hep-th/0411156] [INSPIRE].

[67] R. Donagi, Y.-H. He, B.A. Ovrut and R. Reinbacher, Moduli dependent spectra of heterotic compactifications, Phys. Lett. B 598 (2004) 279 [hep-th/0403291] [INSPIRE].

[68] R. Blumenhagen, S. Moster, R. Reinbacher and T. Weigand, Massless spectra of three generation $\mathrm{U}(N)$ heterotic string vacua, JHEP 05 (2007) 041 [hep-th/0612039] [INSPIRE].

[69] M. Gabella, Y.-H. He and A. Lukas, An abundance of heterotic vacua, JHEP 12 (2008) 027 [arXiv:0808.2142] [INSPIRE].

[70] V. Braun, B.A. Ovrut, T. Pantev and R. Reinbacher, Elliptic Calabi-Yau threefolds with $Z_{3} \times Z_{3}$ Wilson lines, JHEP 12 (2004) 062 [hep-th/0410055] [INSPIRE].

[71] V. Braun, Y.-H. He, B.A. Ovrut and T. Pantev, Vector bundle extensions, sheaf cohomology, and the heterotic standard model, Adv. Theor. Math. Phys. 10 (2006) 525 [hep-th/0505041] [INSPIRE].

[72] A.P. Braun, C.R. Brodie and A. Lukas, Heterotic line bundle models on elliptically fibered Calabi-Yau three-folds, JHEP 04 (2018) 087 [arXiv: 1706.07688] [INSPIRE].

[73] V. Bouchard, M. Cvetič and R. Donagi, Tri-linear couplings in an heterotic minimal supersymmetric standard model, Nucl. Phys. B $\mathbf{7 4 5}$ (2006) 62 [hep-th/0602096] [INSPIRE].

[74] V. Braun, Y.-H. He and B.A. Ovrut, Yukawa couplings in heterotic standard models, JHEP 04 (2006) 019 [hep-th/0601204] [INSPIRE].

[75] V. Braun, Y.-H. He, B.A. Ovrut and T. Pantev, Moduli dependent mu-terms in a heterotic standard model, JHEP 03 (2006) 006 [hep-th/0510142] [INSPIRE]. 
[76] H. Hayashi, T. Kawano, R. Tatar and T. Watari, Codimension-3 singularities and Yukawa couplings in F-theory, Nucl. Phys. B 823 (2009) 47 [arXiv:0901.4941] [InSPIRE].

[77] H. Hayashi, R. Tatar, Y. Toda, T. Watari and M. Yamazaki, New aspects of heterotic-F theory duality, Nucl. Phys. B 806 (2009) 224 [arXiv:0805.1057] [INSPIRE].

[78] A.P. Braun and T. Watari, On singular fibres in F-theory, JHEP 07 (2013) 031 [arXiv: 1301.5814] [INSPIRE].

[79] M. Bershadsky, T.M. Chiang, B.R. Greene, A. Johansen and C.I. Lazaroiu, F theory and linear sigma models, Nucl. Phys. B 527 (1998) 531 [hep-th/9712023] [INSPIRE].

[80] L.B. Anderson, X. Gao and M. Karkheiran, Extending the geometry of heterotic spectral cover constructions, Nucl. Phys. B 956 (2020) 115003 [arXiv:1912.00971] [INSPIRE].

[81] L.B. Anderson, H. Feng, X. Gao and M. Karkheiran, Heterotic/heterotic and heterotic/F-theory duality, Phys. Rev. D 100 (2019) 126014 [arXiv:1907.04395] [InSPIRE].

[82] C. Bartocci, U. Bruzzo and D. Hernández Ruipérez, Fourier-Mukai and Nahm transforms in geometry and mathematical physics, Birkhäuser, Boston, MA, U.S.A. (2009) [INSPIRE].

[83] J. Gray and J. Wang, Jumping spectra and vanishing couplings in heterotic line bundle standard models, JHEP 11 (2019) 073 [arXiv:1906.09373] [INSPIRE].

[84] L.B. Anderson, J. Gray, A. Lukas and B. Ovrut, The edge of supersymmetry: stability walls in heterotic theory, Phys. Lett. B 677 (2009) 190 [arXiv:0903.5088] [InSPIRE].

[85] L.B. Anderson, J. Gray, A. Lukas and B. Ovrut, Stability walls in heterotic theories, JHEP 09 (2009) 026 [arXiv:0905.1748] [INSPIRE].

[86] R. Donagi and M. Wijnholt, Model building with F-theory, Adv. Theor. Math. Phys. 15 (2011) 1237 [arXiv:0802.2969] [INSPIRE].

[87] R. Donagi and M. Wijnholt, Higgs bundles and UV completion in F-theory, Commun. Math. Phys. 326 (2014) 287 [arXiv:0904.1218] [INSPIRE].

[88] R. Donagi and M. Wijnholt, Gluing branes, I, JHEP 05 (2013) 068 [arXiv:1104.2610] [INSPIRE].

[89] R. Donagi and M. Wijnholt, Breaking GUT groups in F-theory, Adv. Theor. Math. Phys. 15 (2011) 1523 [arXiv:0808.2223] [INSPIRE].

[90] R. Donagi, S. Katz and E. Sharpe, Spectra of D-branes with Higgs vevs, Adv. Theor. Math. Phys. 8 (2004) 813 [hep-th/0309270] [InSPIRE].

[91] E. Sharpe, Lectures on D-branes and sheaves, hep-th/0307245 [INSPIRE].

[92] G. Curio, Moduli restriction and chiral matter in heterotic string compactifications, JHEP 01 (2012) 015 [arXiv:1110.6315] [INSPIRE].

[93] W. Cui and M. Karkheiran, Heterotic complex structure moduli stabilization for elliptically fibered Calabi-Yau manifolds, JHEP 03 (2021) 281 [arXiv:2011.14304] [INSPIRE].

[94] L.B. Anderson, J. Gray, N. Raghuram and W. Taylor, Matter in transition, JHEP 04 (2016) 080 [arXiv: 1512.05791] [INSPIRE].

[95] R. Friedman, Algebraic surfaces and holomorphic vector bundles, Springer, New York, NY, U.S.A. (1998).

[96] M.F. Atiyah, Complex analytic connections in fibre bundles, Trans. Amer. Math. Soc. 85 (1957) 181.

[97] A. Căldăraru, Derived categories of twisted sheaves on elliptic threefolds, math.AG/0012083. 